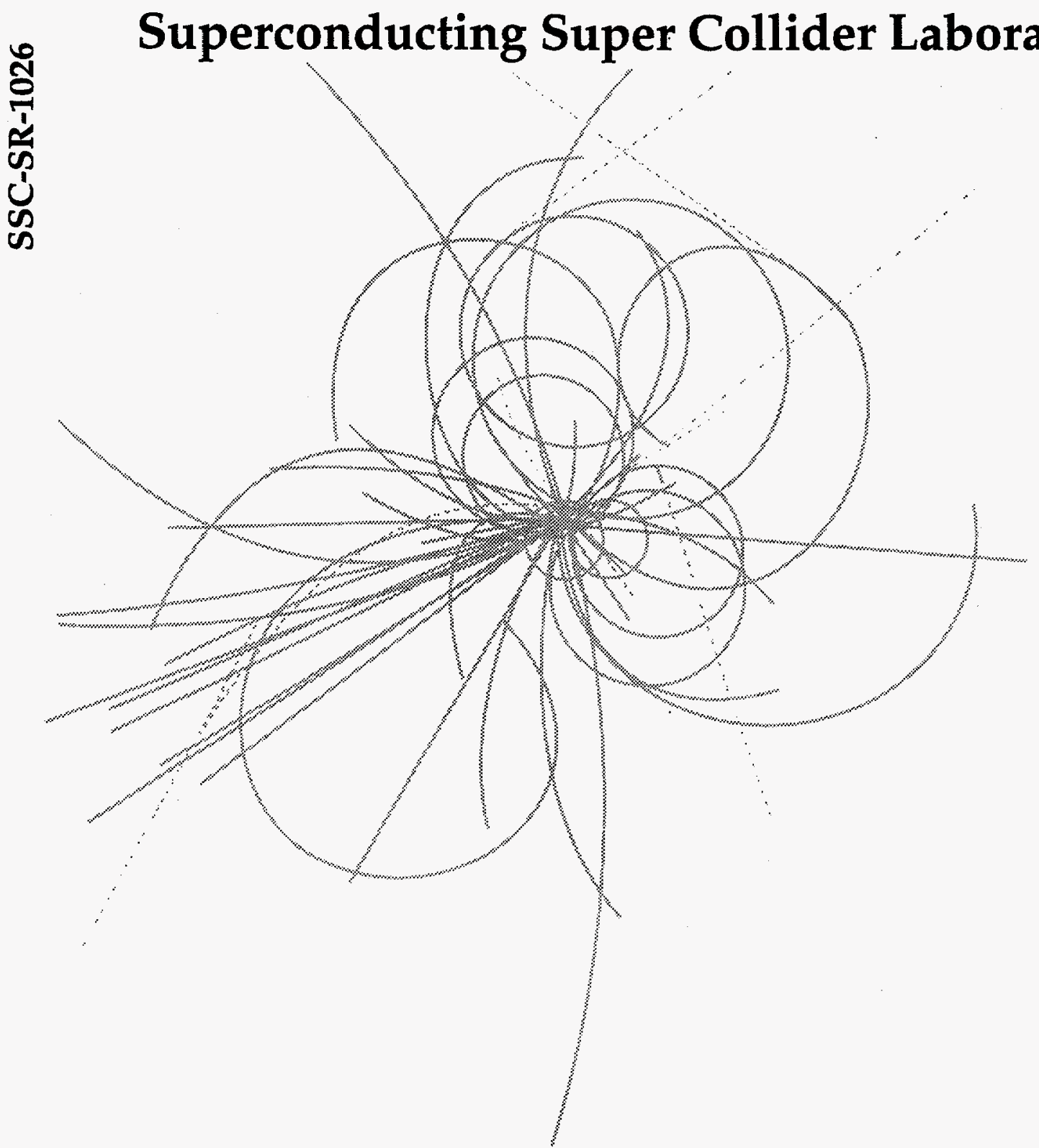

\title{
SSC Environmental Radiation Shielding
}

\author{
Task Force Report \\ J. D. Jackson, Editor
}

July 1987

APPROVED FOR RELEASE OR OATION - O.R. PATENT GROUP PUBLIGATHON - O.R. PATE 1 2.1.5.

\section{MASTER}


SSC-SR-1026

1 July 1987

\title{
SSC ENVIRONMENTAL RADIATION SHIELDING
}

\author{
Task Force Report \\ J. D. Jackson, Editor
}

\author{
SSC Central Design Group* \\ Lawrence Berkeley Laboratory 90-4040 \\ Berkeley, California 94720
}

\begin{abstract}
DISCLAIMER
This report was prepared as an account of work sponsored by an agency of the United States Government. Neither the United States Government nor any agency thereof, nor any of their employees, makes any warranty, express or implied, or assumes any legal liability or responsibility for the accuracy, completeness, or usefulness of any information, apparatus, product, or process disclosed, or represents that its use would not infringe privately owned rights. Reference herein to any specific commercial product, process, or service by trade name, trademark, manufacturer, or otherwise does not necessarily constitute or imply its endorsement, recommendation, or favoring by the United States Government or any agency thereof. The views and opinions of authors expressed herein do not necessarily state or reflect those of the United States Government or any agency thereof.
\end{abstract}

* Operated by the Universities Research Association for the Department of Energy. 


\section{DISCLAIMER}

Portions of this document may be illegible in electronic image products. Images are produced from the best available original document. 


\section{SUMMARY}

The environmental radiation shielding requirements of the SSC have been evaluated using currently available computational tools that incorporate the well known processes of energy loss and degradation of high energy particles into Monte Carlo computer codes. These tools permit determination of isodose contours in the matter surrounding a source point and therefore the specification of minimum thicknesses or extents of shielding in order to assure annual dose equivalents less than some specified design amount. For the general public the annual dose equivalent specified in the design is 10 millirem, small compared to the dose from naturally occurring radiation.

The types of radiation fall into two classes for the purposes of shielding determinations-hadrons and muons. The sources of radiation at the SSC of concern for the surrounding environment are the interaction regions, the specially designed beam dumps into which the beams are dumped from time to time, and beam clean-up regions where stops remove the beam halo in order to reduce experimental backgrounds. A final, unlikely source of radiation considered is the accidental loss of the full beam at some point around the ring. Conservative choices of a luminosity of $10^{34} \mathrm{~cm}^{-2} \mathrm{~s}^{-1}$ (ten times the design value) and a beam current three times design (corresponding to $4 \times 10^{14}$ protons in each circulating beam) have been made in calculating the required shielding and boundaries of the facility. In addition to determination of minimum distances for the annual dose equivalents, the question of possible radioactivity produced in nearby wells or in municipal water supplies is addressed. The designed shielding distances and beam dumps are such that the induced radioactivity in ground water is safely smaller than the levels permitted by EPA and international agencies.

A primary quantity resulting from this study is the recommended minimum radius of 30 feet of light earth or similar shielding material outside of the 10 -foot diameter tunnel of the SSC. (The thickness may be less if the soil is denser.) Another important distance in the plane of the accelerator is the recommended secondary (muon) shielding, outward from the tunnel all around the ring. Near the interaction regions and the beam dumps longer regions of land must be reserved for muon shielding. The behavior of the muons is such that the land above these regions may have shared use provided the plane of the tunnel is 50 feet or more below the surface.

The conclusion of the report is that, with appropriate land acquisition in the neighborhood of the technical parts of the accelerator, the facility can be operated in a safe manner with respect to environmental radiation exposure to the general public. 


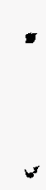




\section{TABLE OF CONTENTS}

Summary . . . . . . . . . . . . . . . . iii

Table of Contents . . . . . . . . . . . . . . . . . . v

List of Figures . . . . . . . . . . . . . . . . . . . . . vii

1. Introduction . . . . . . . . . . . . . . . 1

1.1 Sources of Radiation . . . . . . . . . . . . . . . . . . . . 4

1.2 Types of Radiation . . . . . . . . . . . . . . . . . . . . 6

1.3 Further Discussion of Sources and Shielding . . . . . . . . . . 8

1.4 Preview of the Report . . . . . . . . . . . . . . . . . . . 11

2. Computational Tools . . . . . . . . . . . . . . . . . . 13

2.1 Available Cascade Simulation Codes . . . . . . . . . . . . . 14

2.2 Particle Production Codes . . . . . . . . . . . . . . . . . 16

2.3 Muons . . . . . . . . . . . . . . . . . . . . . . . . . 17

2.4 Definitions of Absorbed Dose and Dose Equivalent . . . . . . . 21

2.5 Conversion of Simulation Results to Radiological Units . . . . . 22

2.6 Comparisons of the Tools . . . . . . . . . . . . . . . . . . 23

3. Results . . . . . . . . . . . . . . . . . . . 25

3.1 Annual Dose Equivalent from Hadrons-Beam Dump . . . . . 25

3.2 Annual Dose Equivalent from Hadrons-Interaction Regions . . 26

3.3 Annual Dose Equivalent from Hadrons-Beam Cleanup Regions . 31

3.4 Dose Equivalent from Hadrons-Accidental Beam Loss . . . . . 31

3.5 Annual Dose Equivalent from Muons-Beam Dump . . . . . . 36

3.6 Annual Dose Equivalent from Muons-Interaction Regions . . . 36

3.7 Annual Dose Equivalent from Muons-Beam Cleanup Regions . 41

3.8 Dose Equivalent from Muons-Accidental Beam Loss . . . . . . 48

3.9 Comments on the Accuracy of the Calculations . . . . . . . . 48

3.10 Radiological Consideration for Ground Water . . . . . . . . 50

4. Analysis . . . . . . . . . . . . . . . . . . 61

5. Conclusions . . . . . . . . . . . . . . . . . . . 69

Acknowledgements . . . . . . . . . . . . . . . . . 71 
Appendix A: Membership of the Task Force, Consultants, and Charge . 73

Appendix B: Comparisons among Calculations and with Data . . . 75

Appendix C: Radioactivity Induced in Nearby Well by

Accidental Loss of Beam . . . . . . . . . . . . . . 87

References . . . . . . . . . . . . . . . . . 93 


\section{LIST OF FIGURES}

Fig. 1. Schematic plan view of SSC . . . . . . . . . . . . . . . 2

Fig. 2. SSC tunnel cross section . . . . . . . . . . . . . . . . . . . 3

Fig. 3. Muon flux-different contributions . . . . . . . . . . . . . 19

Fig. 4. Prompt muon energy spectra at $40 \mathrm{TeV}$. . . . . . . . . . . . 20

Fig. 5. Isodose contours-hadrons, beam dump . . . . . . . . . . . . . 27

Fig. 6. Annual dose equivalent versus radius-hadrons, beam dump . . . . 28

Fig. 7. Isodose contours-hadrons, interaction region . . . . . . . . . . 29

Fig. 8. Annual dose equivalent versus radius-hadrons, interaction region . 30

Fig. 9. Isodose contours-hadrons, beam scrapers 1 . . . . . . . . . . . 32

Fig. 10. Isodose contours-hadrons, beam scrapers 2 . . . . . . . . . . . 33

Fig. 11. Isodose contours-hadrons, beam scrapers 3 . . . . . . . . . . 34

Fig. 12. Dose equivalent versus radius-hadrons, beam scrapers . . . . . . 35

Fig. 13. Isodose contours-muons, beam dump . . . . . . . . . . . . . 37

Fig. 14. Annual dose equivalent vs. tangential length-muons, beam dump . 38

Fig. 15. Isodose contours-muons, interaction region, far away . . . . . . . 39

Fig. 16. Annual dose equivalent versus tangential distance-muons, interaction region, far away . . . . . . . . . . . . . 40

Fig. 17. Isodose contours-muons, interaction region, close in . . . . . . . 42

Fig. 18. Annual dose equivalent versus radius-muons, IR side wall . . . . 43

Fig. 19. Isodose contours-muons, beam scrapers 1 . . . . . . . . . . . 44

Fig. 20. Isodose contours-muons, beam scrapers 2 . . . . . . . . . . . 45

Fig. 21. Isodose contours-muons, beam scrapers 3 . . . . . . . . . . . 46

Fig. 22. Dose equivalent versus tangential distance-muons, beam scrapers . 47

Fig. 23. Longitudinally integrated star production per proton versus radius in soil . . . . . . . . . . . . . . . . . . . 52

Fig. 24. Peak specific activity of ${ }^{3} \mathrm{H}$ and ${ }^{22} \mathrm{Na}$ in well water versus distance . 54

Fig. 25. Schematic diagram of SSC beam dump . . . . . . . . . . . . . 56

Fig. 26. Radially integrated star production per proton versus longitudinal distance in carbon dump . . . . . . . . . . . . . . 57 
Fig. 27. Radially integrated star production per proton versus longitudinal distance in concrete/soil . . . . . . . . . 58

Fig. 28. Cross section of tunnel showing primary and secondary shields . . . 62

Fig. 29. Schematic of SSC site and required lands . . . . . . . . . . 64

Fig. 30. Enlarged plan of the near cluster showing shielding vectors . . . . 65

Fig. 31. Comparison of CASIM and MARS at $10 \mathrm{TeV}$. . . . . . . . . . 78

Fig. 32. Comparison of CASIM and MARS at $20 \mathrm{TeV}$. . . . . . . . . . 79

Fig. 33. Comparison of FLUKA 82 with experiment at $200 \mathrm{GeV}$. . . . . . 80

Fig. 34. Comparison of CASIM with experiment at 400 and $800 \mathrm{GeV}$. . . . 81

Fig. 35. Comparison of CASIM and TOMCAT for muons at $240 \mathrm{GeV}$. . . . 82

Fig. 36. Comparison of TOMCAT muon spectrum with experiment at $240 \mathrm{GeV} 83$

Fig. 37. Muon shielding contours from MARS at $1 \mathrm{TeV}$. . . . . . . . . . 84

Fig. 38. CASIM muon momentum spectrum and experiment at $800 \mathrm{GeV}$. . 85

Fig. 39. Hydrological model of a domestic well near the tunnel . . . . . . 88 


\section{INTRODUCTION}

The Superconducting Super Collider laboratory or SSC is a unique complex of facilities for research in high energy physics, concerned with study of the most basic structure of matter and energy. The most important parts of the facility are an interconnected group of accelerators whose purpose is to generate beams of 20 $\mathrm{TeV}$ protons that are caused to collide at selected points. Detectors located at these collision points record the products of the collisions; detailed examination of these data reveal the fundamental building blocks of matter and the ways in which they interact.

The schematic plan view of the SSC shown in Fig. 1 indicates the size of the facility. The injector complex, a cascade of accelerators, feeds the main collider ring, 53 miles in circumference. The collider ring consists of a tunnel, 10 feet in diameter, in which are located two rings of superconducting magnets, as shown in Fig. 2. The protons leaving the injector at an energy of $1 \mathrm{TeV}$ enter one or the other beam tube within a ring of magnets and circulate around the 53-mile path. An accelerating system, labelled RF in Fig. 1, gradually accelerates the protons in each ring up to a final energy of $20 \mathrm{TeV}$. The beams of counter-rotating protons are held at $20 \mathrm{TeV}$ for many hours. They are brought into collision in several (4 initially) interaction regions (IRs). The numbers of protons are large enough that, even with a rather high collision rate at each IR $\left(10^{8}\right.$ interactions $\mathrm{s}^{-1}$ at the design luminosity of $10^{33} \mathrm{~cm}^{-2} \mathrm{~s}^{-1}$ ), the rate of loss of beam current is very small. Typically, the cycle of injection, acceleration, and storage for physics experimentation needs to be repeated once or twice a day.

The SSC facility is described in detail in the Conceptual Design Report [1]. A brief description, adequate for the purpose of the present report, appears in the DOE Invitation for Site Proposals [2].

The purpose of the present report is to:

1. Identify potential and actual sources of radiation during operation of the facility;

2. Provide technical information on the nature and magnitude of the radiation from those sources;

3. Describe the calculational tools available to track the deposition of the energy of the radiation and to translate the energy loss into radiation dose contours;

4. Use those dose contours to determine the magnitudes and directions (vectors) defining the containment regions, outside of which radiation levels are smaller than those found in everyday life. 

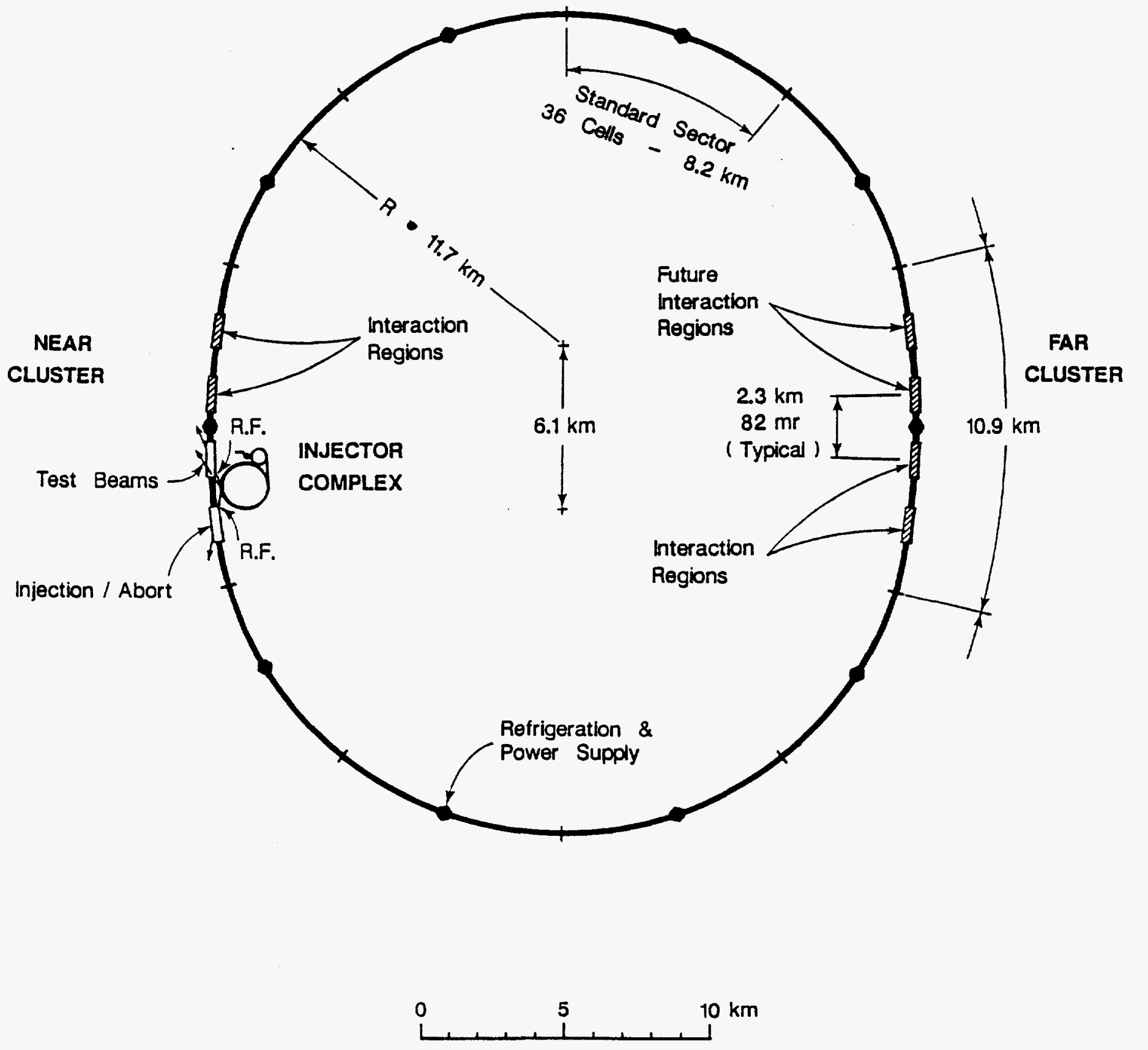

Figure 1. SSC collider ring layout. The clusters are joined by arcs of $11.7 \mathrm{~km}$ radius. The far cluster consists of four interaction regions separated by $2.3 \mathrm{~km}$. The near cluster has two interaction regions and two utility straight sections (open rectangles) for injection and abort and for acceleration (RF). The cascade of synchrotrons that form the injector is inside the main ring at the utility straight sections. There are 10 refrigeration and power units around the ring (black cllipses). 

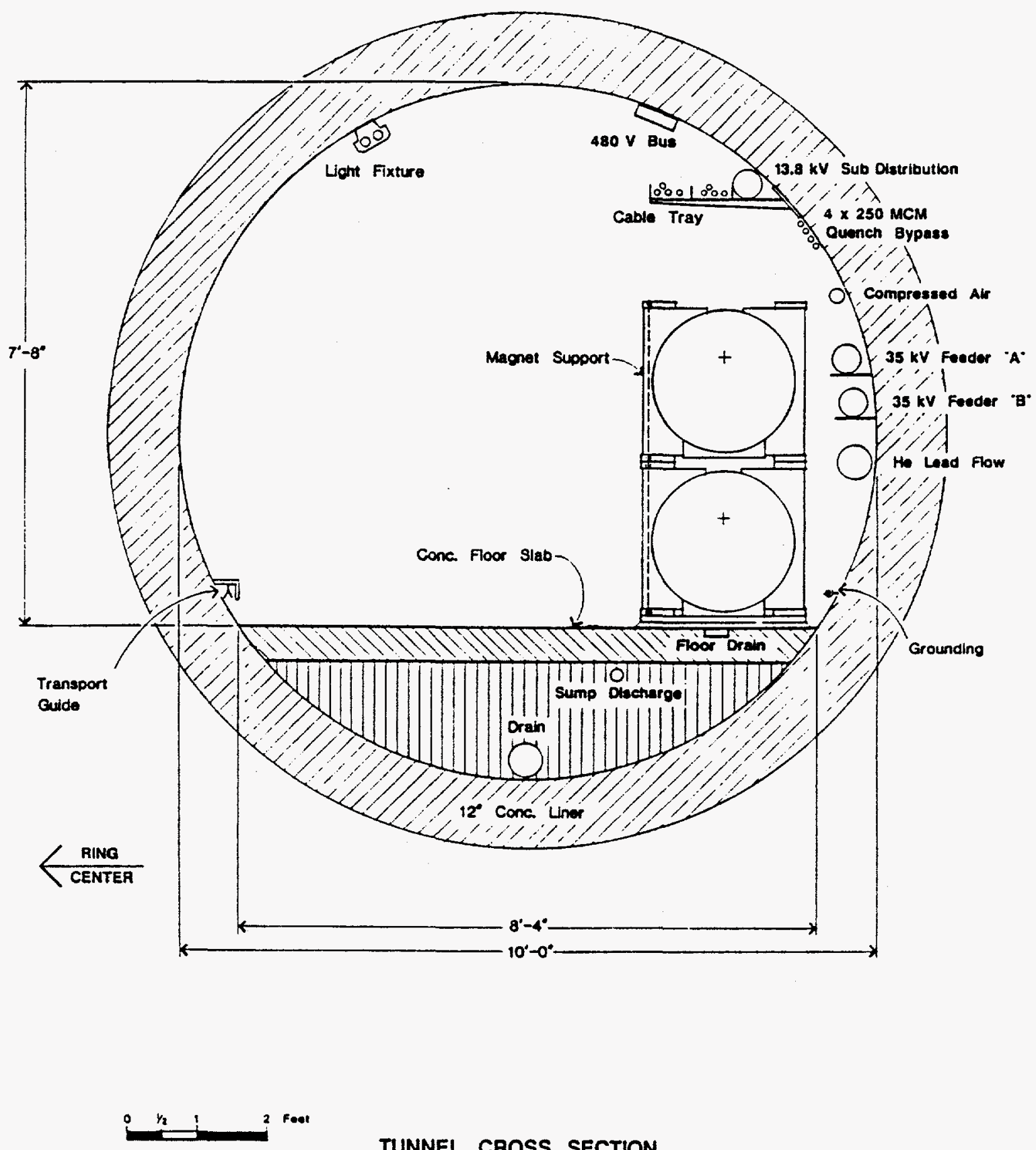

TUNNEL CROSS SECTION

Baem Separation $70 \mathrm{~cm}$

Figure 2. Collider ring tunnel profile showing the position of the two collider rings and the location of the utilities. 
The emphasis of the report is on the global shielding requirements of the SSC facility, that is, the large scale requirements related to land acquisition and shared use of lands adjacent to the complex. The detailed shielding requirements and other safety considerations (handling of cryogens, for example) in the tunnel and interaction regions are not discussed, nor are the requirements of test beams.

The Injector complex is a major accelerator facility in its own right. However, it is not discussed here since there is considerable operating experience with the comparable facilities at CERN and Fermilab. There are also studies for the HERA project under the city of Hamburg. These provide evidence that the SSC Injector complex poses no new radiological problems and that its safe operation can be assured by prudent design based on documented experience [3].

The SSC work on environmental radiation shielding had its origins in a workshop held by the Central Design Group in October, 1985 [4]. The documentation supplied at the workshop has formed the basis of much of the subsequent work.

\subsection{Sources of Radiation}

As a radiation source the $\mathrm{SSC}$ is similar to the projection tube of a television set, consisting of a beam of particles that can be steered by electric or magnetic fields and turned on and off. While gigantic in size compared with present facilities, the SSC is very similar in character to existing accelerator complexes at Fermilab near Chicago and the European Laboratory for Particle Physics (CERN) near Geneva, Switzerland. These laboratories and other facilities at Brookhaven National Laboratory, Stanford University, and Cornell University in the U.S. and institutions abroad routinely operate high-energy particle accelerators in a very safe manner. The benign nature of such facilities, from the points of view of both radiation and industrial hazards, is illustrated by the fact that Fermilab's site is completely open to access by the general public. Naturally, working portions of the Fermilab accelerator are restricted to employees and other qualified personnel, but the site above ground is safe for visitors at all times.

\section{(a) Interaction regions}

The SSC radiation consists of subnuclear particles initially moving with kinetic energies very much greater than the normal energy within atoms. During operation as a collider, the beams are brought into collision at several interaction points around the ring, as already stated. One or more detectors record the swiftly moving particles produced in the collisions and in the process absorb some of them. But some of the most penetrating particles and secondaries travel beyond the interaction regions. Thus the IRs are a source of radiation whose shielding must be assessed. 
(b) Beam dumps

As already mentioned, the main accelerator has a regular cycle of operation: injection, acceleration to $20 \mathrm{TeV}$, storage of colliding beams. At the end of a cycle, when collisions over many hours have degraded the beam quality sufficiently, the cycle is terminated by aborting the beams, that is, dumping each beam via an extraction system into a specially designed facility called a beam dump. The beam dump consists of heavy shielding and stopping material sufficient to contain completely the heat and induced radioactivity of the full $20 \mathrm{TeV}$ beam. (The abort system is described in Section 5.10, and the beam dump in Section 6.6, of Ref. 1.) The material of the beam dump serves as a target for the proton beams and so is a source of radiation consisting of secondary particles produced by the stopping of the primary protons. The regions labelled "abort" on Fig. 1 are the locations of this second source of radiation.

In normal operation, the aborting of the beam occurs infrequently, perhaps once or twice a day. During accelerator studies and machine improvement periods, however, the beams may be dumped more frequently. Generally, such aborts are insignificant from a radiation point of view because they are almost always of beams of less than full energy and intensity. Other aborts of the beam may occur when the beam sensors detect a malfunction and react to inhibit operation of the facility in a potentially unsafe condition. The frequencies of all the various types of beam aborts have been folded into the estimates of the source of radiation associated with the beam dumps.

(c) Beam cleanup regions

In both its acceleration and storage modes, the SSC proton beam is localized to a very small region near the center of the beam tube as it circles around and around. The overwhelming bulk of the beam is confined to a pencil region a few millimeters in diameter, but there is a small halo of particles making larger excursions from the beam axis. The quality of the beam and experimental conditions at the interaction regions are improved if this halo is scraped away. Specially designed beam cleanup stations contain scrapers to intercept the halo. These stops thus become targets for the protons in the halo and are sources of secondary particles.

(d) Accidental loss of full beam

While the interaction regions, beam dumps and beam cleanup regions are the only regular sources of significant radiation from the SSC, consideration must be given to accidental loss of the full beam anywhere around the 53-mile circumference of the machine. Such a major event will seldom, if ever, occur. Furthermore, such a loss would damage the accelerator itself and require lengthy repair. In 
discussing the dose contours for accidental loss of full beam, we therefore present the results per occurrence, whereas for the other sources we give average doses per year.

\subsection{Types of Radiation}

The protons in the beams of the SSC and the particles that they produce by collisions may have very high energies indeed, comparable to the high energy components of the Earth's cosmic ray background, a natural source of radiation that is incident continually on the Earth from deep space.

\section{(a) Hadrons}

Most of the initially produced particles are massive and strongly interacting (they are called hadrons). Some of them decay very rapidly in flight into lighter particles. Others collide with matter and are transformed into tertiary hadrons of lower energy or are brought to rest, where they decay quickly into the familiar, stable particles (electrons, protons, neutrons) that make up the common materials all around us. The distances of travel are scaled by the interaction length (the mean distance traveled in a material before a nuclear collision). For light materials, such as graphite, aluminum, or concrete, the interaction lengths of different hadrons are all about 40 to $60 \mathrm{~cm}$. For heavier materials like iron, the interaction length is roughly $20 \mathrm{~cm}$. Hadronic radiation is degraded rapidly in each successive interaction length. It is stopped completely in a few meters or tens of meters, depending on the primary energy, the type of radiation, and the density of the absorbing material. The physical processes involved in the degradation of the radiation are well understood both theoretically and experimentally and these mechanisms are built into the calculational tools described in Chapter 2.

(b) Photons and electrons

Accompanying the hadrons are high energy photons and electrons. These particles degrade only via electromagnetic interactions, but are absorbed very rapidly in matter, nevertheless. The characteristic distance at high energies is called a radiation length. It depends strongly on atomic number. For graphite, it is about $20 \mathrm{~cm}$; for iron, less than $2 \mathrm{~cm}$. As a consequence, the degradation of electrons and photons (called the electromagnetic cascade) occurs over distances of the order of a few meters in solid materials.

(c) Muons

Special consideration must be given to one type of particle, called muons. Muons are heavy electrons, identical in all respects except mass. Muons are a 
rare component ( $a$ few tenths of a per cent) of the radiation created in the SSC, but, in contrast to the hadrons, photons and electrons, they are not readily absorbed at high energies. The elementary explanation, somewhat over-simplified, is that radiative energy loss processes are proportional to the inverse square of the mass of the incident particle. Even though radiative processes are not the only mechanisms of energy loss, the muon, with a mass 200 times larger than an electron, loses energy far less rapidly than an electron or hadron and so penetrates to greater distances. The most energetic muons travel several kilometers in earth. The muons eventually stop and decay after about two microseconds into electrons (and essentially non-interacting neutrinos). Experience at existing accelerators and from the cosmic rays show that the high energy muons are very strongly collimated along the direction of the proton beams initiating the collisions that produce them. The shielding considerations thus apply to long regions tangential to the circumference of the main ring in either direction at the interaction regions and down stream of the abort regions shown in Fig. 1.

\section{(d) Residual radioactivity}

Radioactive nuclei are produced in the process of stopping high energy particle beams. In the course of stopping, the energetic particles produce numbers of lower energy (shower) particles in the absorber, and these in turn slow down and stop. Low energy (slow) neutrons are produced in the shower and these particles are absorbed by the nuclei of the surrounding matter. Some of the created nuclei are unstable with radioactive half-lives ranging from microseconds to thousands of years. In general, the radioactive products in the lighter absorbers (carbon, oxygen, helium) decay rapidly and exhibit little long-lived radioactivity. A few of the nuclei produced in heavier materials (copper, iron, lead, etc.) can have lifetimes as long as a year or more. These nuclei are similar to those responsible for the natural radioactivity that we have all about and within us.

The artificially induced radioactivity produced in the SSC accelerator complex is mostly of very low level. The few places where higher activation is produced are mainly the beam abort dumps. As already described, these dumps are isolated, specially designed facilities whose purpose is to contain all induced radioactivity and safely dissipate the heat generated.

(e) Synchrotron radiation

One other type of radiation deserves mention-the so-called synchrotron radiation. Hitherto only relevant for circular electron accelerators, synchrotron radiation is a factor in the design of the SSC. The energy is sufficiently high that the protons emit enough synchrotron radiation to put demands on the cryogenic cooling system for the superconducting magnets. The conceptual design param- 
eters give an estimate of approximately $18 \mathrm{~kW}$ of synchrotron radiation emitted uniformly around the machine. The radiation consists of visible and ultraviolet light and soft $x$ rays with a critical energy of $280 \mathrm{eV}$. While the refrigeration and vacuum systems must be designed to handle its consequences, the synchrotron radiation is completely absorbed in the wall of the beam tube inside the magnets. It is of no concern for radiation shielding.

\subsection{Further Discussion of Sources and Shielding}

\section{(a) Different sources}

In normal operation, the proton beams are confined within the vacuum chambers of the magnet rings. Radiation occurs only at the interaction regions where the two beams cross one another, at the beam cleanup regions, and at the primary beam-abort dumps where the beams are absorbed at the end of a machine use cycle. A very small amount of radiation, which is absorbed within the walls of the tunnel, is produced uniformly around the ring by the collisions of beam protons with residual gas molecules in the evacuated beam tubes. The beam current will decrease by about $20 \%$ over a period of 12 to 24 hours because of beam-beam and beam-gas interactions; the remaining $80 \%$ of the beam will be ejected in a 300 microsecond burst to the beam-abort dumps at the end of the cycle. The $\leq 8 \%$ loss around the ring by residual gas scattering is a local shielding and operations safety issue, but is not significant for this report because the loss is small and not localized.

Radiation in the transverse direction from the beam interaction points is dominated by hadrons; in the forward direction it is dominated by muons. This is because the forward hadrons are absorbed within the accelerator lattice by special scrapers and absorbers designed to protect the superconducting magnets. Beyond the absorber region and outside the beams enclosure, the radiation is a highly collimated muon cone that is produced in the decay of short-lived particles from the interaction.

The radiation from the primary beam-abort dumps is qualitatively the same as that around the interaction regions, but the shielding presents a different problem. At an interaction region, the shielding must be designed around the collision point and be removable. The primary beam dumps, on the other hand, have no requirement for ready access. Because they absorb the energy of most of the residual protons in a very short time, the beam dumps must, however, be designed to permit dissipation of the associated thermal and mechanical shocks, in addition to reducing the radiation to permissible levels. The dumps require appreciable land downstream from the abort regions, indicated by the arrows in Fig. 30. The requirements are described in Chapter 4. 
The radiation from the beam cleanup regions is similar in character to that from the primary beam dumps, but involves only a small fraction of the total beam.

(b) Inputs for shielding calculations

The primary parameters of the SSC, as described in the Conceptual Design Report [1], are two beams of $20 \mathrm{TeV}$ protons, each of a current $I_{0}=73 \mathrm{~mA}$ $\left(N_{p}=1.3 \times 10^{14}\right.$ protons in each ring), colliding with a maximum luminosity of $10^{33} \mathrm{~cm}^{-2} \mathrm{~s}^{-1}$ (corresponding to a maximum interaction rate of $10^{8} \mathrm{~Hz}$ at each IR). For the purpose of prudent determination of the shielding requirements, it was decided to assume that the luminosity could be ten times as high as design. The parameters used in this report are therefore

$$
\begin{aligned}
& N_{p}=4 \times 10^{14} \text { circulating protons/ring } \\
& \mathcal{L}_{\max }=10^{34} \mathrm{~cm}^{-2} \mathrm{~s}^{-1} \\
& \text { Collision rate }=10^{9} \mathrm{~Hz} / \mathrm{IR} .
\end{aligned}
$$

These are the determining parameters for calculations concerning the IRs and also the accidental loss of beam.

The totality of beam scraped off in the various beam cleanup regions could amount to several percent, but this will not be done at just one location. Downstream of a scraper there are scraped protons impinging for tens of meters on the magnets and other accelerator components. These protons and their secondaries are sources of heat. For scrapers located in the arcs, the presence of superconducting magnets sets a limit on the heat input and therefore on the amount of halo removal that can be done at such locations. A design upper limit is $0.2 \%$ of the beam over a storage cycle at $20 \mathrm{TeV}$. More extensive beam cleanup stations must be located in the utility straight sections adjacent to the injector complex, where the rf, injection and abort units are located. There, conventional magnets, which can handle a larger heat load, can be used downstream of the scrapers. The dose equivalent calculations are presented for $0.2 \%$ (for beam cleanup on the arcs) and $1.0 \%$ beam loss from scraping. Scaling to other values can be done on the figures.

For the intermittent aborts of the primary beam an estimate of the annual number of such events is necessary. As already mentioned, there are a variety of reasons for aborting the beam. The routine cycling of the machine is estimated to occur once every 12 to 24 hours. With 6000 hours $\mathrm{yr}^{-1}$ of operation of the facility for physics research, this corresponds to about 300 beam aborts per year at full energy and intensity. Accelerator studies will involve many more than this number, but generally of lower intensities or intermediate energies. Consideration 
of such beam aborts from the point of view of production of radiation leads to the conclusion that they are equivalent to not more than an additional 200 dumpings of the full beam at $20 \mathrm{TeV}$. In this report we take 500 full-energy full-current beam aborts per year as the equivalent source from the beam dumps.

(c) Radiation dose criteria and their consequences

The transverse and longitudinal dimensions that are the end product of these studies are based on a safe maximum permissible annual dose equivalent at the perimeter of the site and beyond. The chosen value is based on relevant governmental regulations and further allowances during design and operation to provide additional assurance in the matter for the general public.

In defining radiation criteria for the protection of the public and the environment, the Department of Energy recognizes the recommendations of the National Council on Radiation Protection and Measurements (NCRP) and the International Commission on Radiation Protection (ICRP). The relevant DOE Order (in draft form), which is currently labeled 5480 .XX, defines the radiation standards for the protection of the public in the vicinity of DOE facilities. The permissible level for continuous exposure is limited to $100 \mathrm{mrem}$ per year of whole-body radiation. This is to be compared to an average background level in the continental United States of 100 to 200 mrem per year due to naturally occurring radiation. For non-continuous exposure the limit is 500 mrem per year. In practice, DOE specifies that facilities must be designed to achieve radiation dose equivalents as low as reasonably achievable (ALARA) below the defined limits. The shielding for the SSC will be designed for consistency with this ALARA policy. The SSC facility shall have the capability, consistent with the types of operations conducted, to monitor routine and non-routine releases to the environment, and to assess doses to members of the public and impacts on the environment to ensure compliance with these standards.

The average annual dose equivalent chosen here to define the land requirements is $10 \mathrm{mrem}$. This ALARA design value is $10 \%$ of the DOE upper limit, chosen in recognition that there is some uncertainty in the extrapolation of the shielding calculations to SSC energies.

The calculations described in this report show that adequate radiation shielding (to less than 10 mrem per year) in the transverse direction at the interaction regions and the primary beam dumps is afforded by 9 meters ( 30 feet) of light earth or equivalent beyond the tunnel (soil density $1.8 \mathrm{~g} \mathrm{~cm}^{-3}$ ).

The muon shielding needs have been determined by a conservative approach. While the basic absorption mechanisms are well understood, the exact level of production of energetic prompt muons at SSC energies is somewhat uncertain. 
This is not surprising. Indeed, some of the important research to be done with the SSC is to investigate new phenomena that will undoubtedly have energetic muons as by-products. The new phenomena themselves are sufficiently rare that they are not a significant source of radiation. But the accompanying more frequent component (sometimes called background by the physicists) will have muons present. Fortunately, existing data at present energies and relatively reliable theoretical models permit extrapolation of the muon yields to SSC energies, accurate to within a factor of three. With a conservative upper bound on the production, the calculations show more than adequate shielding is given by muon shields that extend tangentially for 2.3 to $5.2 \mathrm{~km}$ downstream from the various muon source points. Because of the gentle curvature of the ring, such distances correspond to far smaller distances transverse to the ring - see Table IV-1.

As discussed in (b) above, while the majority of beam cleanup will occur in the utility straight sections, some scraping will occur in the arcs and be sources of radiation. To provide adequate shielding for such sources, the hadron shielding has been conservatively specified to be at least 9 meters $(30 \mathrm{ft}$.) everywhere around the tunnel, not just at the IRs and beam dumps. The corresponding forward muon cone extends tangentially along the ring for a distance of 2.3 kilometers. The sweeping of this cone around the entire periphery delineates a possible muon radiation zone of 225 meters ( 740 feet) extent measured radially outward from the tunnel. The muon zone lies in the plane of the collider rings. Since muons are far less interactive per unit length than hadrons, the muon zone demands less restrictive personnel access conditions than the $10.5 \mathrm{~m}$ radius hadron zone.

\subsection{Preview of the report}

The body of this report is devoted to giving the technical basis for the key shielding parameters as they pertain to the needed land acquisition and minimum tunnel depth for the SSC. Chapter 2 describes the tools available for the calculations of absorption of radiation in matter and generation of dose contours. Intercomparison of different tools and comparisons of the calculations with observations at existing accelerators are given in Appendix B. The results of the calculations for hadrons and muons from the different sources in the form of dose plots appear in Chapter 3, as well as a discussion of the accuracy of the calculations. Also appearing in Chapter 3 are a discussion of the unlikely accidental loss of the full beam and a treatment of the potential activation of ground water in wells in the muon radiation zone and of possible activity in water supplies from beam dump operation. Chapter 4 is an analysis of the distances derived from the dose contour graphs leading to specification of the key shielding parameters in terms of vectors and widths from the source points around the ring. Translation 
of these key dimensions and directions into land area of different sorts is done in the DOE Invitation for Site Proposals [2]. The report closes with a short section of conclusions. 


\section{COMPUTATIONAL TOOLS}

Radiation hazards at high energy accelerators are largely due to ionization produced by the interactions of photons, electrons, hadrons, muons, and, indirectly, to neutrons. Except under some circumstances involving muons, most of the ionization is caused by the numerous low-energy products of cascade processes. High-energy particles (e.g. $20 \mathrm{TeV}$ protons, in the case of the SSC) collide with other beam particles or with stationary matter. The products of these primary collisions then collide with matter, etc., until all of the primary energy has been dissipated by ionization. Tens to hundreds of thousands of particles in each cascade are sufficiently energetic to merit attention. We are interested in the energy deposition some distance away. Obviously, the result is dependent upon the intervening arrangement of walls, tunnels, iron magnet yokes, and the materials used. The difficulty of the problem is compounded by the fact that we are interested in the result at a place where the dose is many factors of ten less than that near the primary collision point. Hence results of model calculations are especially sensitive to small errors in the model, although the exponential falloff in the propagating energy means that shielding distances depend only logarithmically on the results.

Several Monte Carlo programs have been developed to make detailed simulations of these processes. Each has evolved over a decade or more, and all have grown and been modified as energies have increased and more data and better models have become available. In addition, innovations have been freely adopted. For example, CASIM, the Fermilab code developed by Van Ginneken, uses weighting techniques to reduce dramatically the computing time with little loss in overall accuracy [5]. As energies and complexity have increased, other codes have incorporated similar features. Even though they are not completely independent, the way the codes model collisions, follow the collision products, and handle the geometry are sufficiently different that comparisons are useful. Their general agreement justifies having confidence in the results. (See Appendix B.)

Even though much of our information comes from detailed modeling using these codes, there is still a need to understand scaling with distance, shielding thickness, and incident beam energy in a more analytic way. The model developed by Moyer [6] in the course of Bevatron upgrades 25 years ago still serves this function. This phenomenological approach is based on the observation that the propagating particles are mostly fairly energetic neutrons. Neutrons with kinetic energies above about $150 \mathrm{MeV}$ have a nearly constant interaction length in shielding, and below this energy the interaction length decreases rapidly. Even though the relevant dose is due to low-energy neutrons, these are the local progeny of higher energy neutrons whose attenuation is easily describable. Lindenbaum 
later suggested[7] that scaling with beam energy could be approximated as a power law $\left(E^{m}\right)$, where $m$ is somewhat less than one. This approximation has survived the enormous increase of beam energies since it was first proposed, and the present best value for the exponent is $m=0.80 \pm 0.10$ [8].

\subsection{Available Cascade Simulation Codes}

The primary work for this report has been done using CASIM, the Fermilab code mentioned above. Comparisons and additional calculations have been made using MARS10 and FLUKA82, and, to a lesser degree in the present case, HETC. We briefly describe each of these codes:

1. CASIM [9]. Hadronic production in hadron-nucleus collisions is via a modification of the Hagedorn-Ranft thermodynamic model[10]. Hadrons are followed if their momentum exceeds $300 \mathrm{MeV} / \mathrm{c}$. In each step only one of the collision products is followed, with appropriate weighting. Electromagnetic showers are either included in an average way or with explicit coupling to the shower code AEGIS [11]. Recent versions include charged particle energy loss through hard bremsstrahlung, pair production, and photonuclear interactions. These processes become dominant at energies above $1 \mathrm{TeV}$ or so, and contribute to angular spread as well. Geometry is specified through a user-supplied FORTRAN program. Magnetic fields are easily included.

For primary collisions at $20 \mathrm{TeV} \times 20 \mathrm{TeV}$, hadron multiplicities may be underestimated by about a factor of three. For most applications this is of little consequence, since the eventual dose has more to do with the total energy in the cascade than with the details about how rapidly the multiplicity increases near the beginning. An exception concerns the muon dose. Fewer hadrons of higher energy lead to more hard muons from direct production, meson decay, and muon pair production early in the shower development.

A modification of CASIM includes muon production and transport [12].

2. MARS [13]. In this Serpukhov-developed code, hadron inclusive spectra (using a selection scheme similar to that of CASIM) are described using an additive quark model of hadron-nucleus interactions for Feynman $x>0$, and a phenomenological model for $x \leq 0$. Semi-empirical formulas are used to simulate low-energy particle production. Magnetic fields can be included. Electromagnetic showers are handled using a modified version of AEGIS. Hadrons with energies above $50 \mathrm{MeV}$ are transported, and incident energy up to $30 \mathrm{TeV}$ can be accommodated.

* The CERN code GEANT also correctly transports muons, but it is rather specialized for detector simulations. 
3. FLUKA [14]. In contrast to CASIM and MARS, the CERN program FLUKA makes a full analog simulation of the cascade. Unless biasing options are specified, all particles down to $50 \mathrm{MeV}$ kinetic energy are followed, including electromagnetic showers if the EGS [16] inclusion option is used. Below $5 \mathrm{GeV} / \mathrm{c}$, the particle production model describes inelastic collisions as quasi-two-body processes producing resonances which subsequently decay, while above this energy a multichain dual parton fragmentation model is employed. A large body of parameterized data is used to describe the cross sections and hadron-nucleus collisions. A substantial upgrade to FLUKA86 has been made[15]; with some minor changes it is now available as FLUKA87.

While description of the primary collision defaults to the multichain fragmentation model, FLUKA can be interfaced to a user-supplied production model such as PYTHIA [17] or ISAJET [18]. (These state-of-the-art primary collision codes are described below.)

Its geometry package has been supplemented by the combinatorial geometry package from MORSE[19], the low-energy neutron-photon transport code developed at Oak Ridge National Laboratory (ORNL).

FLUKA occupies more memory and takes longer to execute than either MARS or CASIM, but on the other hand does its simulation in a detailed and independent way, based upon current physical models.

While CASIM results form the bulk of this report, crosschecks between CASIM and the CERN code FLUKA82 have been made, some of which are described in Appendix B.

4. HETC [20]. This workhorse High Energy Transport Code was developed at ORNL to follow hadronic cascades through the resonance region, and has found wide application in detector simulations. Hadron-nucleus collisions below $3 \mathrm{GeV}$ are described using an internuclear cascade model parameterized to fit the available data; above $3 \mathrm{GeV}$ the current version refers to the appropriate FLUKA subroutines. The descriptions of particles falling below $50 \mathrm{MeV}$ is written onto tape, for input to MORSE, which can follow neutrons all the way into the thermal region if so desired. HETC/MORSE is the best available way to simulate the low-energy $(0.1-10 \mathrm{MeV})$ neutron flux in an accelerator environment. 


\subsection{Particle Production Codes}

The center of mass energy for a $20 \mathrm{TeV}$ proton colliding with a nucleon at rest is $194 \mathrm{GeV}$. Collisions at this energy, which is less than half that available at the $\mathrm{S} \overline{\mathrm{p}} \mathrm{S}$, are adequately described by the several models available in the cascade simulation programs described above. However, the primary interactions of protons in the colliding beams are not. Moreover, the modeling of these collisions is being rapidly improved as new theoretical ideas, data, and computing algorithms become available. The present state of the art consists of the Monte Carlo programs PYTHIA [17] and ISAJET [18]. FLUKA has already been modified to use PYTHIA-generated events for the first collision, and it is hoped that HETC and CASIM will soon incorporate similar features. We briefly describe the two programs, and then make some comparisons between them.

\section{(a) ISAJET}

ISAJET, developed by Frank Paige and his co-workers at Brookhaven National Laboratory, is a Monte Carlo program that simulates $\mathrm{p}-\mathrm{p}$ and $\mathrm{p}-\overline{\mathrm{p}}$ collisions at high energies. It is based upon perturbative QCD cross sections, plus leading order QCD radiative corrections for initial and final state partons, and phenomenological models for jet and beam jet fragmentation. In its present form, it does not include diffractive and elastic scattering processes. In generating an event, it proceeds in four distinct steps, incorporating both perturbative and non-perturbative QCD and nonperturbative models for hadronization:

1. A primary hard scattering is generated according to available cross sections combined with nonscaling structure functions.

2. QCD radiation is added from both initial and final partons.

3. Hadrons are produced from each parton using the independent fragmentation model.

4. Hadrons from the spectator beam jets are added, assuming that these are indistinguishable from events at a lower energy with no hard scattering at all.

\section{(b) PYTHIA}

PYTHIA is the present version of the Lund Monte Carlo program for hadronic processes. At its core are a long list of perturbative QCD matrix elements for two-body processes: $q_{i}+q_{j} \rightarrow q_{i}+q_{j}, \bar{q}_{i}+g \rightarrow \bar{q}_{i}+g$, etc. As with ISAJET, much of the list consists of exotica such as $W^{ \pm}$and Higgs production. While these are primary reasons for building a Supercollider, it is the more prosaic processes that create radioactive background. Initial and final state radiation 
are included. Hadronization occurs by color-string fragmentation. Sjöstrand's model [21] describing the several parton interactions that occur when the hadrons pass through each other has been incorporated to avoid divergence in the hardscattering matrix elements for small $p_{\perp}$, and the continuity between low- $p_{\perp}$ and high- $p_{\perp}$ scattering is quite good. Elastic and diffractive scattering have been included, using the parameterizations of Block and Cahn [22] and Goulianos [23].

(c) Differences and Reliability

Both codes have gradually included such features as gluon radiation by incoming and outgoing partons. A major remaining difference is in the non-perturbative fragmentation models, independent fragmentation for ISAJET and color-string fragmentation for PYTHIA. Both models seem to lead to beam jet multiplicities below those measured at the $S \overline{p p S}$, although the authors claim each model can be tuned to yield agreement.

Although there are differences between ISAJET and PYTHIA, these two codes currently provide the most accurate simulation of very high energy $p-p$ collisions. Neither program is clearly superior to the other at present.

\subsection{Muons}

As discussed in Chapter 1, muons merit special attention. Muons with energies of 1 or $2 \mathrm{TeV}$ may travel through a kilometer or more of soil before stopping. Above $1 \mathrm{TeV}$, the dominant stopping mechanisms are bremsstrahlung and pair production, processes that are well understood [24], but that often involve large, discontinuous changes in the muon energy, rather than the almost continuous, gradual loss of energy by atomic collisions. As a consequence, the transport of muons requires a somewhat different approach from the diffusion approach to hadronic cascades. Only a modified version of CASIM treats energy loss fluctuations in muon transport [12]. It also includes production from a variety of sources:

1. Direct production. So-called prompt muons, mainly the decay products of charmed mesons, are rather poorly modeled at present. (This problem is discussed in more detail below.) Since heavy quarks have a comparatively hard production spectrum, direct production is a dominant source of energetic muons.

2. Decay of primary pions and kaons. If sufficient time elapses between production and interaction, a significant flux of muons can be produced from the decay of pions and kaons. In matter (as in the beam loss case) the mesons undergo hadronic collisions after such a short distance that decay is improbable, and decay muons do not contribute appreciably to the total 
flux. In the interaction regions, energetic mesons produced at small angles may travel of the order of $100 \mathrm{~m}$ before being swept out of the beam pipe.

3. Muon pairs produced by prompt gamma rays (from $\pi^{0}$ decay). Although these pairs are produced in matter struck by the gamma rays, the rate is independent of shielding configuration.

4. Secondary processes in the hadronic cascade. All of the process mentioned above recur in subsequent cascade generations. Since energies are lower, the contributions are to softer parts of the muon spectrum.

An example illustrating the relative importance of these contributions is shown in Fig. 3, as calculated by Van Ginneken[25]. Debris from the interaction region was allowed to travel through vacuum for $80 \mathrm{~m}$, after which it entered soil with a density of $2.24 \mathrm{~g} \mathrm{~cm}^{-3}$. These results can easily be scaled to different distances to the soil wall, since the contribution of pion, kaon, and muon decay scales linearly with the free space distance while other contributions are independent of the distance.

The muon flux deep in a shield depends on the primary pion and kaon and prompt muon production spectra. As mentioned above, the charged hadron multiplicity in CASIM is low, probably by a factor of three. While this should have little impact on hadronic dose calculations, it does mean that an unrealistically hard decay muon spectrum is produced. This contribution to the muon flux at large distances is therefore conservatively overestimated in the calculations cited in this report.

In estimating prompt muon production, Van Ginneken, Yurista, and Yamaguchi [26] multiply their assumed pion production spectrum by a $\mu / \pi$ ratio given by Ritchie et al. [27]:

$$
\mu / \pi=\left\{\begin{array}{ll}
10^{-4}(-1.92+\ln \sqrt{s})\left(1-2 x_{F}\right) & \text { for } x_{F}<0.4 \\
10^{-4}(-1.92+\ln \sqrt{s})\left(1-x_{F}\right) / 3 & \text { for } 0.4 \leq x_{F} \leq 1.0
\end{array} .\right.
$$

Here $\sqrt{s}$ is the total center of mass energy. The resulting differential spectrum is shown by the solid curve in Fig. 4 for $20 \mathrm{TeV}$ colliding beams. For comparison, F. Paige has made ISAJET calculations of the same distribution [28]. He finds $\approx 2 \mathrm{mb}$ for the total $\mathrm{D} \overline{\mathrm{D}}$ production cross section; after the inclusion of other heavy quark production and the proper branching ratios, he finds an effective total cross section of $0.5 \mathrm{mb}$ for prompt muon production. This cross section and a total inelastic cross section of $75 \mathrm{mb}$ were used to normalize the distributions shown by the histogram in Fig. 4. Van Ginneken's model predicts muons in $1.4 \%$ of the inelastic events, while ISAJET yields $0.3 \%$. Moreover, the ISAJET 


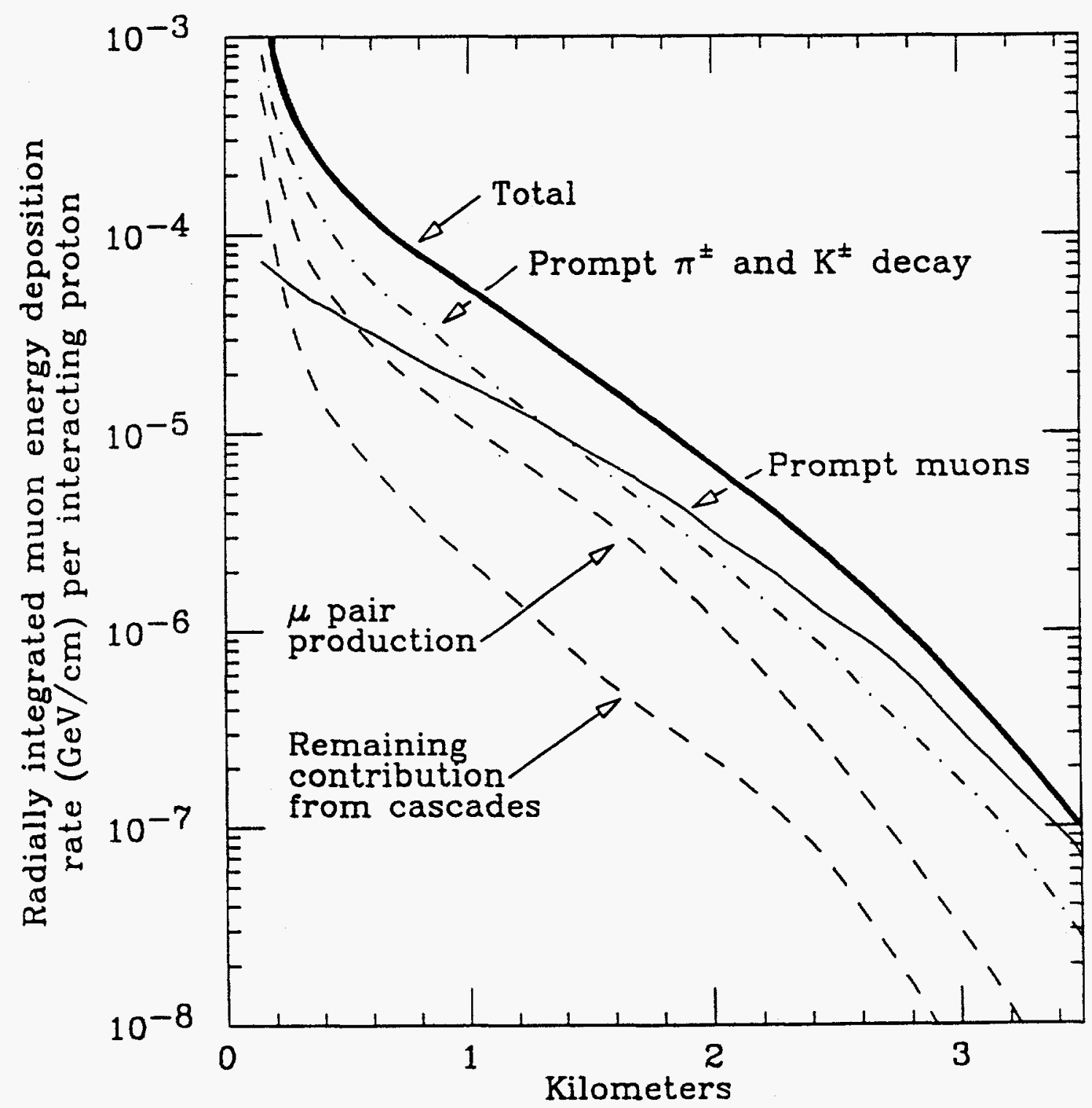

Figure 3. Radially integrated muon flux downstream of a $20 \mathrm{TeV}$ colliding beam interaction point, showing contributions of various muon sources as modeled by Van Ginneken. All primary interaction products are transported through $80 \mathrm{~m}$ of vacuum before they enter soil with a density of $2.24 \mathrm{~g} \mathrm{~cm}^{-3}$. The conversion to muon flux is made assuming $\langle d E / d x\rangle=2.26 \mathrm{MeV} \mathrm{g}^{-1} \mathrm{~cm}^{2}$. 


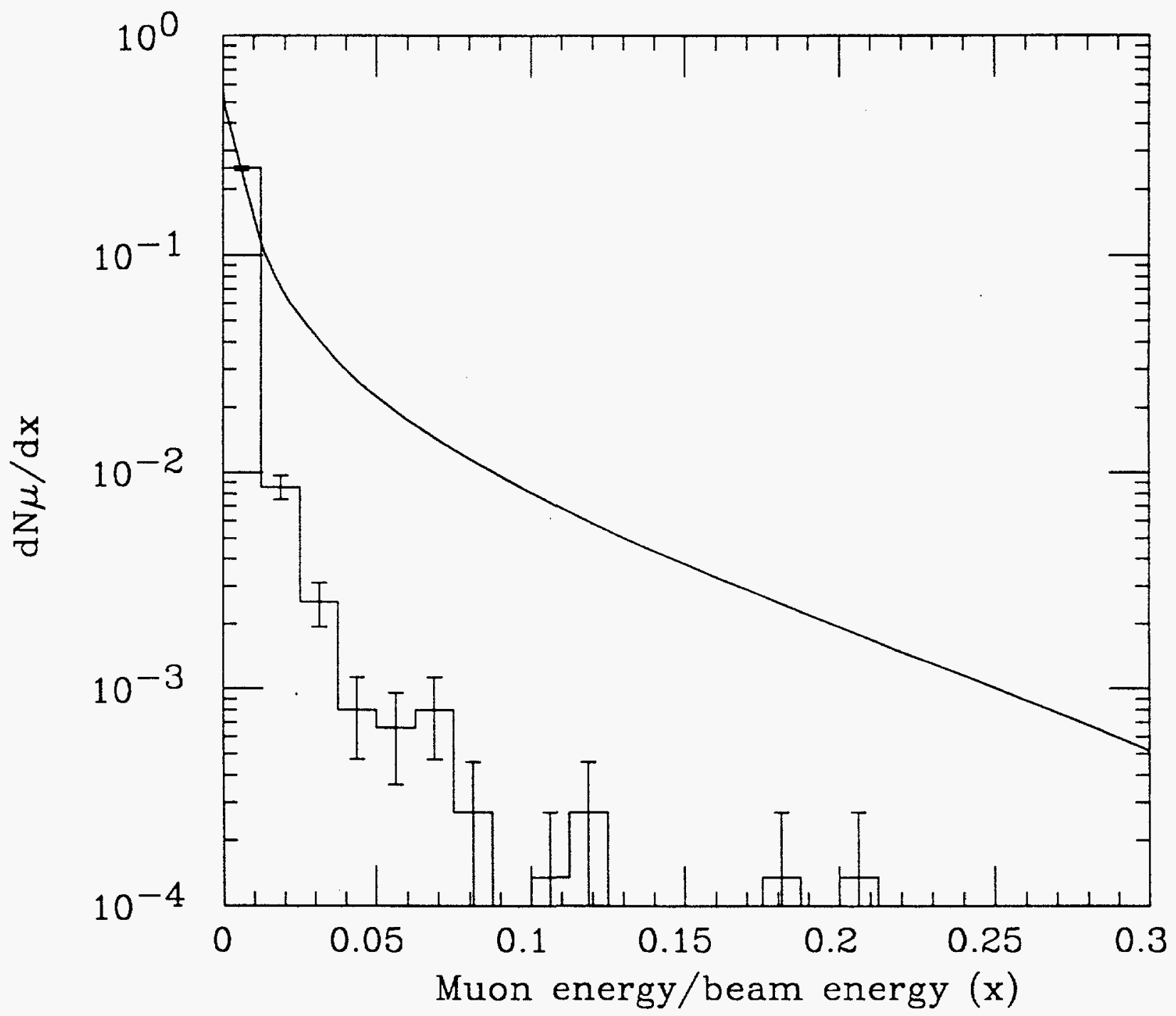

Figure 4. Prompt muon spectra for $20 \mathrm{TeV}$ colliding beams, as assumed by Van Ginneken (solid curve) and as calculated by Paige using ISAJET (histogram). An inelastic cross section of $75 \mathrm{mb}$ was assumed in normalizing the ISAJET results. 
distribution is substantially softer, partly as a consequence of a more realistic multiplicity. At $1 \mathrm{TeV}$ (corresponding to a range of about $1 \mathrm{~km}$ ), the ISAJET flux is lower by a factor of more than 20. Since the muon doses quoted in this report are based on Van Ginneken's model, we conclude that (a) total direct muon fluxes are overestimated by a factor of perhaps four, and (b) the high energy flux is overestimated by at least an order of magnitude. Although Paige urges caution in trusting the current ISAJET results, it seems evident that Van Ginneken's results represent conservative upper limits.

Muon transport at sub- $\mathrm{TeV}$ energies is treated in a very different way in Stevenson's TOMCAT [29]. Fluctuations do not dominate below $1 \mathrm{TeV}$ in soil, and by neglecting them he is able to solve the transport problem analytically. QED processes are all included, but in an average way. Scattering is considered to be the result of many small scatterings. Checks of this program and the transport parts of CASIM have been done at SpS energies [30].

\subsection{Definitions of Absorbed Dose and Dose Equivalent}

The absorbed dose of ionizing radiation is defined as the energy absorbed per unit mass. Until recently, the standard unit has been the rad, where

$$
1 \mathrm{rad}=100 \mathrm{ergs} \mathrm{g}^{-1}
$$

While still widely used, it has in principle been replaced by its SI equivalent, the gray, where

$$
1 \mathrm{~Gy}=1 \text { joule } \mathrm{kg}^{-1}=100 \mathrm{rad} .
$$

Not all radiation has the same ability to inflict biological damage. Stopping protons, for example, produce very dense localized ionization that results in more biological damage than does the same energy deposition by fast electrons. The dose equivalent takes this into account by multiplying the absorbed dose by an appropriate quality factor $(Q)$. Dose equivalent is often measured in rems:

Dose equivalent in rems $=Q \times$ absorbed dose in rads .

The quality factor is defined as unity for photons ( $x$ rays, gamma rays) and electrons ( $\beta$ rays). It can be as high as 20 for alpha particles from radioactive decay, and is about 3 for thermal neutrons. The SI replacement is the sievert, so that the dose equivalent in sieverts is $Q$ times the dose in grays.

Since low-energy radiation can be attenuated quickly as it penetrates tissue, one usually quotes the surface dose, or surface dose equivalent-i.e. the dose or dose equivalent near the surface, where it is maximal. 
Dose and dose equivalent are the quantities appropriate to this report, since we are dealing with radiation of several types. In the case of $x$ rays the appropriate quantity is exposure, which is a measure of the ionization produced in air by electromagnetic radiation. The unit of exposure is the röntgen (R), or in SI units simply coulomb $\mathrm{kg}^{-1}$.

\subsection{Conversion of Simulation Results to Radiological Units}

The available cascade simulation codes generate the density of hadronic interactions (number of "stars" per unit volume) at a given position in a material. Given knowledge of the composition and spectra of particles at this position, one can convert the star density to absorbed dose and dose equivalent. The procedure is somewhat simplified by the observation that there exists an equilibrium momentum spectrum of the participating hadrons at sufficiently large depths and distances from the shower core-conditions that certainly obtain for the situations of interest in this report. In this case a unique factor can be found that relates star density to radiological units. The determination of such a factor was reported in the systematic cascade studies of Van Ginneken and Awschalom [5] using the CASIM program [9].

The programs allow stars to be produced if the incident hadron is above some cutoff energy or momentum. In the case of FLUKA the cutoff is usually at a kinetic energy of $50 \mathrm{MeV}$. CASIM is usually run with a momentum threshold of $300 \mathrm{MeV} / \mathrm{c}$, corresponding for neutrons and protons to roughly the same energy cutoff. The simulations yield information on hadron spectra above the cutoff energies. In estimating the conversion factors to dose equivalent, assumptions must be made concerning the composition and spectrum at lower energies. Stevenson has observed that the lateral spectrum consists almost entirely of neutrons [31]. Van Ginneken and Awschalom normalized the neutron spectrum determined by Gabriel and Santoro[32] to the CASIM spectrum between $300 \mathrm{MeV} / \mathrm{c}$ and 800 $\mathrm{MeV} / \mathrm{c}(50 \mathrm{MeV}$ and $300 \mathrm{MeV})$ to extend their results to lower energies. They used the MADE concept of dose equivalent [33]. For concrete this led to the conversion factor $9 \times 10^{-6}$ rem star-1 $\mathrm{cm}^{3}$ reported in Ref. 5 and used in Ref. 26, the source of many of the results in Chapter 3.

The matter has recently been reexamined by Stevenson [34]. He uses O'Brien's neutron spectra[35], again normalized to simulation data between $50 \mathrm{MeV}$ and $300 \mathrm{MeV}$, to extend the simulation results to lower energies. He also made use of the ambient dose equivalent recently recommended as the operational quantity in radiation protection by the International Commission on Radiation Units [36]. A series of new FLUKA calculations were used to find contributions of neutrons, protons, pions, and photons from $\pi^{0}$ decay above $50 \mathrm{MeV}$, for a variety of targets 
and incident proton beam energies. About $2 / 3$ of the neutron contribution to the dose equivalent was found to come from neutrons with energies below $50 \mathrm{MeV}$, on the basis of the O'Brien spectra. Outside the cascade core, the simulation data from all energies ( $10 \mathrm{GeV}$ to $1 \mathrm{TeV}$ ) and all materials (iron, aluminum, and tungsten) were well described by a common conversion factor $(1.22 \pm 0.02) \times 10^{-7}$ rem star ${ }^{-1} \mathrm{~cm}^{2}$ after dividing by the inelastic mean free path at the incident proton energy. For concrete, for which the mean free path is $49 \mathrm{~cm}$, the result is then $5.9 \times 10^{-6} \mathrm{rem} \mathrm{star}^{-1} \mathrm{~cm}^{3}$, or two thirds of Van Ginneken's value.

On this basis, one might legitimately reduce the dose equivalents quoted elsewhere in this report by a factor of 1.5 , but the continued use of the conversion factor $9 \times 10^{-6}$ rem star ${ }^{-1} \mathrm{~cm}^{3}$ is both conservative and prudent.

\subsection{Comparisons of the Tools}

The various programs for accelerator shielding have been compared among themselves at a number of different energies and also with experiment in a number of publications. These comparisons are discussed in Appendix B, where representative figures are presented. Broadly speaking, all of the tools have been used for shielding estimates at existing facilities, that is, up to beam energies of $1 \mathrm{TeV}$. It is predominately at beam energies of 300,400 and now $800 \mathrm{GeV}$ where comparisons with experimental measurements have been made. The general agreement with measurements and among calculations is good for the hadronic component.

At higher energies, measurements are not available. The codes CASIM, FLUKA, and MARS have been intercompared for hadrons at beam energies of 3,10 , and $20 \mathrm{TeV}$. The agreement among the calculations is to within a factor of two or better, adequate for shielding estimation.

For the muonic component, the comparisons are less complete. A few comparisons with measurements have been made at beam energies up to $800 \mathrm{GeV}$, but only fragmentary intercomparisons among models have been made at higher energies, largely because CASIM is the only code at present with a reasonable treatment of muon propagation and degradation. The muon calculations have additional uncertainties concerning the absolute magnitude of production of the prompt muons at $20 \mathrm{TeV}$. As described in Section 2.3, the assumptions used in CASIM lead to a considerable over-estimate of the most energetic component of both the prompt and secondary muons. We believe this provides an adequate safety margin in the estimation of dose equivalents for the muons at the SSC.

A discussion, case by case, of the accuracy of the results presented in Chapter 3 is given in Section 3.7. 


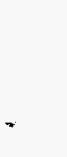




\section{RESULTS}

In this section we present the results of calculations of dose equivalents from hadrons and muons resulting from routine dumping of the beam, from the pp collisions at an interaction region, and from scraping the beam. Where it is appropriate, we estimate the shielding required to yield an annual dose equivalent of $10 \mathrm{mrem}$ or less for each type of operational loss. We also discuss the dose equivalents for accidental loss of the beam and the specific activity in ground water resulting from SSC operation.

In our calculations we have assumed the following conditions:

1. Routine beam dumps: $2 \times 10^{17}$ protons per year at an energy of $20 \mathrm{TeV}$.

2. Interaction regions: $2 \times 10^{16}$ interactions (at $40 \mathrm{TeV}$ ) per year.

3. Beam cleanup regions: $0.2 \%$ to $1.0 \%$ of the full beam at $20 \mathrm{TeV}$.

4. Accidental beam loss: $4 \times 10^{14}$ protons at $20 \mathrm{TeV}$ at any location around the $\mathrm{SSC}$ ring.

The rationale for these assumptions is discussed in greater detail in Chapter 1. Briefly, the beam-beam collisions with $4 \times 10^{14}$ protons in each beam would result in a luminosity of $10^{34} \mathrm{~cm}^{-2} \mathrm{~s}^{-1}$ at a low $\beta$ interaction region, i.e. 10 times the design luminosity. The value of $2 \times 10^{17}$ protons (at $20 \mathrm{TeV}$ ) dumped per year results from assuming 500 aborts per year at the maximum intensity of $4 \times 10^{14}$ protons. Assuming $10^{9}$ interactions per second (resulting from a luminosity of $10^{34} \mathrm{~cm}^{-2} \mathrm{~s}^{-1}$ ) for approximately 6000 hours per year yields a total of $2 \times 10^{16}$ interactions per year.

\subsection{Annual Dose Equivalent from Hadrons-Beam Dump}

During operation of the SSC, it will be necessary to abort the bearns and send them to beam dumps where the energy of the beams is absorbed. This procedure is described in some detail in the SSC Conceptual Design Report [1]. The beam dump will be designed to contain the radioactivity induced by the beam for both personnel protection on the surface near the dump and to prevent local ground water contamination (See Section 3.10.). Since the dump area will be under the immediate control of the SSC laboratory, the dump may be designed to meet these goals without impacting the land area required for the SSC site.

For completeness we show a calculation of the lateral annual dose equivalent for $2 \times 10^{17}$ protons dumped into a simulated beam dump consisting of soil of density $2.24 \mathrm{~g} \mathrm{~cm}^{-3}$. Since the effective density of the dump may easily be made to exceed this value, the results somewhat overestimate the required lateral 
shielding. Fig. 5 contains the isodose curves calculated with CASIM [26] for both soil and concrete. In Fig. 6 the maximum dose as a function of the radius (derived from Fig. 5) is shown for soil.

For comparison, we show in Fig. 6 the dose equivalent in rem vs. radius from the line of impact of the beam, calculated from the formula given by Stevenson [37]:

$$
H=6.9 \times 10^{-13} E^{0.8} \frac{e^{-(R-a) / \lambda}}{R^{2}}
$$

Here $E$ is the proton energy in $\mathrm{GeV}, R$ is the radius from the center line, $a$ is the radius of any empty space, if present, and $\lambda$ is an effective absorption length. For a given soil density $\rho$, the value of $\lambda$ may be calculated [38] from

$$
\lambda=\frac{\lambda^{\prime}}{\rho},
$$

where $\lambda^{\prime}$ is taken to be $117 \mathrm{~g} \mathrm{~cm}^{-2}$. At the larger distances the two calculations are in agreement. In the $10 \mathrm{mrem}$ region, both indicate that approximately $10 \mathrm{~m}$ of soil would be required for effective lateral shielding.

\subsection{Annual Dose Equivalent from Hadrons-Side Walls at an Inter- action Region}

The proton-proton collisions in an interaction region in the SSC produce hadrons that may interact in the side walls and roof surrounding the interaction point. The isodose curves from the interaction of these hadrons in soil of density $2.24 \mathrm{~g} \mathrm{~cm}^{-3}$ as calculated with CASIM[26] are shown in Fig. 7. The collision hall is simulated as a cylinder of radius $10 \mathrm{~m}$ and length $380 \mathrm{~m}$ (the length assumed does not influence the calculation, only the radius). In Fig. 8 the maximum dose vs. wall or roof thickness as derived from Fig. 7 is plotted for $2 \times 10^{16}$ interactions per year for soil of density $2.24 \mathrm{~g} \mathrm{~cm}^{-3}$. Also shown is a comparable calculation using PYTHIA [17] to simulate p-p collisions at $40 \mathrm{TeV}$ and FLUKA [14] to simulate the hadronic cascade in the walls. The difference between the two calculations results primarily from differences in simulating $p-p$ collisions at $40 \mathrm{TeV}$. The difference in the shielding required is about $2 \mathrm{~m}$ to reach the 10 mrem limit per year. However, it should be noted that the greater radius (as calculated by PYTHIA + FLUKA) is essentially the same as required for the beam cleanup regions (See Fig. 12). 


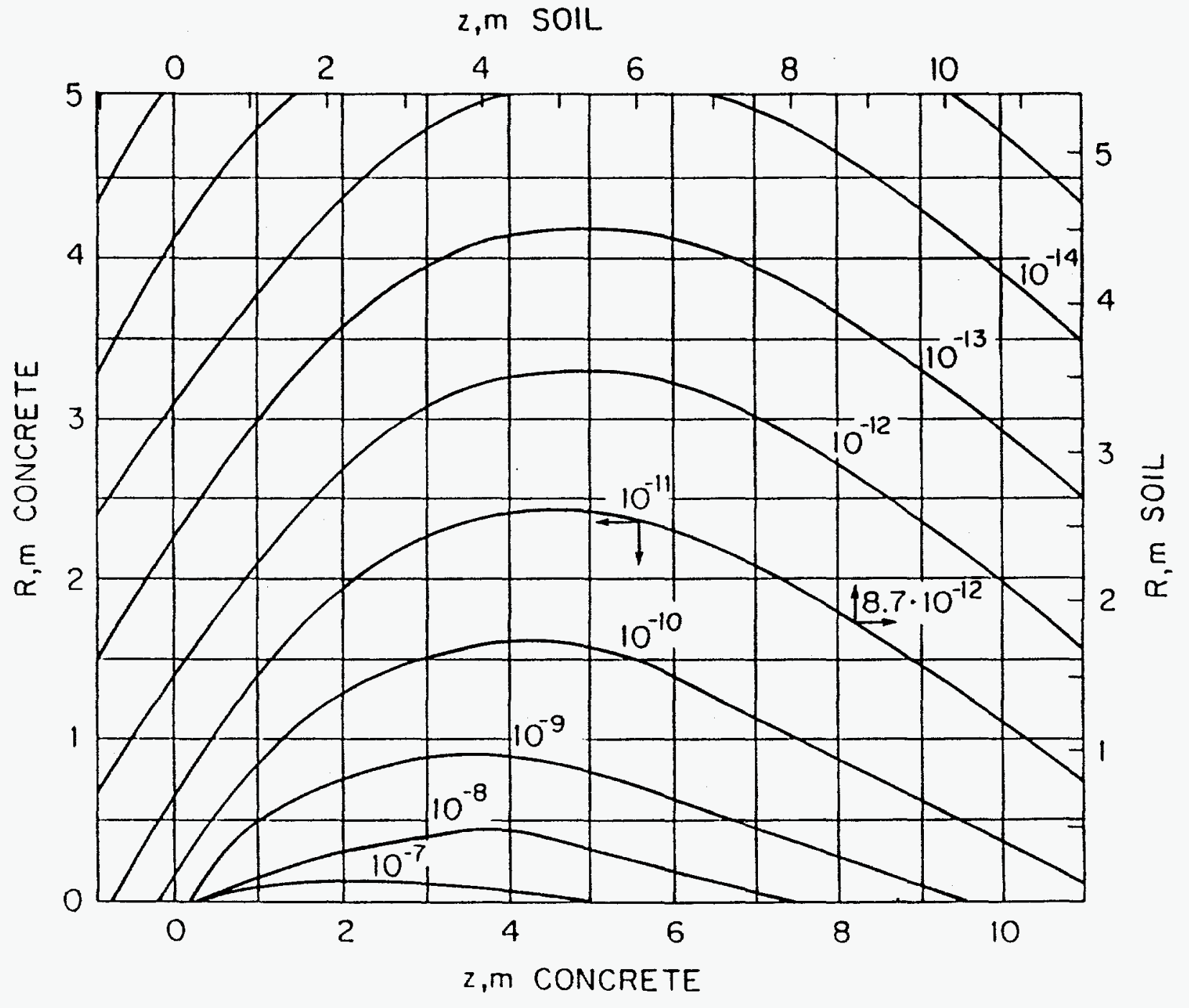

Figure 5. Contours of equal dose equivalent (in rem/incident proton) for $20 \mathrm{TeV}$ protons incident on a solid concrete or soil cylinder. The calculations are from Ref. 26. The density of concrete is $2.4 \mathrm{~g} \mathrm{~cm}^{-3}$, and soil is $2.24 \mathrm{~g} \mathrm{~cm}^{-3}$. Because the dose equivalent is proportional to the square of the density, the contours correspond to different constant doses, as indicated by the numbers with different sets of arrows pointing toward the relevant pairs of scales. 


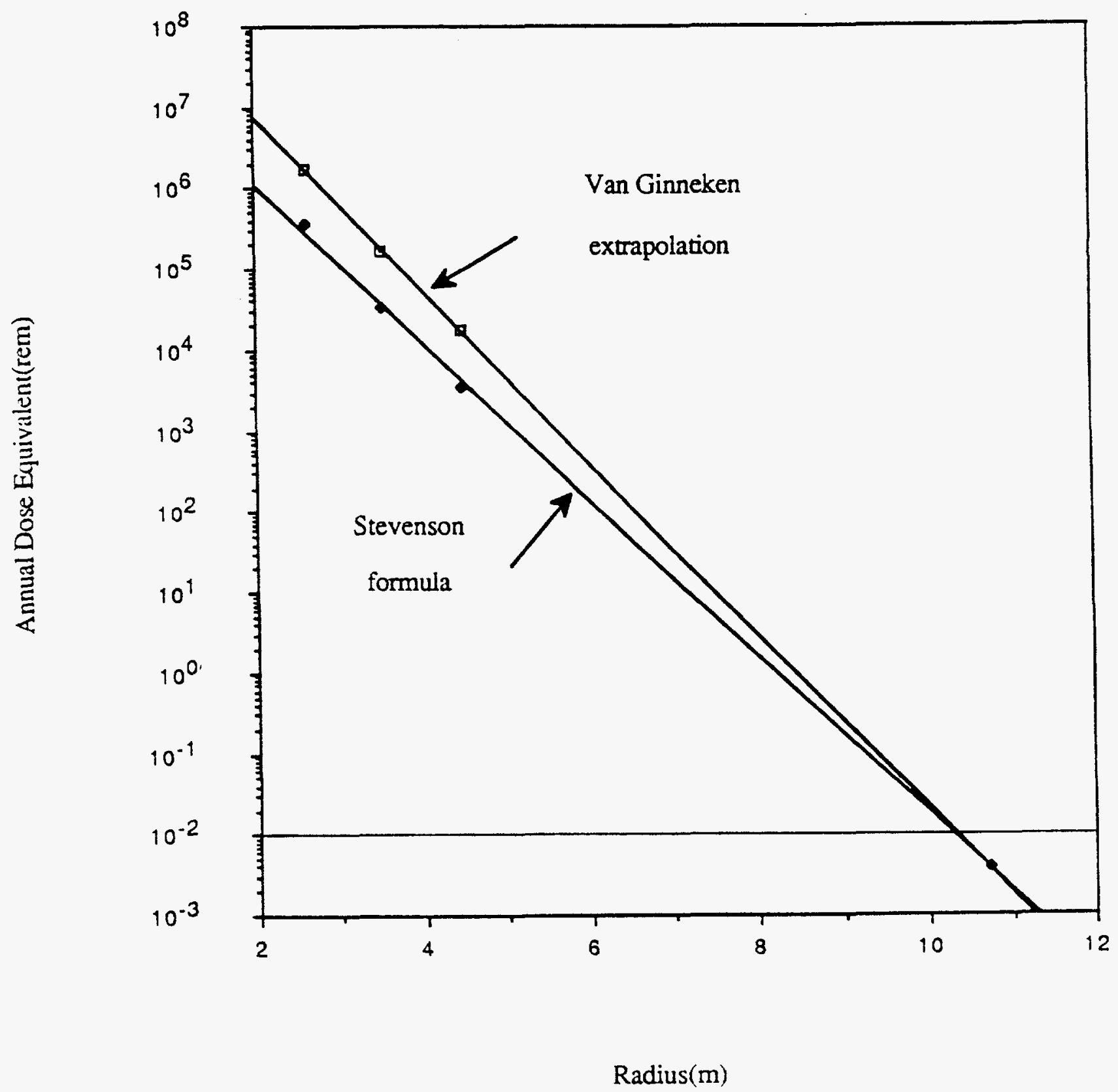

Figure 6. Annual dose equivalent in rem for $2 \times 10^{17}$ protons dumped into soil of density $2.24 \mathrm{~g} \mathrm{~cm}^{-3}$ vs. radius from the line of impact of the beam at $z \approx 6 \mathrm{~m}$. 


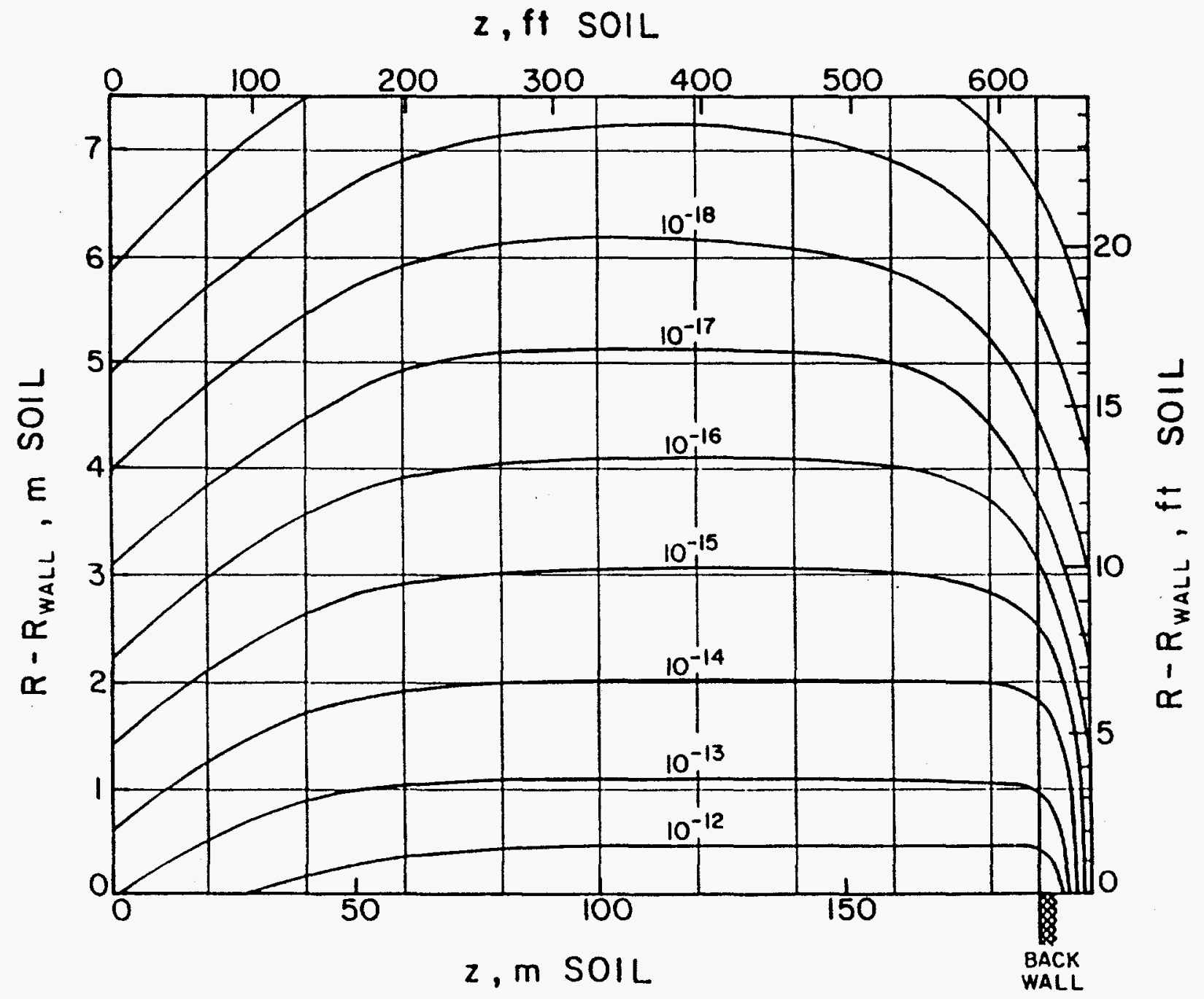

Figure 7. Contours of equal dose equivalent (in rem/inelastic collision) in the soil side wall (or roof) of a collision hall, due to colliding beams of $20 \mathrm{TeV}$ each. The calculations are from Ref. 26. Soil density is 2.24 $\mathrm{g} \mathrm{cm}^{-3} ; R_{\text {wall }}=9 \mathrm{~m}$. 
Hadron dose: IR side walls

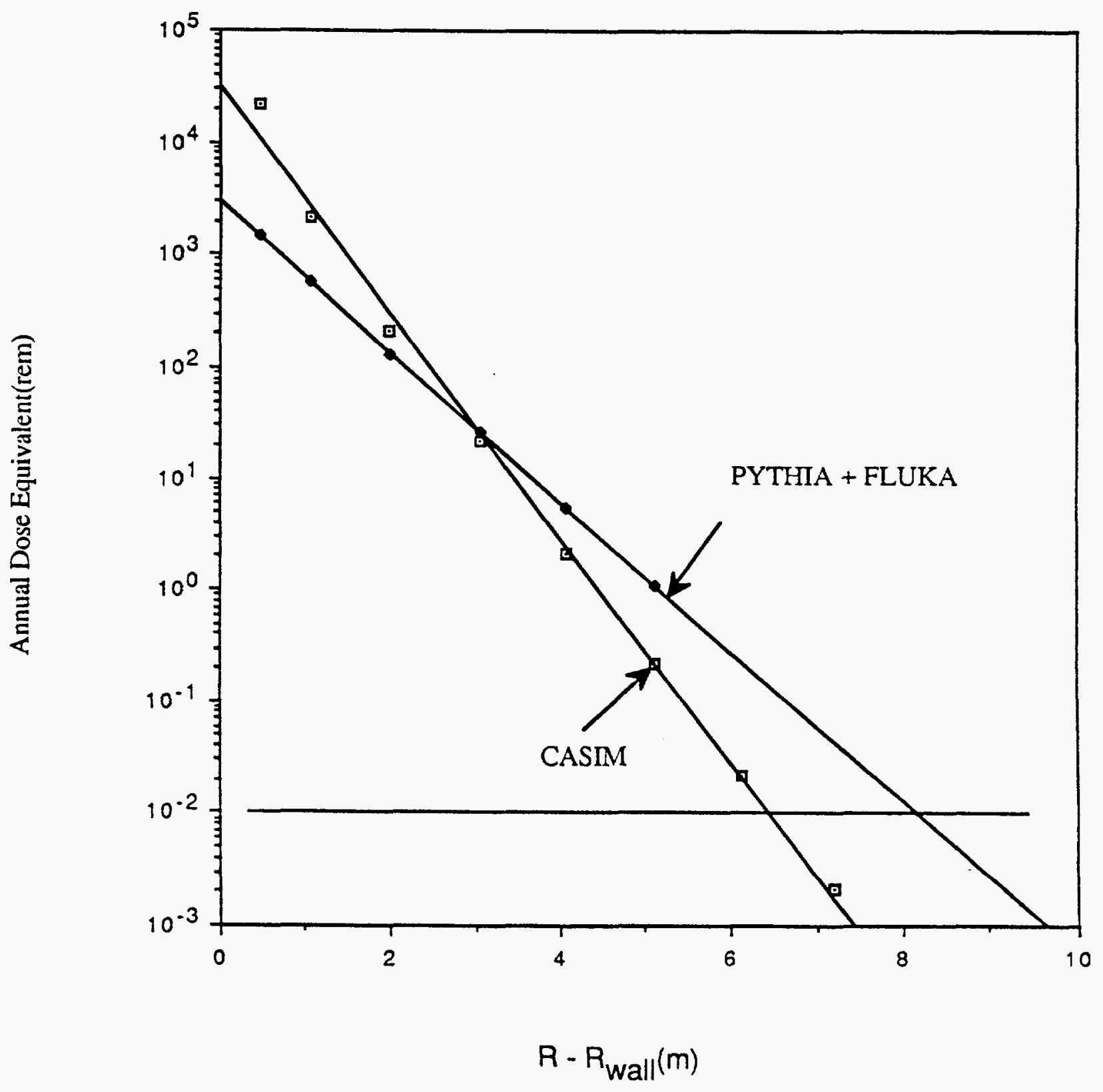

Figure 8. Dose equivalent in rem from hadrons in the side walls (or roof) of a collision hall vs. the thickness of the soil for $2 \times 10^{16}$ interactions per year of colliding $20 \mathrm{TeV}$ beams. The radius of the hall $\left(R_{\text {wall }}\right)$ is $10 \mathrm{~m}$, and the soil density is $2.24 \mathrm{~g} \mathrm{~cm}^{-3}$. 


\subsection{Annual Dose Equivalent from Hadrons-Beam Cleanup Regions}

At a small number of points around the tunnel are located special beam scrapers whose purpose is to remove the unwanted diffuse halo of particles that surrounds the dense central core. Removal of the halo improves the quality of the beam at the interaction points. The scrapers consist of stops placed very close to the beam and of sufficient length effectively to remove the unwanted protons from the periphery of the beam. For the purposes of shielding the scrapers can be modeled by the CASIM simulations [26] for the loss of protons in the beam pipe of a continuous dipole. Figures 9, 10, and 11 show the isodose contours for loss on the inside, in the middle, and on the outside of the beam pipe, respectively, where inside and outside refer to locations toward and away from the center of the accelerator. The soil density is $2.24 \mathrm{~g} \mathrm{~cm}^{-3}$. The loss location with the greatest dose at a given radius is for beam loss in the middle of the beam pipe (Fig. 10), the situation closest to that of the scrapers.

Even though the beam cleanup regions are specially designed and can be provided with auxiliary dense shielding if necessary, we give a conservative estimate by scaling the results of Fig. 10, to light soil $\left(1.8 \mathrm{~g} \mathrm{~cm}^{-3}\right)$, using the method described in Ref. 26. Fig. 12 shows the radial dependence of the annual dose equivalent in rem so derived from Fig. 10 at approximately $z=25 \mathrm{~m}$ (where the highest dose occurs). The two solid curves correspond to an average beam loss of $0.2 \%$ from scraping, equivalent to $4 \times 10^{14}$ protons per year and applicable to possible scraper units located in the arcs. The simple Stevenson formula stated earlier is seen to agree well with the CASIM numerical simulations. The dashed curve gives the dose equivalent versus radius for $1.0 \%$ beam loss $\left(2 \times 10^{15}\right.$ protons per year). The $10 \mathrm{mrem}$ lateral distance is $10 \mathrm{~m}(0.2 \%$ loss $)$ to $11 \mathrm{~m}(1.0 \%$ loss $)$ from the tunnel center in light soil. The dot-dashed curve shows that the corresponding distance is 8 to $8.7 \mathrm{~m}$ in dense soil $\left(2.24 \mathrm{~g} \mathrm{~cm}^{-3}\right)$. The latter values are applicable to the main scrapers, located in the utility straight sections where the effective dense soil conditions can be created artificially if need be.

\subsection{Dose Equivalent from Hadrons-Accidental Beam Loss}

The unanticipated loss of the full $20 \mathrm{TeV}$ beam is a very unlikely occurrence. Such a loss would likely do considerable damage to the accelerator and require months of repair time. Despite its major impact on the operation of the machine, it does not add to the radiological impact already addressed for operations. The full beam (at 3 times the design current) has $4 \times 10^{14}$ protons. Its loss at one spot is equivalent in radiation dose equivalent to the beam cleanup region dose for $0.2 \%$ loss from scraping. The solid curves in Fig. 12 thus give the dose as a 


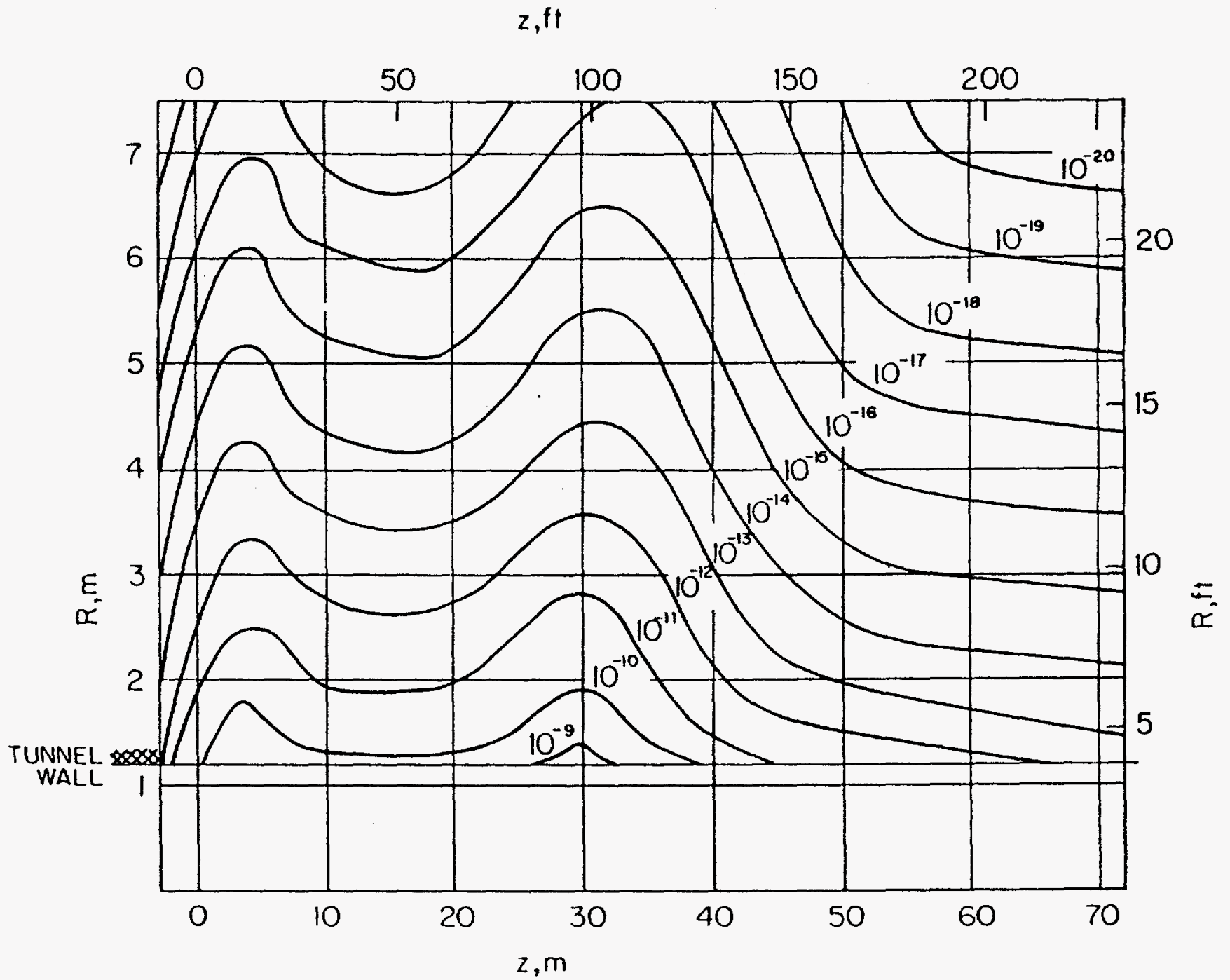

Figure 9. Contours of equal dose equivalent (in rem/interacting proton) in soil around a tunnel when $20 \mathrm{TeV}$ protons are lost on the inside (with respect to the center of the ring) of the beampipe of a continuous dipole. The calculations are from Ref. 26 . Soil density is $2.24 \mathrm{~g} \mathrm{~cm}^{-3}$. 


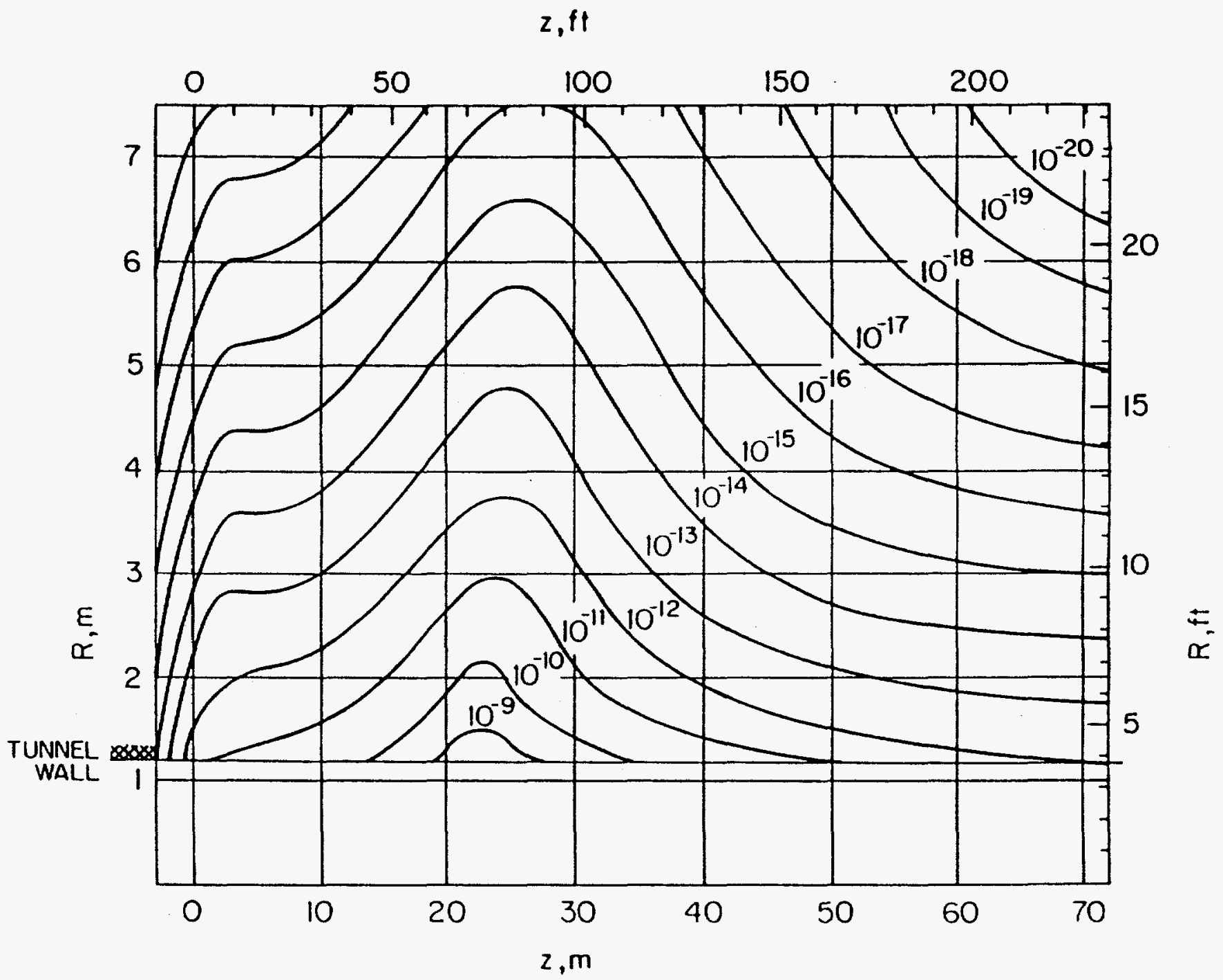

Figure 10. Contours of equal dose equivalent (in rem/interacting proton) in soil around a tunnel when $20 \mathrm{TeV}$ protons are lost in the middle of the beampipe of a continuous dipole. The calculations are from Ref. 26. Soil density is $2.24 \mathrm{~g} \mathrm{~cm}^{-3}$. 


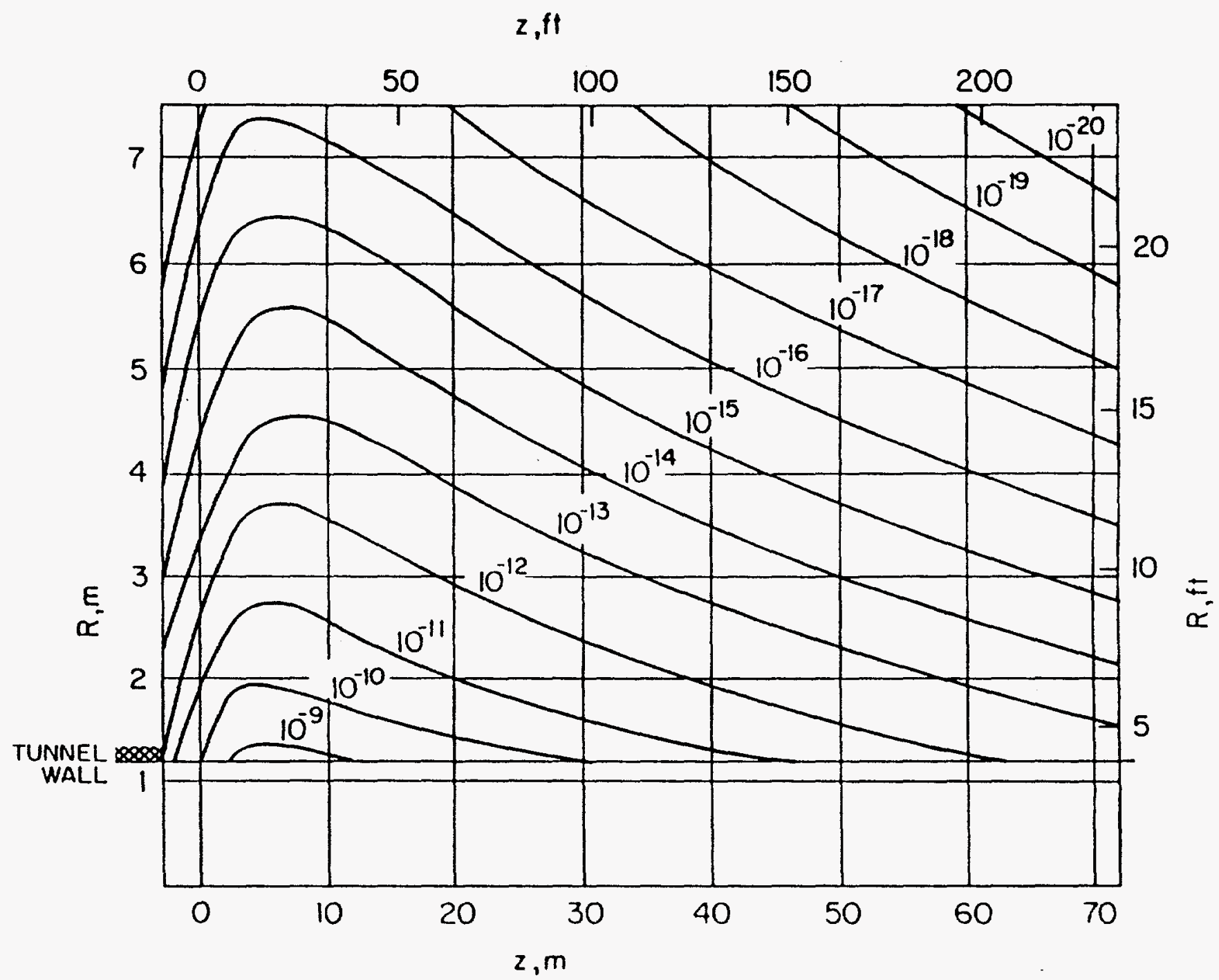

Figure 11. Contours of equal dose equivalent (in rem/interacting proton) in soil around a tunnel when $20 \mathrm{TeV}$ protons are lost on the outside (with respect to center of the ring) of the beampipe of a continuous dipole. The calculations are from Ref. 26 . Soil density is $2.24 \mathrm{~g} \mathrm{~cm}^{-3}$. 


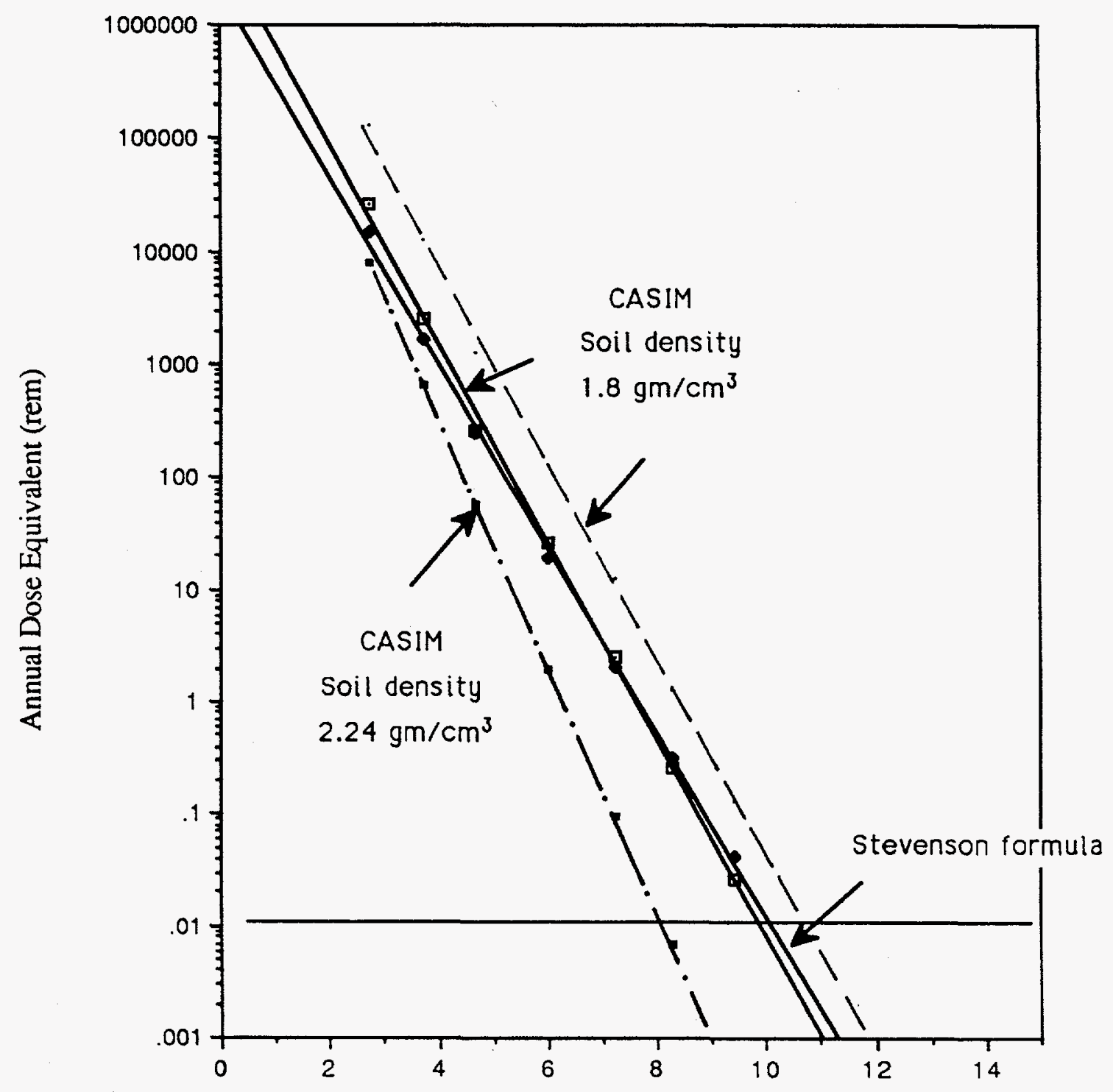

Radius (m)

Figure 12. Dose equivalent in rem vs. lateral radius from tunnel center $25 \mathrm{~m}$ downstream from beam scraper. Solid and dashed curves are for $0.2 \%$ and $1.0 \%$ beam loss, respectively, with soil density $1.8 \mathrm{~g} \mathrm{~cm}^{-3}$. Dot-dashed curve is for $0.2 \%$ loss and density $2.24 \mathrm{~g} \mathrm{~cm}^{-3}$. 
function of lateral radius. The requirement that the center of the tunnel be at least 35 feet $(10.7 \mathrm{~m})$ below ground from the various operational considerations is seen to reduce the one-time hadron dose equivalent from accidental beam loss to the level of $10 \mathrm{mrem}$ or below, even in light soil.

\subsection{Annual Dose Equivalent from Muons-Beam Dump}

Muons will be produced by the mechanisms previously described when the proton beam is brought to the beam dump. The isodose curves for $20 \mathrm{TeV}$ protons incident on solid soil as calculated with CASIM [26] are shown in Fig. 13. Because a substantial fraction of the highest energy muons result from prompt production, the use of the actual composition of the dump in a simulation would not substantially alter our conclusions. In Fig. 14 is plotted the annual dose equivalent in rem vs. the distance from the dump in kilometers for soil of density $2.24 \mathrm{~g} \mathrm{~cm}^{-3}$ for $R=0$. Although the SSC may in fact be located in soil of lower density, by adding a moderate amount of high density material (such as iron or rock) along the path of the muons downstream of the dump, the effective density could be raised to this value. Hence we have assumed this soil density to determine the required distance downstream of the dump. To reach $10 \mathrm{mrem}$ per year a distance of approximately $5.2 \mathrm{~km}$ is be required.

\subsection{Annual Dose Equivalent from Muons-Interaction Regions}

There are two regions of interest to determine the muon dose from p-p collisions-along the tangent to the ring at the collision point and transverse to the beam axis in the collision hall region. In Fig. 15 we show the isodose curves as calculated with CASIM [26] along the tangent to the ring and radially out from it. Many of the muons from the p-p collisions will be produced at small angles with respect to the colliding beam axis and therefore will pass through the magnet structure. This will tend to disperse the muons, reducing the dose at long distances. This effect has not been taken into account in our calculations. Furthermore, as noted in Chapter 2, the prompt muon production model used in CASIM very likely overestimates the muon flux at high muon momentum for colliding beams at $E_{\text {c.m. }}=40 \mathrm{TeV}$. For these two reasons we have chosen to use an effective soil density of $2.24 \mathrm{~g} \mathrm{~cm}^{-3}$, rather than $1.8 \mathrm{~g} \mathrm{~cm}^{-3}$, to determine the length of the vector tangent to the ring at the collision point from which the land requirements are derived. In Fig. 10 we show the annual dose equivalent in rem vs. distance in kilometers along the tangent in the plane of the machine for soil of density $2.24 \mathrm{~g} \mathrm{~cm}^{-3}$. A distance of approximately $4.3 \mathrm{~km}$ is required to attain the $10 \mathrm{mrem}$ per year limit. If the soil density were $1.8 \mathrm{~g} \mathrm{~cm}^{-3}$, the distance would be approximately $5.3 \mathrm{~km}$. 
z,km LIGHT SOIL

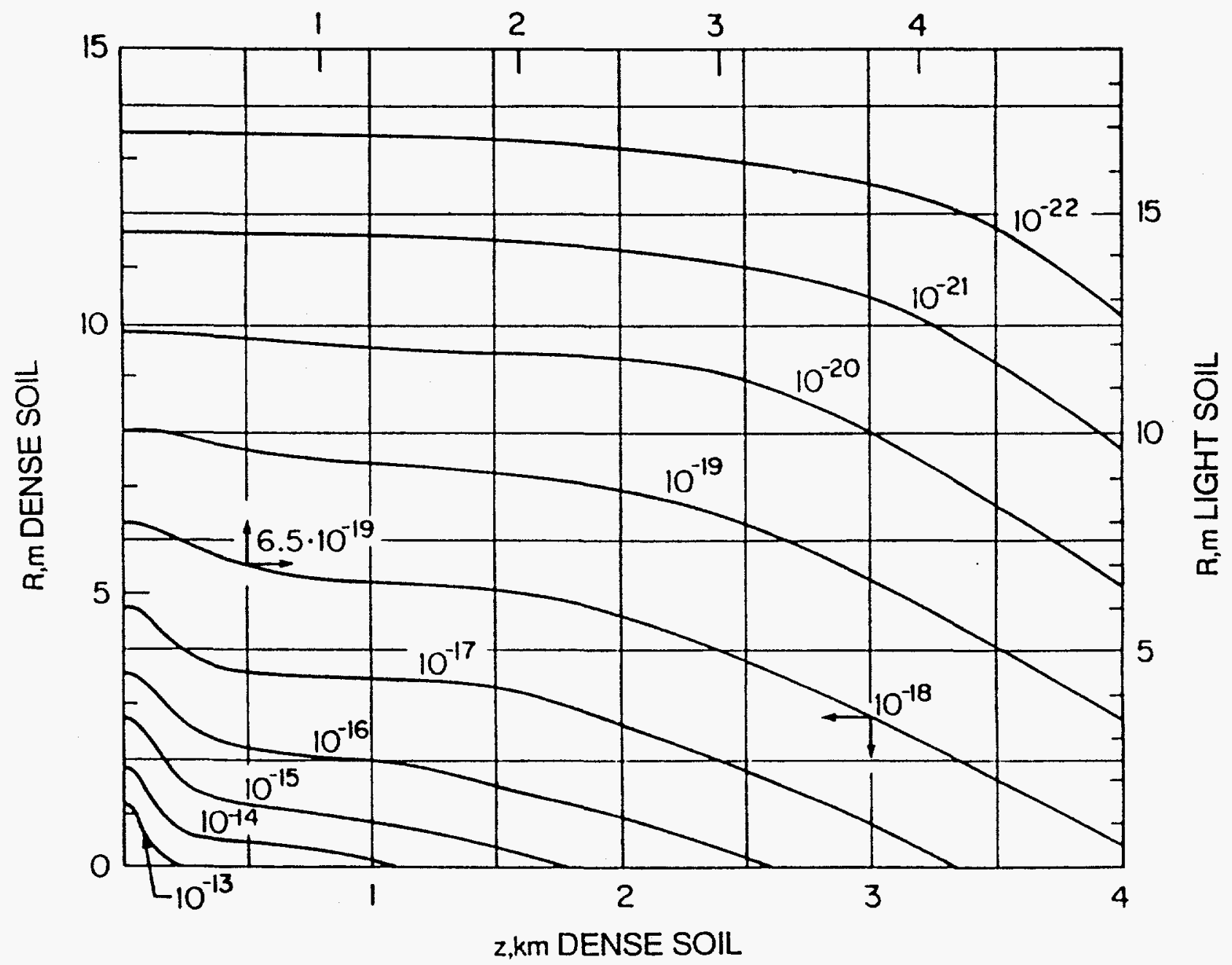

Figure 13. Contours of equal dose equivalent (in rem/incident proton) from muons for a beam of $20 \mathrm{TeV}$ protons incident on a solid soil cylinder. The calculations are from Ref. 26. Scales for dense and light soils of densities 2.24 and $1.8 \mathrm{~g} \mathrm{~cm}^{-3}$, respectively, are shown. Because the dose equivalent is proportional to the square of the density, the contours represent different values of dose for dense and light soil, as indicated by the numbers with different arrows pointing to the different pairs of scales. 


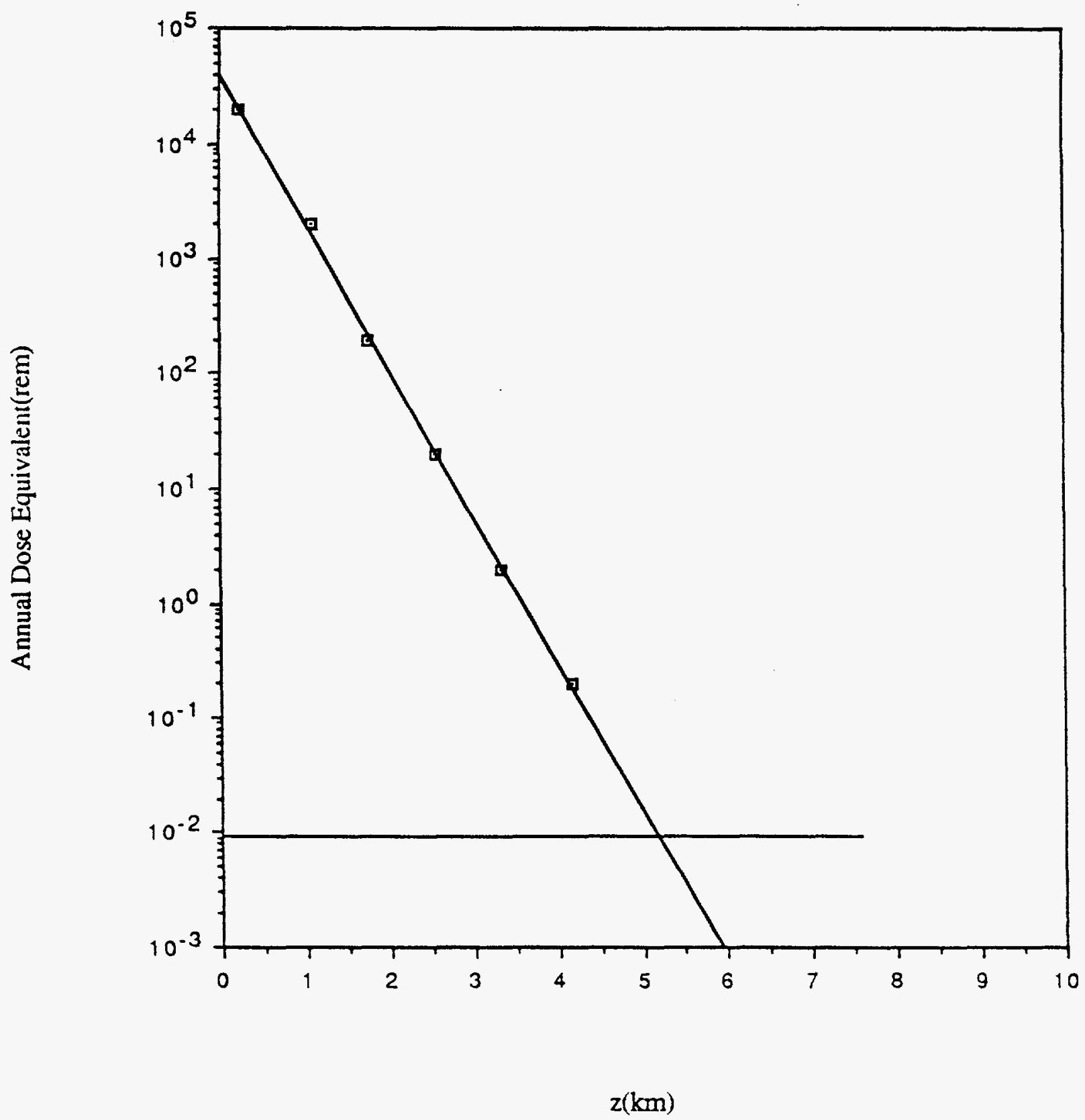

Figure 14. Annual dose equivalent in rem from muons resulting from dumping $2 \times 10^{17}$ protons at $20 \mathrm{TeV}$ in soil vs. the distance downstream of the dump. Soil density is $2.24 \mathrm{~g} \mathrm{~cm}^{-3}$. 
z,km LIGHT SOIL

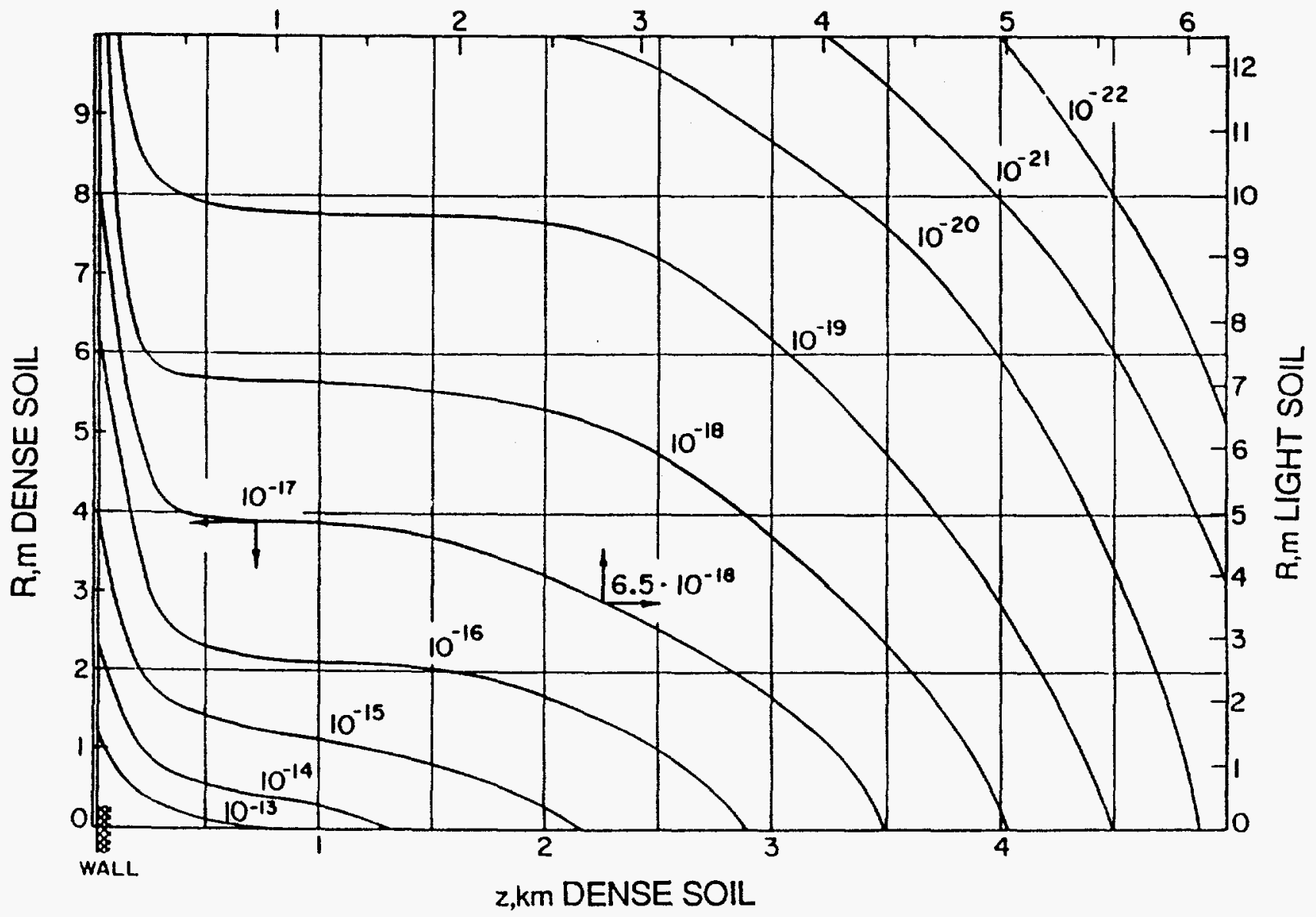

Figure 15. Contours of equal dose equivalent (in rem/inelastic collision) in soil from muons from colliding beams at $20 \mathrm{TeV}$ each along the axis of the colliding beams. The calculations are from Ref. 26. Scales for dense and light soils of densities 2.24 and $1.8 \mathrm{~g} \mathrm{~cm}^{-3}$, respectively, are shown. Because the dose equivalent is proportional to the square of the density, the contours represent different values of dose for dense and light soil, as indicated by the numbers with different arrows pointing to the different pairs of scales. 


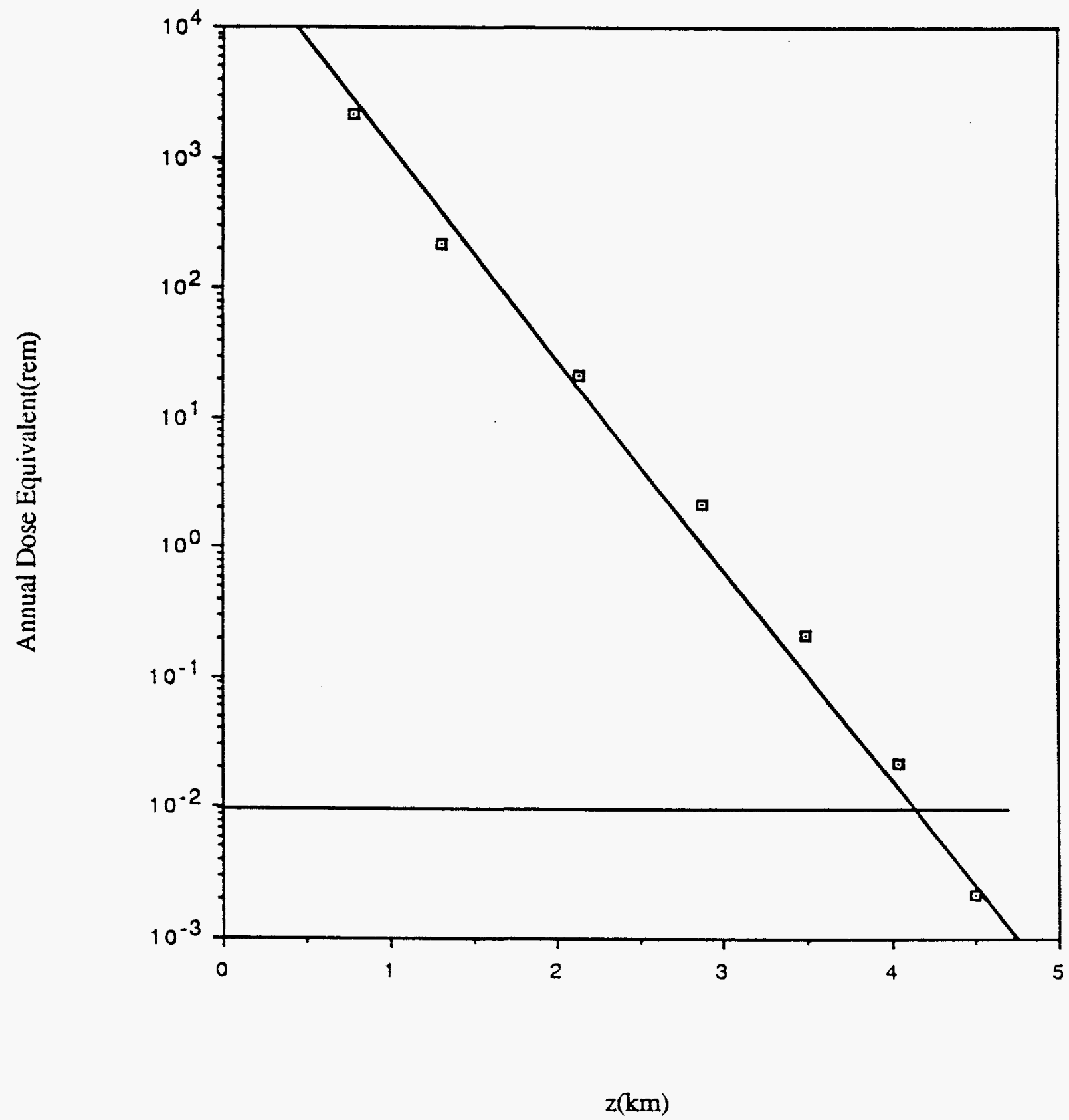

Figure 16. Annual dose equivalent in rem from muons produced in $2 \times 10^{16}$ interactions of $20 \mathrm{TeV}$ beams at an interaction region vs. the distance along the beam axis in soil. Soil density is $2.24 \mathrm{~g} \mathrm{~cm}^{-3}$. 
The isodose curves for muons in the sidewalls or roof at an intersection region as calculated with CASIM [26] are shown in Fig. 17. For this figure a decay length of $10 \mathrm{~m}$ was assumed. The annual dose equivalent in rem from $2 \times 10^{16}$ interactions per year vs. the thickness of the sidewall or roof shield $\left(R-R_{\text {wall }}\right)$ is shown in Fig. 18 for soil of density $2.24 \mathrm{~g} \mathrm{~cm}^{-3}$. A preliminary calculation using PYTHIA to simulate pion and kaon production, which yield muons by decay in a hall of $10 \mathrm{~m}$ radius, and a simple range-energy relationship to determine the effective muon dose, is also shown in Fig. 18[37]. The two calculations disagree slightly (by about $2 \mathrm{~m}$ ) in the amount of soil shielding required to reach a $10 \mathrm{mrem}$ level. Since local shielding around the beamline (or concrete in the walls or roof) or shielding from a detector located at the intersection point can be assumed to be in place, one may substantially reduce the annual dose at a given thickness. At large angles, the dose from prompt muon production is small compared to that from pion and kaon decay. Shielding provided either by a detector or specially inserted at the IR can easily reduce the muon flux by one or two orders of magnitude.

\subsection{Annual Dose Equivalent from Muons-Beam Cleanup Regions}

The scraping of the beam at selected positions around the ring will create energetic muons as well as the hadrons discussed in Sect. 3.3. As described in Chapter 1, the total muon flux contains components from "prompt" muon production, from the decays of pions and kaons before they are absorbed in the soil surrounding the tunnel, and from hadron or photon production in the cascade process in the soil.

The processes described above are simulated by CASIM [26], as discussed briefly in Chapter 2. Calculations have been made for interaction of the beam on the inside (with respect to the ring center), the outside, and the center of the beam pipe in a continuous magnet. The results are qualitatively similar for the three cases; the interactions on the inside of the beam pipe give the largest ranges and so are chosen for dose estimation. Figs. 19, 20, and 21 display the isodose contours for three different quadrants of a soil shield surrounding the tunnel. The $z$ direction is defined by the tangent to the ring at the location of the scraper. The radial direction is perpendicular to that tangent line. For a given dose, the largest range along the tangent occurs in the plane of the machine, on the outside at $R=0$ (Fig. 21). The doses equivalent in rem versus tangential distance $z$ in kilometers for a soil density of $1.8 \mathrm{~g} \mathrm{~cm}^{-3}$ are shown in Fig. 22 for a beam loss of $0.2 \%\left(4 \times 10^{14}\right.$ protons $)$ and $1.0 \%\left(2 \times 10^{15}\right.$ protons $)$ per year. Also shown is a $0.2 \%$ loss line for dense soil $\left(2.24 \mathrm{~g} \mathrm{~cm}^{-3}\right)$. In light soil the $10 \mathrm{mrem}$ per year dose equivalent point is at a tangent sector length of $2.3 \mathrm{~km}$ for $0.2 \%$ beam 


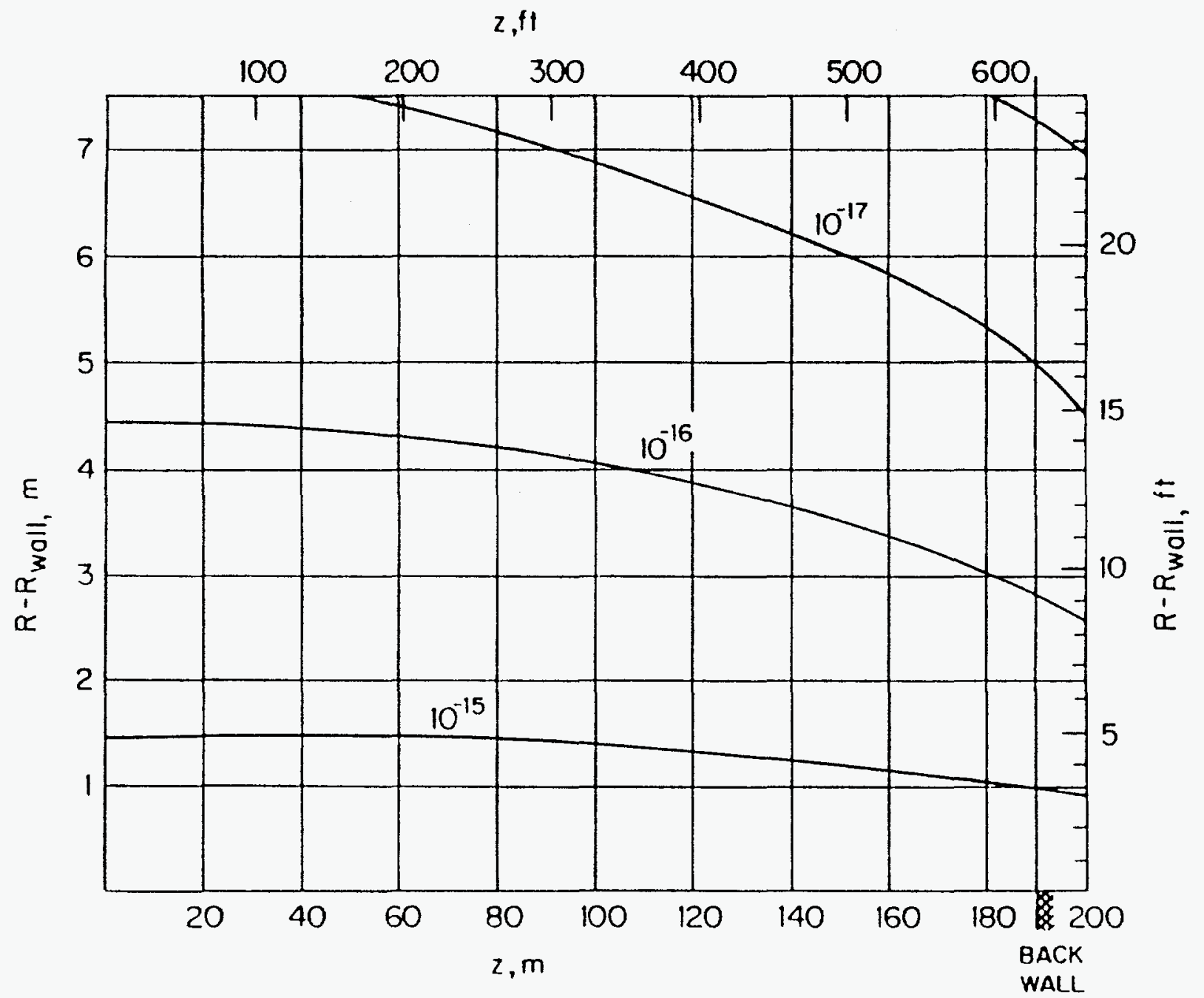

Figure 17. Contours of cqual dose equivalent (in rcm/inclastic collision) in a soil side wall (or roof) of a collision hall for colliding beams of 20 $\mathrm{TeV}$ each. The radius of the collision hall is $10 \mathrm{~m}$. The calculations are from Ref. 26. Soil density is $2.24 \mathrm{~g} \mathrm{~cm}^{-3}$. 


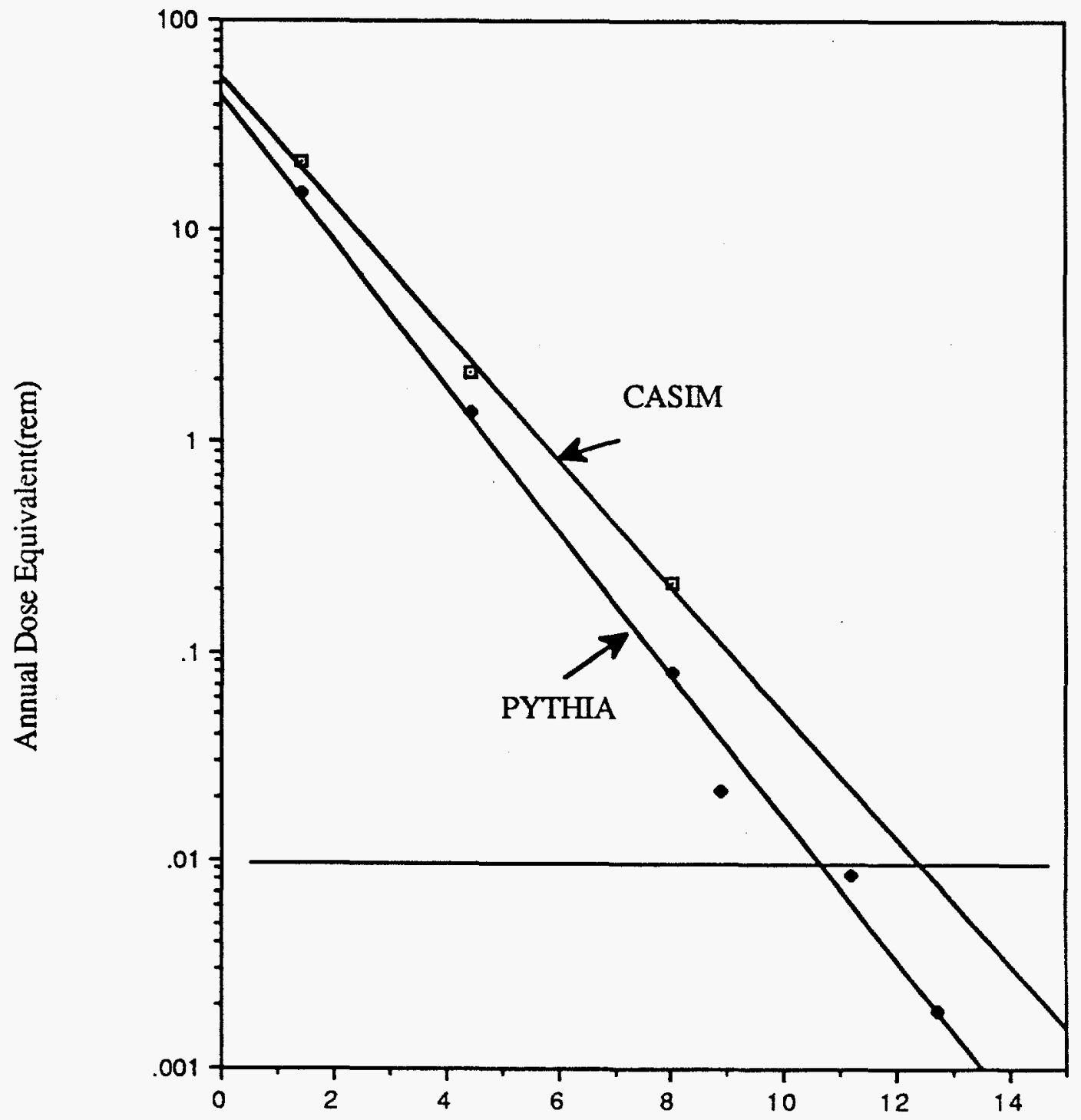

$\mathrm{R}-\mathrm{R}($ wall $)(\mathrm{m})$

Figure 18. Annual dose equivalent in rem from muons produced by $2 \times 10^{16}$ interactions per year of colliding beams of $20 \mathrm{TeV}$ each in the side walls (or roof) of collision hall vs. the thickness of the soil shicld. Soil density is $2.24 \mathrm{~g} \mathrm{~cm}^{-3} ; R_{\text {wall }}=10 \mathrm{~m}$. 


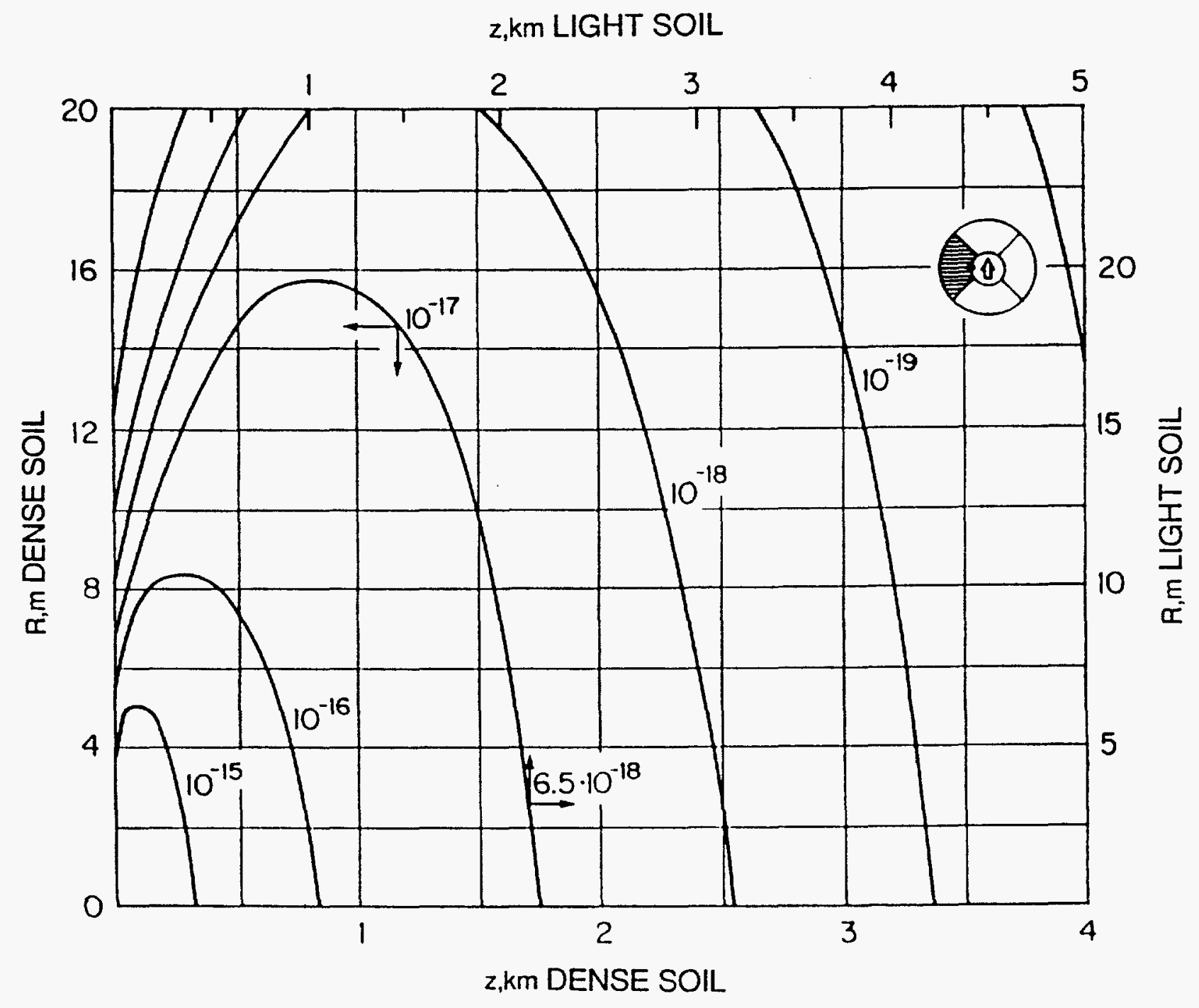

Figure 19. Contours of equal dose equivalent (in rem/interacting proton) in the inside quadrant (with respect to the ring) of soil shield around the tunnel from muons produced by the interactions of $20 \mathrm{TeV}$ protons on the inside of the beampipe of a continuous magnet in the tunnel. The calculations are from Ref. 26. Scales for dense and light soils of densities 2.24 and $1.8 \mathrm{~g} \mathrm{~cm}^{-3}$, respectively, are shown. Because the dose equivalent is proportional to the square of the density, the contours represent different values of dose for dense and light soil, as indicated by the numbers with different arrows pointing to the different pairs of scales. 


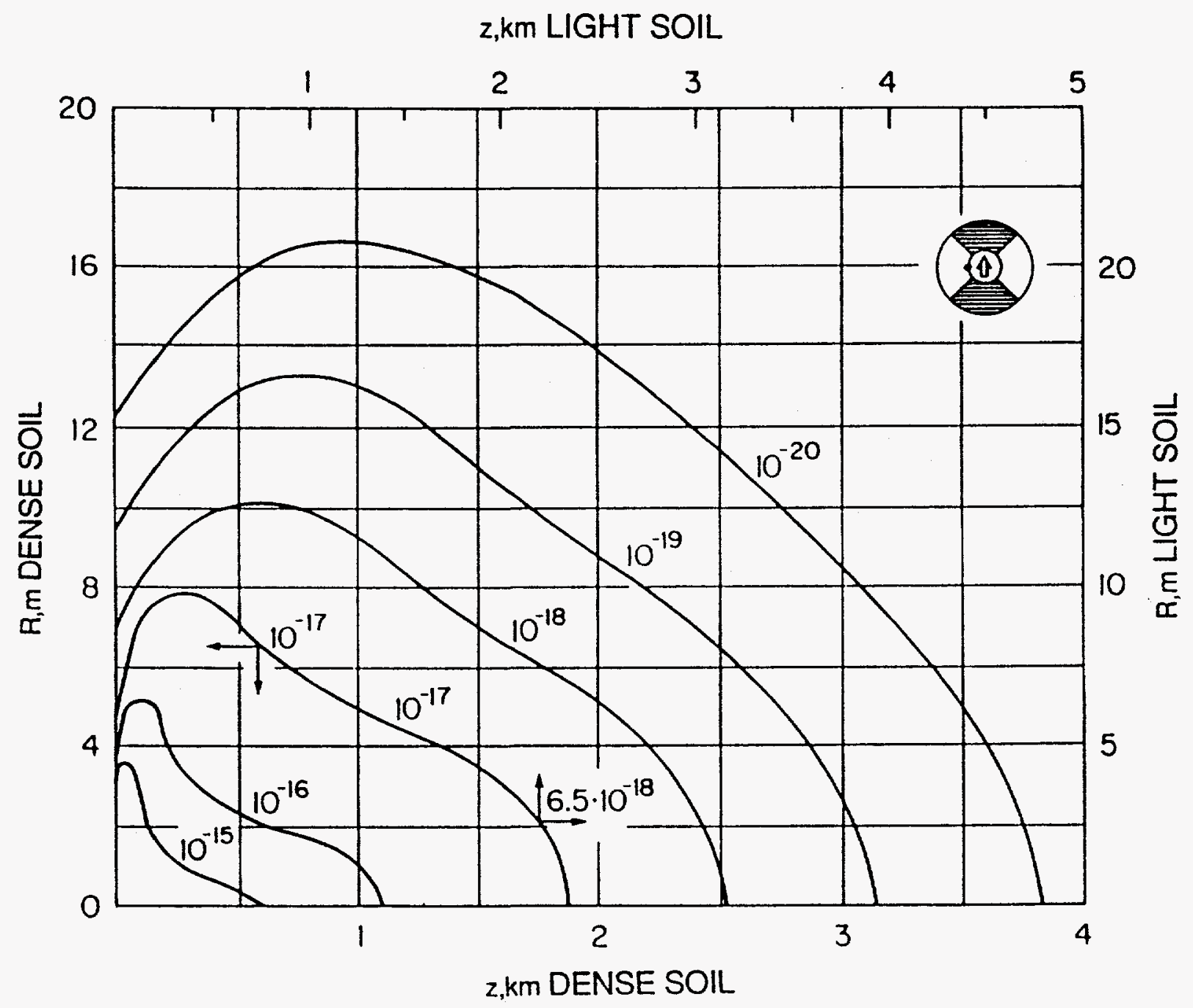

Figure 20. Contours of equal dose equivalent (in rem/interacting proton) in the top (bottom) quadrant (with respect to the ring) of soil shield around the tunnel from muons produced by the interactions of $20 \mathrm{TeV}$ protons on the inside of the beam pipe of a continuous magnet in the tunnel. The calculations are from Ref. 26. Scales for dense and light soils of densities 2.24 and $1.8 \mathrm{~g} \mathrm{~cm}^{-3}$, respectively, are shown. Because the dose equivalent is proportional to the square of the density, the contours represent different values of dose for dense and light soil, as indicated by the numbers with different arrows pointing to the different pairs of scales. 


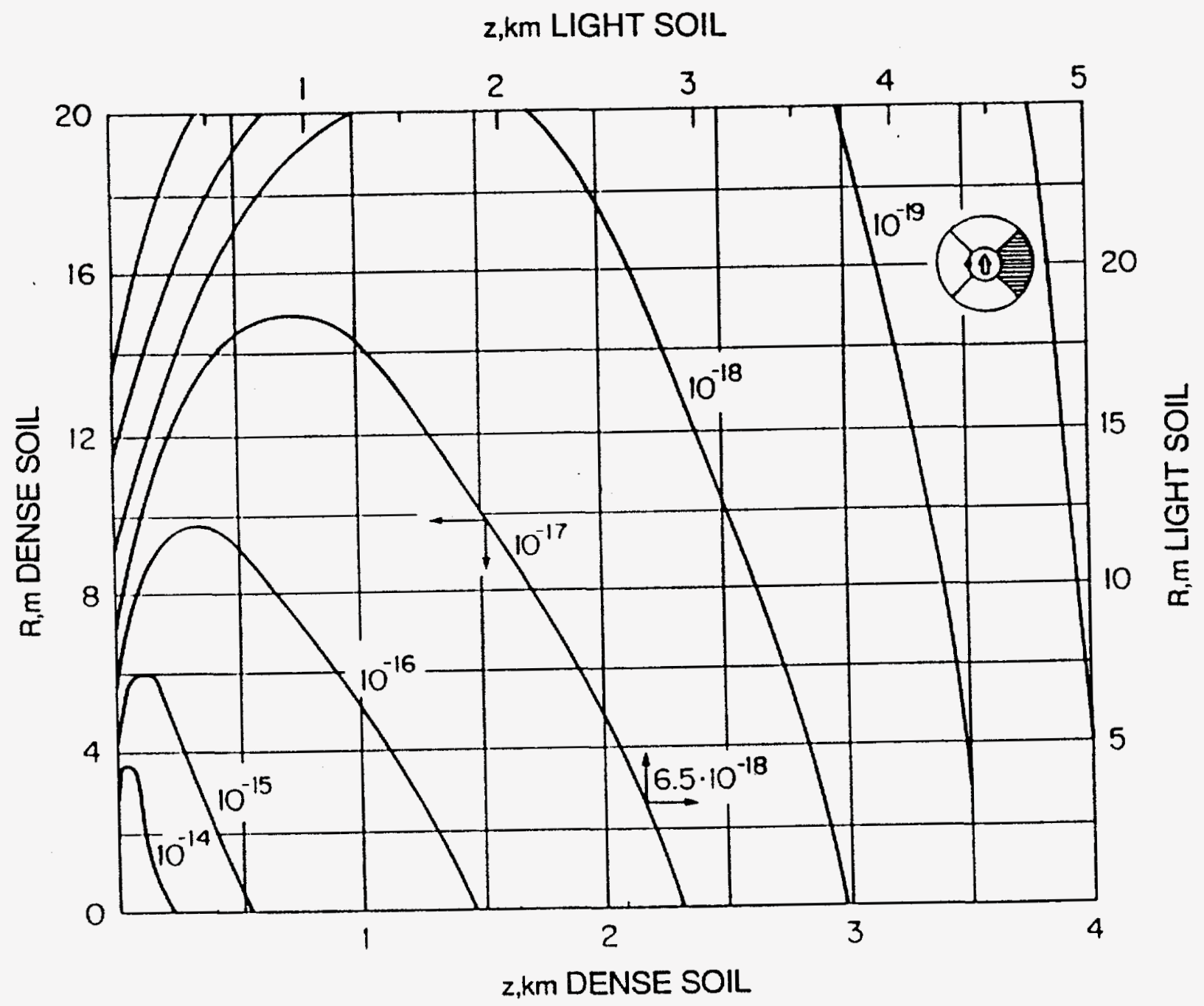

Figure 21. Contours of equal dose equivalent (in rem/interacting proton) in the outside quadrant (with respect to the ring) of soil shield around the tunnel from muons produced by the interactions of $20 \mathrm{TeV}$ protons on the inside of the beampipe of a continuous magnet in the tunnel. The calculations are from Ref. 26. Scales for dense and light soils of densities 2.24 and $1.8 \mathrm{~g} \mathrm{~cm}^{-3}$, respectively, are shown.Because the dose equivalent is proportional to the square of the density, the contours represent different values of dose for dense and light soil, as indicated by the numbers with different arrows pointing to the different pairs of scales. 


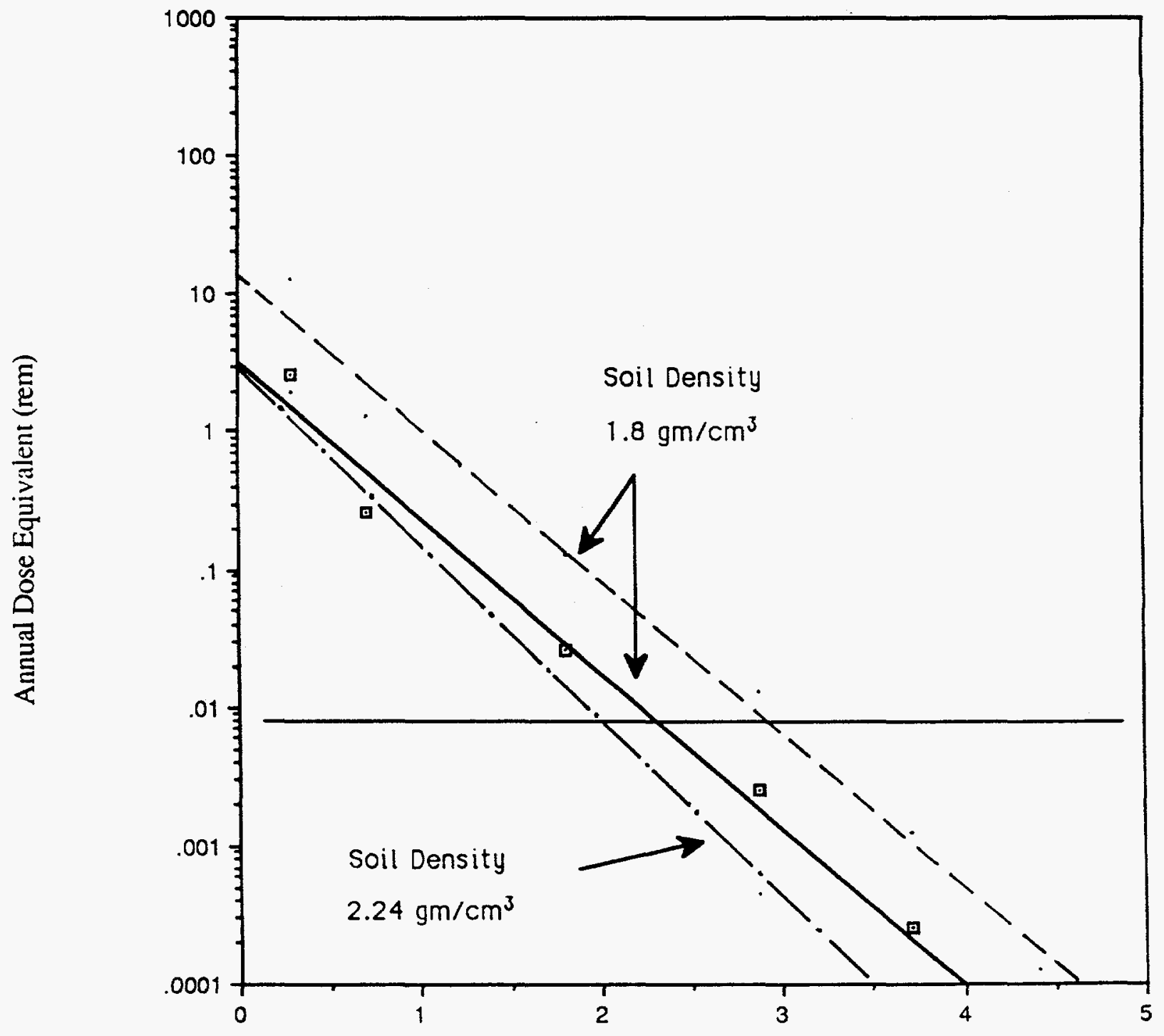

$\mathrm{z}(\mathrm{km})$

Figure 22. Dose equivalent in rem from muons produced by scraper beam loss vs. the distance along a tangent vector at the point of loss. Solid (dashed) lines are for $0.2 \%(1.0 \%)$ beam loss with soil density $1.8 \mathrm{~g} \mathrm{~cm}^{-3}$. The dot-dashed line is for $0.2 \%$ loss and density $2.24 \mathrm{~g} \mathrm{~cm}^{-3}$. 
loss and $2.9 \mathrm{~km}$ for $1.0 \%$. In dense soil, the corresponding length for $0.2 \%$ loss is $1.9 \mathrm{~km}$.

\subsection{Dose Equivalent from Muons-Accidental Beam Loss}

An unintentional loss of the full beam at some point around the ring will cause a localized hadronic cascade, as described in Section 3.4, but also a muon vector of activity in the soil along the tangent at the point of loss. The loss of $4 \times 10^{14}$ protons ( 3 times design current) corresponds to the $0.2 \%$ case for the beam cleanup regions. We take the worse case of light soil downstream from the point of loss. The solid line in Fig. 22 then gives the dose equivalent in rem as a function of distance $z$ along the tangent in kilometers.

\subsection{Comments on the Accuracy of the Calculations}

In this section we briefly comment on the accuracy of the results described above. In addition, we suggest improvements to the tools for estimating radiation dosage that could be implemented in the near future to improve the reliability of the shielding calculations. Although the estimates of radiation dosage can always be improved, the shielding dimensions determined in this report with the existing computational tools are prudent and safe. There is no reason to believe that the SSC shielding dimensions will significantly increase as the result of future improvements to the tools for estimating radiation dosage.

\section{(a) Hadron Dose-Beam Dump}

In the simplest terms, the beam dumps for the SSC may be made so as to guarantee personnel safety in the vicinity of the dump and to eliminate any question regarding local ground water contamination by making a conservative design. Such a design has no impact on the land area required for the SSC and a negligible cost impact. In this sense the present reliability of radiation dosage calculations for beam dumps is simply not an issue. Nevertheless, there is excellent agreement among the available tools (see Section 3.2) as to the lateral shielding required for a beam dump. There is poorer agreement among the tools for the required longitudinal shielding but the horizontal dimension, deep underground, is not critical. The present dump design assures that there is no ground water hazard (see Sect. 3.10); the final design will incorporate the latest information in a conservative manner.

(b) Hadron Dose-IR Side Walls and Roof

At present the best simulation of the particle yields from p-p collisions at 40 $\mathrm{TeV}$ is provided by ISAJET or PYTHIA. The production model used in CASIM is 
not as suitable. In this sense, the best estimate of the shielding required would result from combining the particle production model of ISAJET or PYTHIA with the hadronic cascade simulation of FLUKA or CASIM. So far only the combination of PYTHIA and FLUKA has been used. This indicates that the shielding required for hadrons produced in the $\mathrm{p}-\mathrm{p}$ collisions is about the same as that required to shield against an accidental beam loss in the IR. Furthermore, the amount of shielding located at an interaction region will be under the control of the SSC laboratory and can be increased if necessary.

(c) Hadron Dose-Beam Cleanup Regions and Accidental Loss

There is very good agreement between CASIM, FLUKA-based calculations and experiment on the amount of lateral shielding required in the case of beam stops-see Chapter 2 and Appendix B. We conservatively estimate that the uncertainty in the amount of lateral shielding required to obtain an dose equivalent of 10 mrem or less for the beam cleanup regions in the SSC tunnel is less than $10 \%$, approximately $2-3$ feet of soil of density $1.8 \mathrm{~g} \mathrm{~cm}^{-3}$. The specification of a minimum depth of $30 \mathrm{ft}$ to the top of the tunnel all around the ring for soil of density $1.8 \mathrm{~g} \mathrm{~cm}^{-3}$ (see Chapter 4 ) should therefore be a safe and reasonable value.

\section{(d) Muon Doses-Fixed Targets}

Discussion of the reliability of the muon dose calculations for protons on fixed targets can be separated into pieces: a model for prompt muon production; a model for pion and kaon production, from which muons are produced by decays and secondary interactions; a model for photon production, the photons then produce muon pairs by interaction in material; and a model of muon transport through material. At present our estimates of muon doses are based on CASIM calculations. As noted in Section 2.3, prompt muon production as estimated by CASIM is greater than that predicted by ISAJET, particularly for muons with high energy. On the other hand, there are large theoretical uncertainties in the model used by ISAJET to calculate the production of the charm, bottom and top mesons that yield the prompt muons after decay. Since the amount of shielding required to attain an annual dose equivalent of $10 \mathrm{mrem}$ depends significantly on the prompt muon production rate, it is prudent to use the results of CASIM to determine the shielding dimensions at this time. Clearly a calculation of the muon dose using ISAJET or PYTHIA to simulate the initial p-nucleus collision and CASIM to simulate the hadronic cascade and muon transport should be done and might indicate that less shielding would be required.

In brief, the calculations based on CASIM for the muon doses for beam dumps, beam cleanup regions, and accidental loss may be viewed as conservative 
as far as estimates of shielding are concerned.

(e) Muon Dose-IR Regions

Comments similar to those above also apply to using CASIM to simulate 40 $\mathrm{TeV}$ p-p collisions. The prompt muons are more numerous and more energetic in CASIM than in the ISAJET calculations. The overall charged hadron multiplicity in the CASIM simulation is lower than ISAJET or PYTHIA by about a factor of three at $40 \mathrm{TeV}$. Qualitatively, the effect of a higher prompt muon rate, a harder spectrum and a lower charged hadron multiplicity will be to increase the yield of high energy muons in the forward direction and therefore increase the required distance along the tangent. For the reasons already cited, it is prudent to use the CASIM calculations to estimate the required shielding distances at this time. Calculations of the muon dose using ISAJET and CASIM, to simulate hadronic cascades and muon transport, should be done.

There is perhaps some uncertainty about the flux of low energy muons from the decay of pions and kaons produced at large angles. See Fig. 18. It is these muons that are relevant to shielding in the roof area of a collision hall, if the collision hall is near the surface of the earth. Since the land area above an IR region will be part of the SSC laboratory, the uncertainties in the calculation of the muon flux at large angles do not influence the required land area for the SSC.

\section{(f) Recommendations for future study}

From the above discussion, it is clear that joining the production models of ISAJET and/or PYTHIA with the hadronic cascade models of CASIM and/or FLUKA and the muon transport of CASIM should be done. Even if this is accomplished, considerable uncertainties will remain regarding the true muon yield in $40 \mathrm{TeV}$ p-p collisions and, to a lesser extent, from $20 \mathrm{TeV}$ protons on a stationary target because the physics is unexplored or poorly known. Given these uncertainties, one must choose conservative values for the shielding dimensions required to reduce the muon dose to an acceptable level.

\subsection{Radiological Considerations for Ground Water}

The question of a possible radiological impact on water supplies due to accelerator operations has two facets. The first has an impact on land acquisition and use for any site. This is the question of the possibility of contamination of a water supply, such as a well, in proximity to the accelerator through localized production of radioactivity, by accidental loss of the beam. The second is site specific, depending on the geological and hydrological characteristics of each site. 
This is the question of the possibility of producing radionuclides in soil surrounding the accelerator, which might then migrate to a ground water supply even at some distance from the accelerator.

(a) Activity produced in a nearby well from accidental loss of beam

The primary shield of the tunnel extends for at least 30 feet surrounding the tunnel. Extending another 120 feet horizontally for a total of 150 feet on either side of the tunnel is a restricted zone in which shared use of the sub-surface region will not be permitted, except by specific authorization (Ref. 2, p. 44). Since the actual site may have wells and other penetrations already existing in the restricted zone (and in the future, penetrations there may be proposed), it is desirable to have a framework concerning radiological hazards in the vicinity of the tunnel, in particular the possible contamination of a nearby well. We therefore present a model of the specific activity expected from the localized loss of the full beam ( 3 times the design current, or $4 \times 10^{14}$ protons) in the tunnel, with the production of radionuclides in the soil surrounding the tunnel. The model, developed in detail in Appendix $\mathrm{C}$, contains a number of simplifying assumptions, but contains the essentials. The details of each actual situation will have to be examined, case by case. In the absence of a site, the model provides guidelines and a mode of description.

The relevant radionuclides for ground water contamination are ${ }^{3} \mathrm{H}$ and ${ }^{22} \mathrm{Na}$. The properties of these nuclides are given in Table 3.10-1. The activity of nuclide $i$ is $A_{i}=\lambda_{i} S_{i} N_{s}$, where $N_{s}$ is the total number of stars produced.

Table 3.10-1

Properties of ${ }^{3} \mathrm{H}$ and ${ }^{22} \mathrm{Na}$

\begin{tabular}{lcc}
\hline \hline Property & ${ }^{3} \mathrm{H}$ & ${ }^{22} \mathrm{Na}$ \\
\hline$\tau_{1 / 2}(\mathrm{~s})$ & $3.9 \times 10^{8}$ & $8.2 \times 10^{7}$ \\
$\lambda \equiv \ln 2 / \tau_{1 / 2}\left(\mathrm{~s}^{-1}\right)$ & $1.78 \times 10^{-9}$ & $8.45 \times 10^{-9}$ \\
$S($ atoms $/$ star $)[8]$ & 0.075 & 0.020 \\
Leaching Factor [42] & $\sim 1.0$ & $\leq 0.1$ \\
\hline
\end{tabular}

Other nuclides such as ${ }^{11} \mathrm{C},{ }^{7} \mathrm{Be},{ }^{14} \mathrm{C}$, have either too short or too long lifetimes, negligible leaching factors or negligible production rates and hence do not contribute significant activity in water reaching a well.

Fig. 23 shows the longitudinally integrated star density per proton for an accidental loss of the beam at some point around the tunnel. It can be seen 


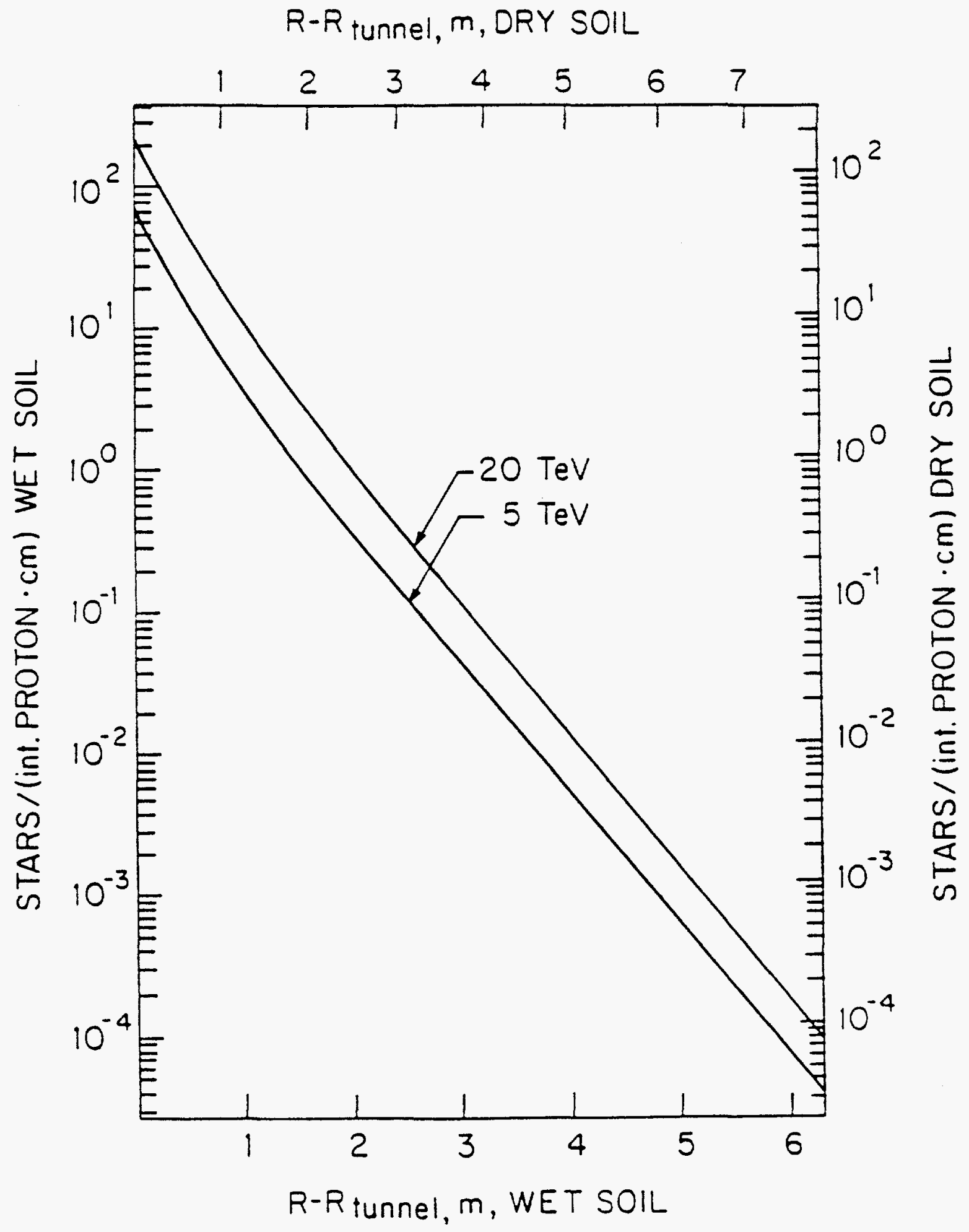

Figure 23. Longitudinally integrated star density times the circumference of a circle of radius $R$ (in stars $\mathrm{cm}^{-1}$ per interacting proton) in a soil shield around a 1.2 meter radius tunnel for 5 and $20 \mathrm{TeV}$ protons interacting on the outside (with respect to the ring) of the beam pipe of a continuous dipole inside the tunnel [26]. Soil density is $2.24 \mathrm{~g} \mathrm{~cm}^{-3}$. 
that the exponential falloff with radius has a characteristic length of the order of $0.5 \mathrm{~m}$.

The bulk of the radioactivity is therefore localized within the first meter outside the tunnel. It is also localized longitudinally within $10-20 \mathrm{~m}$. It is shown in Appendix C that Fig. 23 gives a total number of stars,

$$
N_{s}=2.5 \times 10^{18}
$$

if $4 \times 10^{14}$ protons at $20 \mathrm{TeV}$ are lost. With the parameters of Table 3.10-1, the total activities of ${ }^{3} \mathrm{H}$ and ${ }^{22} \mathrm{Na}$ potentially available to migrate to a nearby water supply are

$$
\begin{aligned}
A\left({ }^{3} \mathrm{H}\right) & =9.1 \times 10^{9} \mathrm{pCi}\left(3.4 \times 10^{8} \mathrm{~Bq}\right) \\
A\left({ }^{22} \mathrm{Na}\right) & =1.2 \times 10^{9} \mathrm{pCi}\left(4.3 \times 10^{7} \mathrm{~Bq}\right)
\end{aligned}
$$

The ${ }^{22} \mathrm{Na}$ value contains a leaching factor of $0.1[42]$.

The model of the nearby well is summarized by Fig. 39 of Appendix C. The standard hydrological model of a shallow well drawing on a cylindrically symmetric aquifer is used. Contrary to reasonable expectation, the tunnel is assumed to be below the water table and directly in the aquifer being drawn on by the well. This assumption must surely be incorrect for much of the tunnel, but may hold for portions. In any event, placing the tunnel and the hypothesized volume of radioactivity in the aquifer will over-estimate the amount of radioactivity in the well water. The assumption is thus a conservative one.

Straightforward considerations of drawing of water from the aquifer with a localized region of volume $4 \mathrm{~m} \times 3 \mathrm{~m} \times 20 \mathrm{~m}$ containing the residual radioactivity from loss of the beam at one location in the tunnel leads to the results shown in Fig. 24. The peak specific activity in the well water, relative to the standards found in EPA document 40CFR141 [43], are plotted as function of the distance of the well away from the localized region. The level of ${ }^{3} \mathrm{H}$ activity is well below the EPA standard for any relevant distance. The ${ }^{22} \mathrm{Na}$ activity is the governing quantity. With the assumptions of Appendix $\mathrm{C}$, the distance $r_{\mathrm{EPA}}$ beyond which the peak ${ }^{22} \mathrm{Na}$ level is below the EPA standard can be written is general form as

$$
r_{\mathrm{EPA}}=10 \frac{f}{p} \frac{I}{I_{D}} \text { meters },
$$

where $f$ is the fractional height of the active volume at the tunnel with respect to the thickness of the aquifer there, $p$ is the effective porosity of the soil, $I$ is the beam current at the time of the unanticipated loss and $I_{D}$ is the SSC design beam current. The results in Fig. 24 and for $r_{E P A}$ do not depend explicitly on 


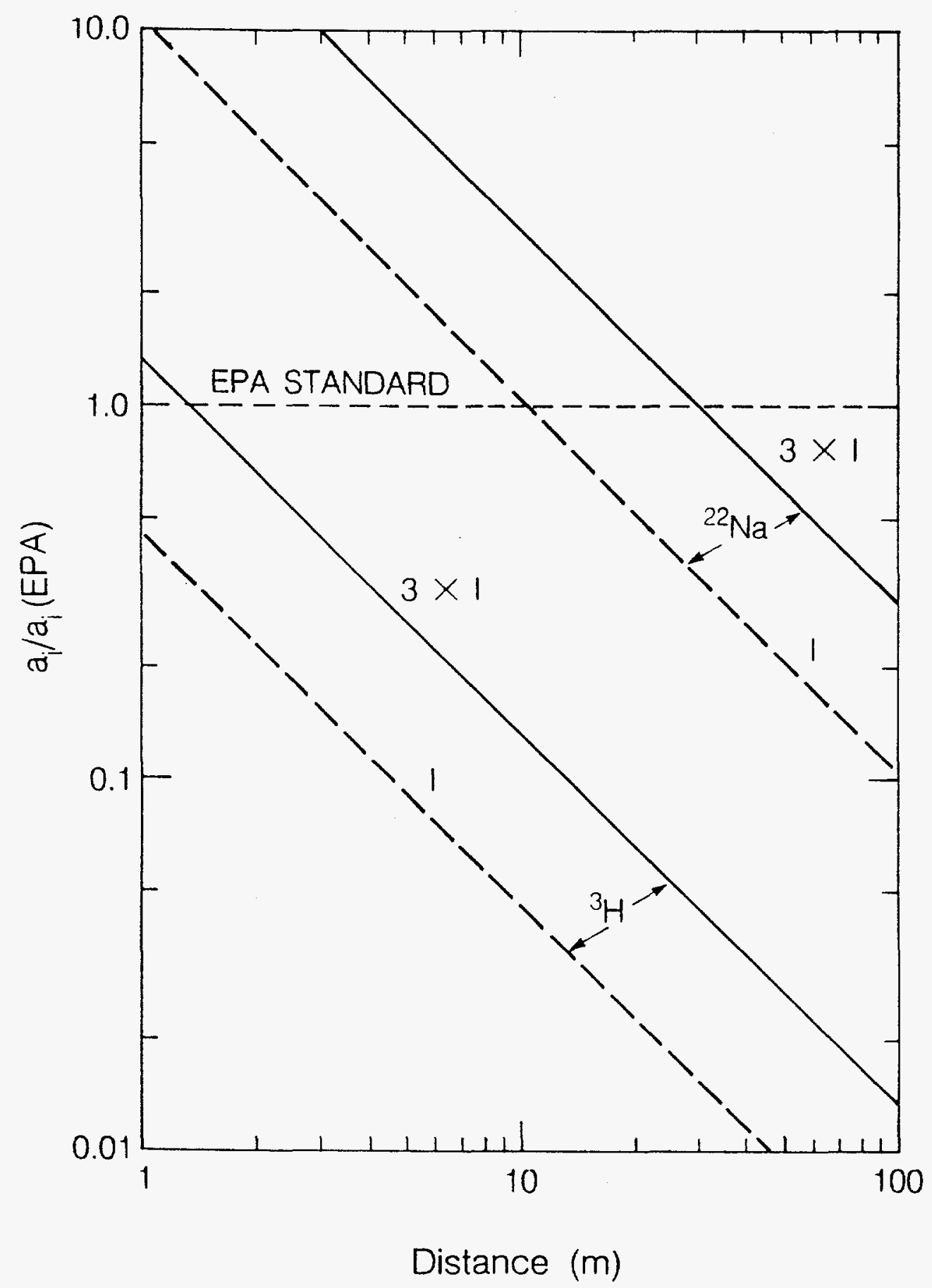

Figure 24. Peak specific activity of ${ }^{3} \mathrm{H}$ and ${ }^{22} \mathrm{Na}$ in well water expressed in units of the EPA standards, as a function of distance in meters of the well from the location of an accidental loss of the SSC beam. Two lines are shown for each nuclide, one for loss of a beam with the design current and one for 3 times design. 
the number $Q$ of gallons per day drawn from the well. Extremely high pumping rates could increase $f$ by drawing down the height of the water table, but for the standard $10^{3}$ gallons day ${ }^{-1}$, or even 10 times that, the effect is negligible.

The results of Fig. 24 and $r_{E P A}$ are for initial guidance only. They depend on a simplified model of a shallow vertical well drawing on a uniform cylindrically symmetric aquifer above a horizontal impervious layer. While this model is known to provide practical guidance in the field, the details of the specific site will take precedence. Decisions on individual wells will require investigation, case by case. The SSC Laboratory will install permanent radiation monitors in the vicinity of any wells permitted in proximity to the tunnel.

A final point about the environmental hazards to a water supply in the event of an accidental loss of the beam nearby is that there is ample time to respond and monitor the situation. It can be estimated that it will take weeks or even months for the radioactivity to reach the well. Within hours of an accidental spill of the beam, Environmental Safety teams from the laboratory will be monitoring the region intensively, to assure the integrity of water supplies in the area.

(b) Beam disposal/ground water protection

The primary locale for production of radioactive nuclides during routine operation of the accelerator is in the beam abort dumps where the proton beams are routinely disposed of at the end of each accelerator cycle. These two dumps, one for each proton beam of the Collider Ring, are located near the injection points, adjacent to the Injector complex, as shown in Fig. 1. Approximately $11 \%$ of the energy of the dumped beam results in production of radioactive nuclei [40]. The beam dump structures must be designed to contain this activity in a manner that precludes it from reaching the groundwater system.

The abort dump design from the Conceptual Design Report is shown in Fig. 25. The beam is first absorbed by a carbon cylinder that is 2 meters in diameter and 10 meters in length. This absorbs the thermal and mechanical shock of the beam striking it. Carbon has the further advantage of being a relatively light nucleus so that the number of possible radioactive nuclei that can be produced is minimized. Surrounding the carbon core is a concrete shell extending 8 meters beyond the core in the longitudinal direction to complete the absorption of the beam and also seal out any ground water in the vicinity. The outside of the concrete shell is sealed with waterproofing.

Making use of the CASIM calculations for carbon shown in Fig. 26, the radially integrated number of stars per $\mathrm{cm}$ along the axis of the dump at the interface between the carbon and concrete is estimated at 2 stars $\mathrm{cm}^{-1}$ per incident proton. The effective absorption length in the concrete at a distance of $10 \mathrm{~m}$ can be 


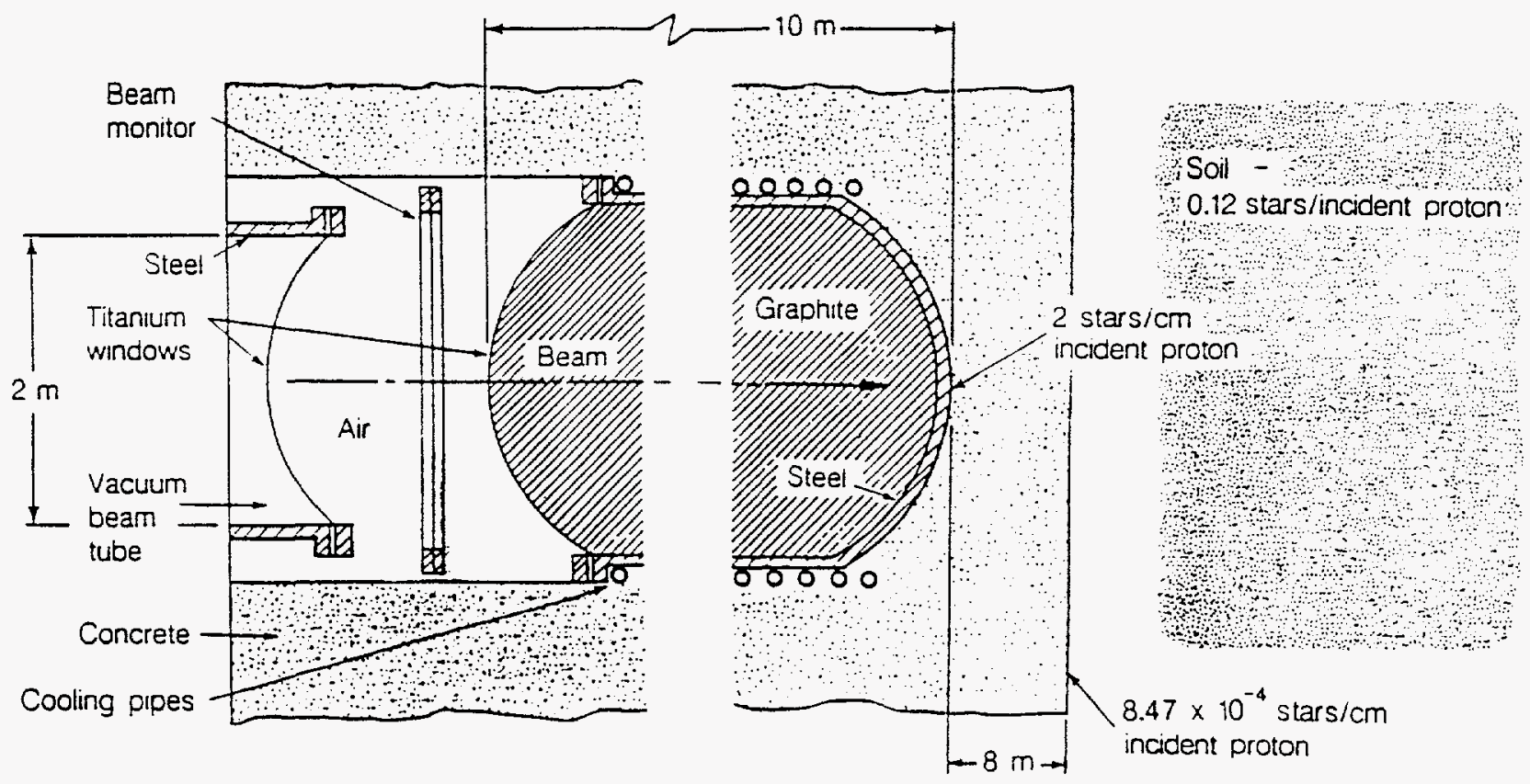

Figure 25. Collider Ring beam abort dump, longitudinal section[1]. 


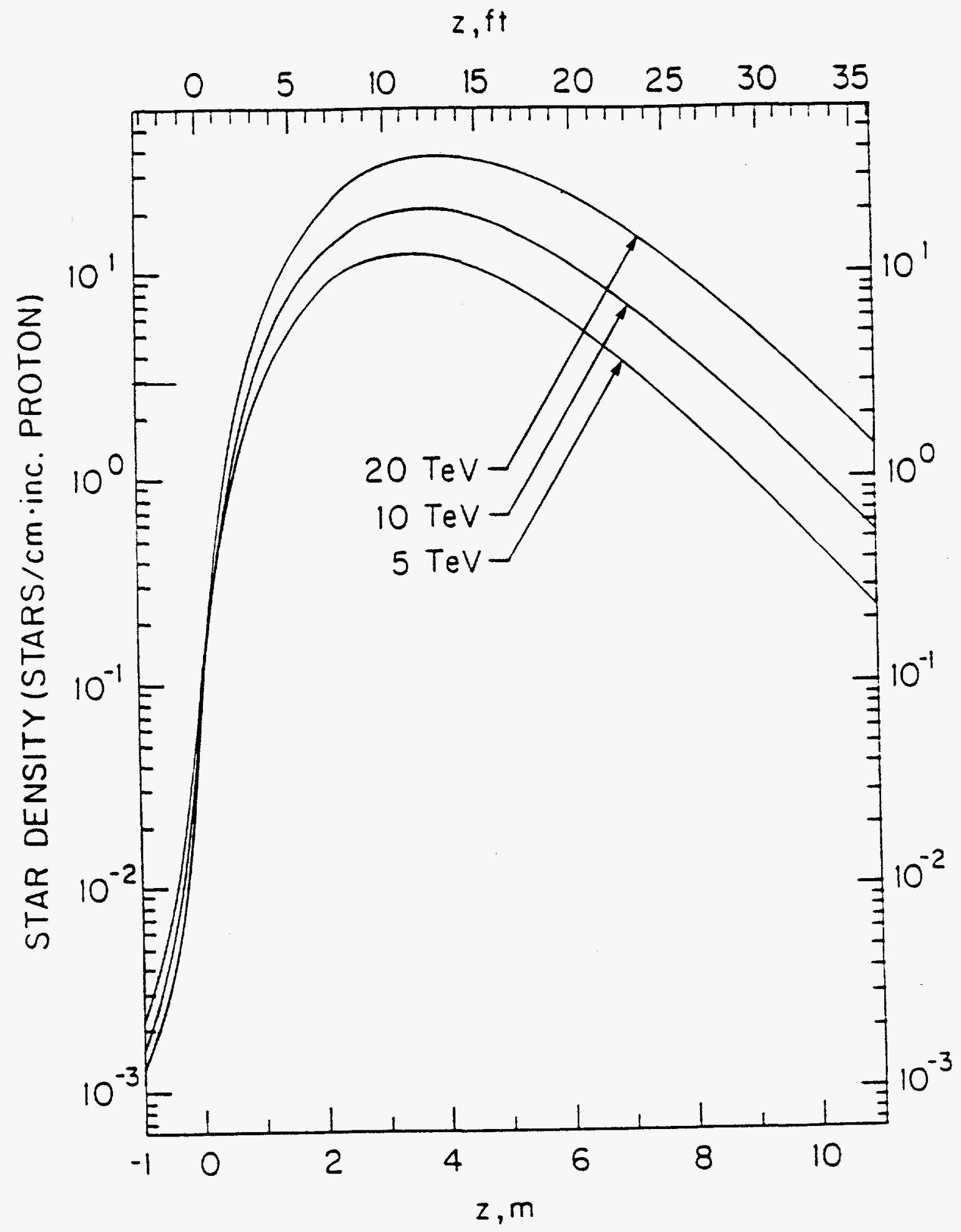

Figure 26. Radially integrated star density (in stars $\mathrm{cm}^{-1}$ per incident proton) for 5,10 , and $20 \mathrm{TeV}$ protons incident on a 12 meter long solid carbon cylinder. The calculations are from Ref. 20. 


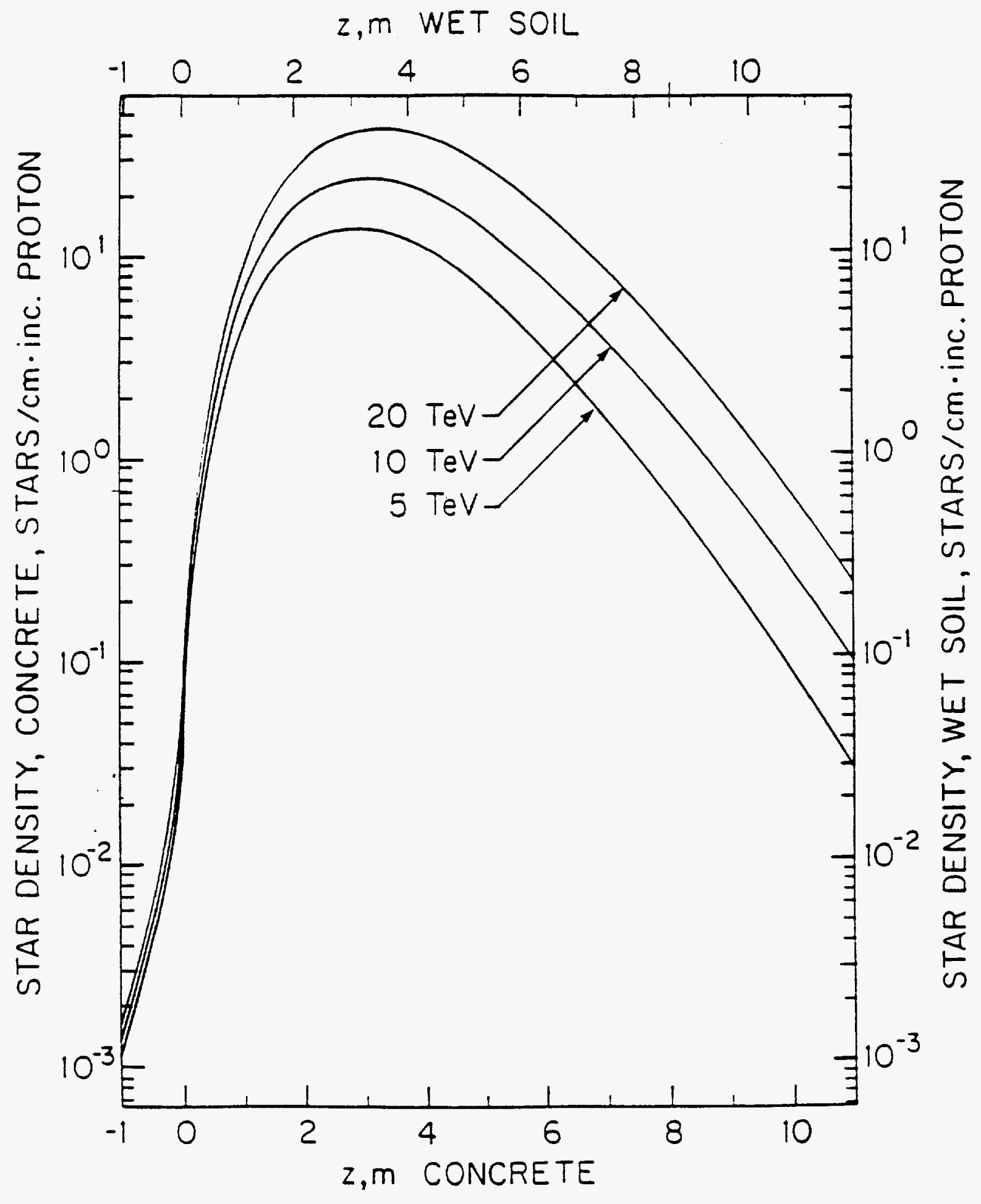

Figure 27. Radially integrated star density (in stars $\mathrm{cm}^{-1}$ per incident proton) for 5,10 and $20 \mathrm{TeV}$ protons incident on a 12 meter long solid concrete or soil cylinder [20]. Concrete (soil) density is $2.4(2.24) \mathrm{g} \mathrm{cm}^{-3}$. 
estimated from Fig. 27 to be

$$
\lambda_{\text {concrete }}=103 \mathrm{~cm} \text {. }
$$

The star density at the end of the 8 meters of concrete is thus

$$
I_{\text {concrete }}=8.5 \times 10^{-4} \mathrm{stars} \mathrm{cm}^{-1} \text { per incident proton. }
$$

Using an effective attenuation length of $137 \mathrm{~cm}$ for light soil (density $1.8 \mathrm{~g} \mathrm{~cm}^{-3}$ ) and integrating to infinity, the number of stars in unprotected soil is

$$
N_{D}=0.12 \text { stars per incident proton }
$$

(c) Absorption and transport of radioactive isotopes

To evaluate any possible impact on ground water supplies it is necessary to study the relationship between stars and resultant radioactive nuclei. Furthermore, the path these nuclei must follow to enter the ground water system must be understood. These are specific to the geology and hydrology of a particular area. However, it is possible to make an evaluation by assuming that all of the radionuclides are transported without diminution due to decay, and without allowing for the tortuous journey that substantially reduces their activity enroute.

With 500 aborts per year of $4 \times 10^{14}$ protons each, the rate of protons dumped is $2 \times 10^{17}$ protons $\mathrm{yr}^{-1}$ or an average of $6.3 \times 10^{9}$ protons $^{-1}$. With 0.12 stars per proton, the average rate of star production in the neighborhood of, but outside, the beam dump is

$$
\mathcal{R}=7.6 \times 10^{8} \text { stars }^{-1} .
$$

For the long-lived nuclides of concern the activity is given by

$$
A_{i}=\mathcal{R} S_{i}\left(1-\mathrm{e}^{-\lambda_{i} t}\right) \approx \mathcal{R} S_{i} \lambda_{i} t
$$

where $\lambda_{i}=\ln 2 / \tau_{1 / 2}$ is the decay constant of the nuclide and $S_{i}$ is the number per star. The two nuclides of importance are ${ }^{3} \mathrm{H}\left(\tau_{1 / 2}=12.35 \mathrm{yr}\right)$ and ${ }^{22} \mathrm{Na}$ $\left(\tau_{1 / 2}=2.60 \mathrm{yr}\right)$. With estimates of $S\left({ }^{3} \mathrm{H}\right)=0.075$ and $S\left({ }^{22} \mathrm{Na}\right)=0.02$ for interactions in soil [41], the rate of production of the activity is

$$
\begin{aligned}
\frac{d A\left({ }^{3} \mathrm{H}\right)}{d t} & =3.2 \mathrm{MBq} \mathrm{yr}^{-1} \\
\frac{d A\left({ }^{22} \mathrm{Na}\right)}{d t} & =4.0 \mathrm{MBq} \mathrm{yr}^{-1}
\end{aligned}
$$

under the assumption of three times the beam current of the conceptual design. 
Isotope concentrations in ground water are based on community wells, leading to a base quantity from a well of approximately 1000 gallons day $^{-1}$, or $1.4 \times 10^{9}$ $\mathrm{ml} \mathrm{yr}-1$. The resultant concentrations of activity due to the operation of the beam dumps, assuming all the activity nearby the beam dumps gets into the water supply, are

$$
\begin{aligned}
{ }^{3} \mathrm{H} & : 2.3 \mathrm{mBq} \mathrm{ml}^{-1} \\
{ }^{22} \mathrm{Na} & : 0.29 \mathrm{mBq} \mathrm{ml}^{-1}
\end{aligned}
$$

where a leaching factor of 0.10 has been applied to the sodium, based on Ref. 42. Translating to picocuries per milliliter, Table 3.10-2 compares the calculated activities with the EPA standards [43].

Table 3.10-2

Comparison of Calculated and Allowable Maximum Activities

\begin{tabular}{ccc}
\hline \hline & Calculated & 40CFR141 \\
\hline${ }^{3} \mathrm{H}$ & 0.062 & 90 \\
${ }^{22} \mathrm{Na}$ & 0.008 & 0.5 \\
\hline
\end{tabular}

It is clear that, even under extreme assumptions, the beam abort dumps as described in the Conceptual Design protect the ground water system from any radioactive contamination. In addition, of course, the soil and ground water in the vicinity of the dumps will be continuously monitored by the Laboratory Environmental Safety Group. 


\section{ANALYSIS}

The SSC collider consists of a 53-mile long underground tunnel containing two rings of magnets, six interaction regions and two utility regions for the beam injection and abort systems. The facility can also be described for the purpose of this report in terms of arcs, clusters (including interaction regions), and abort regions (see Fig. 1). As previously described, the radiation considerations in each segment are handled separately, but the results of calculations can be summarized in a convenient manner.

According to the loss processes as hypothesized, an envelope of radiation may be generated. The results are graphed according to contours of equal radiation dosage (isodose lines). The meaning of the $10 \mathrm{mrem}$ line, for example, is that this would be the dose equivalent experienced by an individual at that location if the assumed loss scenario actually occurred. Following along the 10 mrem isodose curve to the horizontal axis, one finds the shielding thickness required to reduce the potential dose equivalent to no more than that limit. For the current analysis this is referred to as the "vector."

For the case of radiation generated by hadrons created by scraping the beam at locations in the arcs (and also the other source points) the "width" represents the perpendicular distance away from the loss point where the combined effects of the material in the tunnel wall and the surrounding earth attenuate the dosage to accepted levels. This defines the thickness of the primary shield, shown in Fig. 28 as a circle with 70-foot diameter, as deduced in Section 3.3. The results of similar calculations for the beam dumps and in the interaction regions are given in other sections of Chapter 3. As is shown later, the values used for the boundaries are well outside the calculated widths and vectors for hadron-induced radiation effects.

The determinations are somewhat more complicated for the more weakly interacting muons created by scraping or aborting the beam or at the interaction regions. Using the appropriate isodose curve for muons, one determines the appropriate vector. Moving back along the curve one finds the maximum width derived from the isodose diagram. Thus the elongated diagram produces vectors and widths that can be applied to the different loss points.

As an example, consider the beam dump isodose curves for muons of Fig. 13. For the dump scenario of $2 \times 10^{17}$ protons $\mathrm{yr}^{-1}$, the relevant isodose contour is $5 \times 10^{-20}$. The contour, carried back to $z=0$, has a radius value of about $9 \mathrm{~m} \approx$ 30 feet. From Fig. 14, the vector length is $5.2 \mathrm{~km}$. Thus the muon vector and width for the beam dump are $5.2 \mathrm{~km}$ and $9 \mathrm{~m}$. 


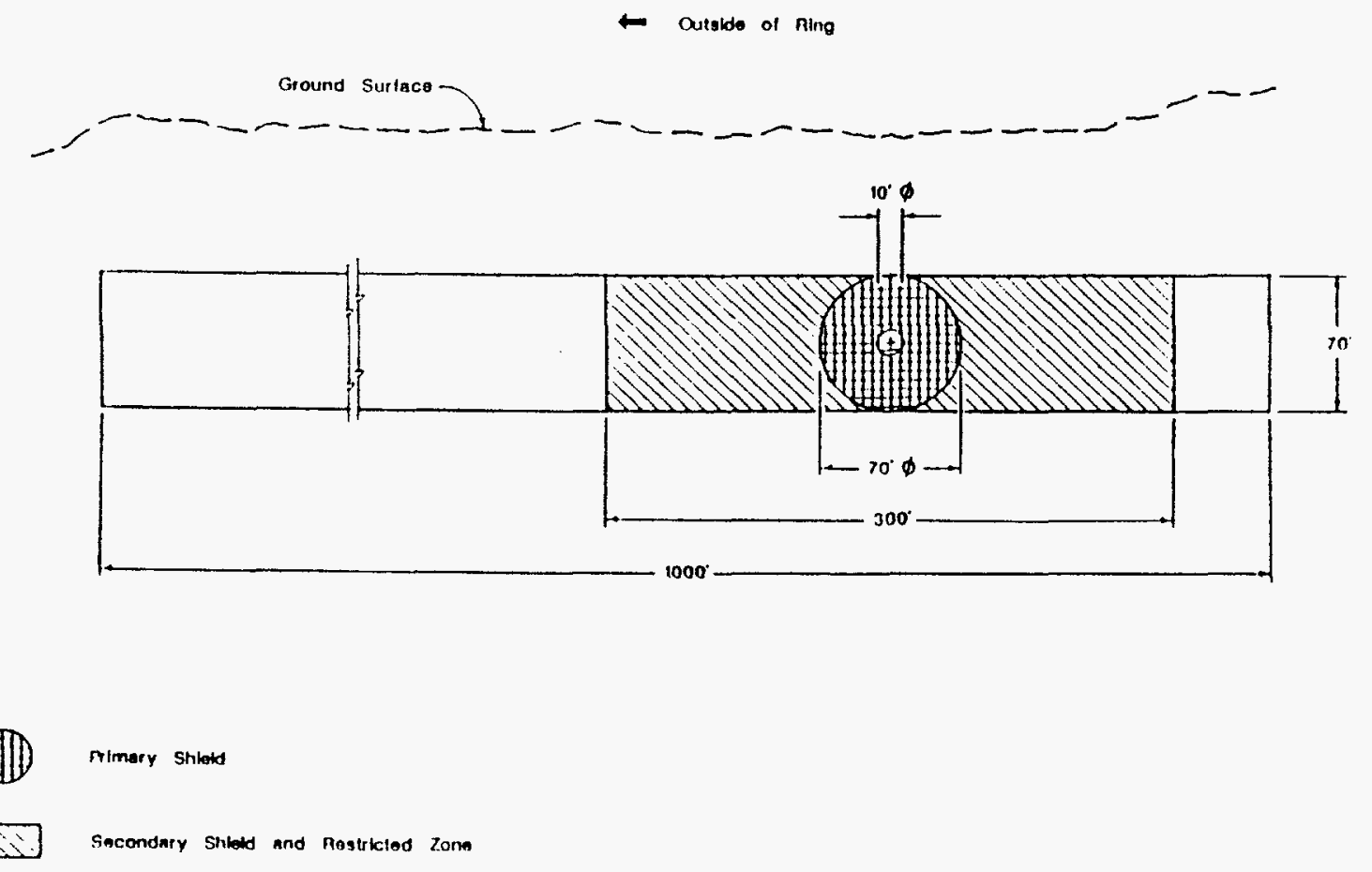

Figure 28. Cross section of region near the tunnel, showing the primary shield of diameter $70 \mathrm{ft}$ around the tunnel and the secondary shield and restricted zone with a total width of $300 \mathrm{ft}$. These lie in the total region $70 \mathrm{ft}$ high and $1000 \mathrm{ft}$ wide that is required for the SSC in the arc regions. 
A detailed layout of the arcs, clusters, abort and major beam cleanup regions was used to identify potential loss points. At all locations the vectors and widths were used to determine the distances of potential radiation influence from the SSC. For the arc regions of the tunnel, a cross section is shown in Fig. 28. The primary shield surrounds the tunnel to a distance of $35 \mathrm{ft}$ in all directions from the tunnel centerline. A further secondary shield and restrictive zone extends horizontally inward and outward to a total distance of $\pm 150 \mathrm{ft}[2]$. There is a further region $70 \mathrm{ft}$ high and totalling $700 \mathrm{ft}$ wide that will be under SSC control, but will generally be subject to shared use. The $1000 \mathrm{ft}$ overall width is required to accommodate minor design changes and for flexibility in the final layout of the accelerator, taking into account the geophysical aspects of the actual site. For the interaction regions, beam dumps and major beam cleanup regions, the loss points are well defined. For the secondary scrapers (in the arcs), the locations are not known precisely at present, but the vectors and widths can be accommodated within the $70 \mathrm{ft}$ by $1000 \mathrm{ft}$ region already specified.

Using the process as described above, the outer boundaries were defined. In the immediate vicinity of the interaction regions, major beam cleanup regions and the beam dumps, the land enclosed by the boundaries is to be acquired by the Federal government. In the arcs and in the muon shield portion of the beam cleanup and dump regions, the land can be shared with current users in those places where the ring tunnel is sufficiently below ground. The shape of the boundaries and the intended use, dedicated or possibly shared, is shown in Fig. 29. The total acreage is 15,830 acres with 7,490 dedicated and 8,340 possibly shared [2].

In order to display how the vectors and widths derived from considerations of muon attenuation were used in the boundary determinations, an expanded view of the cluster region adjacent to the injector complex is shown in Fig. 30 . To illustrate the loss mechanisms that have been studied, several representative vectors are shown. An arc beam scraper vector is represented by arrow 1 , a vector from an interaction region by arrow 2 , and a vector from a beam dump by arrow 3 . The major beam cleanup vectors are not shown. They are close to (and inside) the beam dump vectors and are of shorter length $(2.9 \mathrm{~km}$ at $1 \%$ loss, $3.5 \mathrm{~km}$ at $5 \%$ ). They are thus not a determining factor in defining the land areas. The lengths of these vectors compared to the distances available to the boundaries are shown in Table 4-1.

As previously described, the table is divided into hadron shielding and muon shielding for the three cases of arc, cluster or beam dump regions. For convenience, the intensity or luminosity assumptions used in the calculations are also shown. Results are given both for the beam intensities in the SSC conceptual 


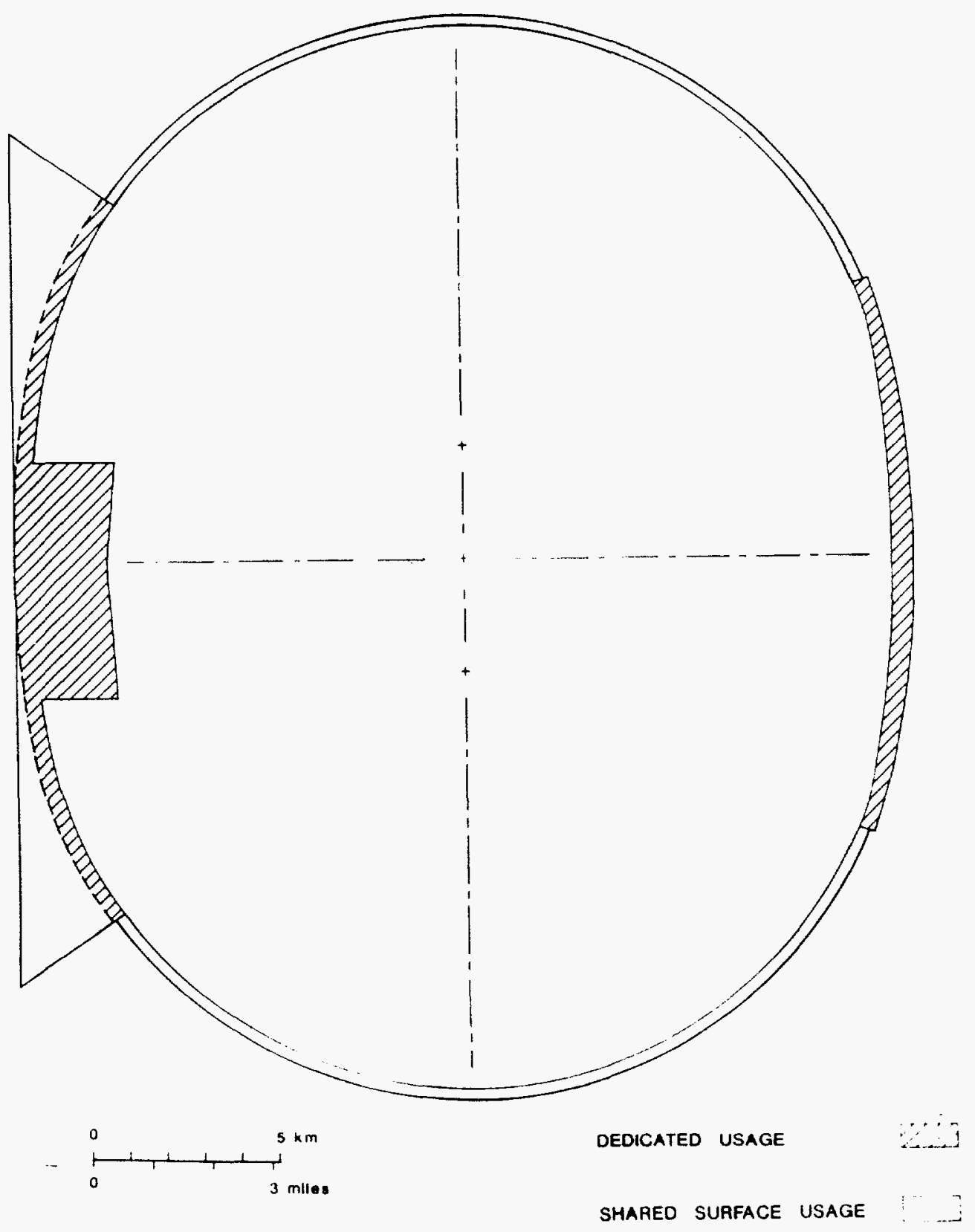

Figure 29. Land required for a collider ring tunnel that is at least $35 \mathrm{ft}$ below the ground surface. Cross-hatched areas are for dedicated use; open areas may have shared use. See Ref. 2 for details. 


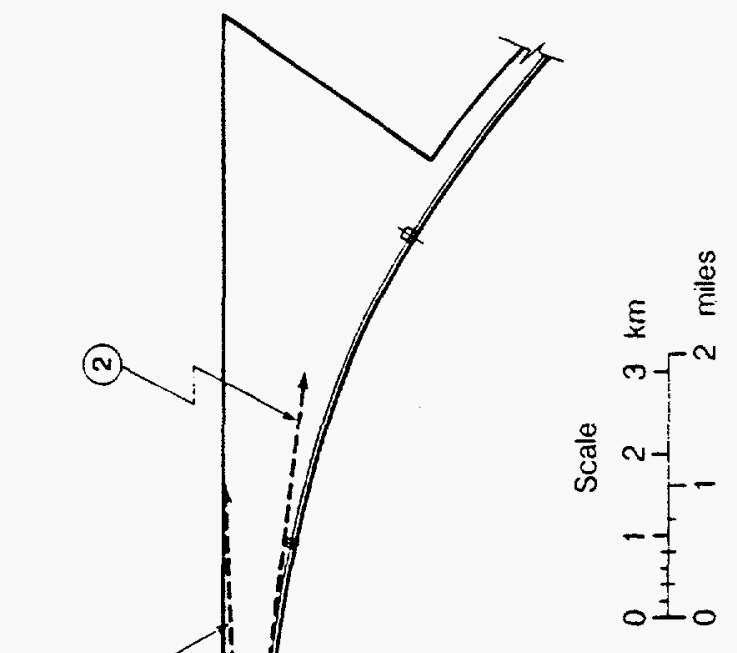

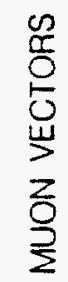

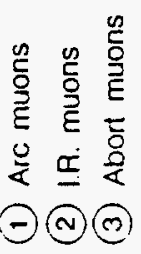

Figure 30. Enlarged view of the lands at the so-called near cluster, with examples of how the shielding vectors delimit the lands for dedicated or shared use. The muon vectors associated with the arcs, interaction regions, and beam dumps are labeled 1,2 , and 3 , respectively. 
Table 4 - 1

Land Distances Derived from Radiation Considerations

Assumptions

\section{Calculated Values dense soil light soil \\ $\left(2.24 \mathrm{gm} / \mathrm{cm}^{3}\right)\left(1.8 \mathrm{gm} / \mathrm{cm}^{3}\right)$ \\ Recommended Dimensions}

HADRON SHIELDING

$\begin{array}{lcccc}\text { Arc (Beam Cleanup) Regions } & 1.3 \times 10^{14} \mathrm{p} / \text { loss } & 21 \mathrm{ft} & 25 \mathrm{ft} & \\ & 3 \times 1.3 \times 10^{14} & 23 \mathrm{ft} & 28 \mathrm{ft} & 30 \mathrm{ft} \\ \text { Interaction Regions } & 10 \times \text { Luminosity } & 26 \mathrm{ft} & 32 \mathrm{ft} & \text { as needed } \\ \text { Beam Dumps } & 3 \times \mathrm{I} & 34 \mathrm{ft} & & 9 \times 9 \times 18 \mathrm{~m}^{3}\end{array}$

MUON SHIELDING

Arc (Beam Cleanup) Regions

Vector

Outer Width

Vector

Width

Cluster Regions

Vector

Outer Width

Vector

Outer Width

Interaction Regions

Transverse Width

Beam Dumps

Vector

Width

Vector

Width
$1.3 \times 10^{14} \mathrm{p} /$ loss $\quad 5410 \mathrm{ft} \quad 6150 \mathrm{ft}$

$1.3 \times 10^{14} \mathrm{p} /$ loss

$380 \mathrm{ft}$

$6530 \mathrm{ft}$

$551 \mathrm{ft}$

$486 \mathrm{ft}$

$7550 \mathrm{ft}$

$736 \mathrm{ft}$

$8120 \mathrm{ft}$

$850 \mathrm{ft}$

$14100 \mathrm{ft}$

$1150 \mathrm{ft}$

$\begin{array}{ccc}10^{33} \mathrm{~cm}^{-2} \mathrm{sec}^{-1} \times 6000 \mathrm{hrs} & 12300 \mathrm{ft} & 15400 \mathrm{ft} \\ 10^{33} \mathrm{~cm}^{-2} \mathrm{sec}^{-1} \times 6000 \mathrm{hrs} & 825 \mathrm{ft} & 1290 \mathrm{ft} \\ 10 \times \text { Luminosity } & 14100 \mathrm{ft} & 17400 \mathrm{ft} \\ 10 \times \text { Luminosity } & 1150 \mathrm{ft} & 1800 \mathrm{ft}\end{array}$

$10 \times$ Luminosity $\quad 36 \mathrm{ft}$

as needed

$15700 \mathrm{ft}$

$19500 \mathrm{ft}$

$500 @ 1.3 \times 10^{14} / \mathrm{yr}$

$25 \mathrm{ft}$

$31 \mathrm{ft}$

$3 \times 1$

$17100 \mathrm{ft}$

$21000 \mathrm{ft}$

$35 \mathrm{ft}$
$18700 \mathrm{ft}$

$>100 \mathrm{ft}$ 
design and for a higher intensity corresponding to ten times the design luminosity. In addition, the vectors are quoted for two typical soil densities. For the arcs, in which the location of beam cleanup areas is not presently specified, the most conservative condition of light soil was used. For the clusters and beam dump regions, where the location of beam loss is well defined, dense soil was assumed. This land would be owned and controlled by the Federal government. If the actual soil has a lower density than assumed, it could be supplemented by denser material. Of course, if the insitu materials were more dense, such as rock or compacted till, this would provide an even more conservative circumstance.

The values in the final column of Table 4-1 are those recommended as siting parameters. They are in some cases $10 \%$ or even more larger than the corresponding numbers from the third or fourth column. This rounding up of the actual 10 mrem calculated values is done to provide additional assurance against the vagaries of extrapolation of the calculations to SSC energies. As discussed in Chapter 2 and Appendix B, there is much experience with the calculational methods and much confidence in their general reliability. Nevertheless, estimates for the SSC are extrapolations beyond existing data. It is prudent to include safety factors to allow for the uncertainties.

The distances from Table 4-1 were used along with detailed collider facility drawings to produce the calculations of acreage found in Ref. 2. It should be noted in summary that the boundaries of the areas were determined on the basis of beam intensity levels well above those specified in the current design of the SSC. Such higher levels of luminosity will only be attained after years of experience with the operation of the facility, if then, but it is sensible to allow for the eventuality from the beginning. Before attempting such increases in beam intensity, extensive measurements of the existing environmental radiation would, of course, be made as a basis for proceeding. 



\section{CONCLUSIONS}

The Task Force on Radiation Shielding has reviewed in detail the assumptions, the calculations and the results on the necessary shielding for operation of the SSC in a safe, environmentally sound manner with respect to radiation exposure. The Task Force has found adequate agreement among predictions of the various tools used to evaluate the hadronic component of the radiation and its degradation in the shielding, as well as agreement between these predictions and experiment. Although there are fewer points of comparison for muons, the agreement between various simulation results and between simulation results and experiment is also satisfactory in this case. In extrapolating to $20 \mathrm{TeV}$, a conservative (high) estimate of the muons, in both number and energy spectrum, was necessary to allow for the greater uncertainties in the muon production, as compared with the hadrons.

The administrative design requirement of not more than 10 mrem per year for the general public has been translated into the transverse and longitudinal dimensions presented in Table 4-1, valid for beam currents 3 times the present design and event rates in the interaction regions 10 times design. As shown in Table 4-1, the dimensions recommended to specify the needed lands for the SSC are equal to, or somewhat larger than, necessary to meet the $10 \mathrm{mrcm} \mathrm{yr}^{-1}$ criterion. This adds a further degree of conservatism to the siting criteria.

The available calculational methods are sufficiently accurate to specify the positions of dose equivalent contours to within $10 \%$ for both hadrons and muons for a given set of input assumptions. Additional work is planned in order to address detailed shielding issues within the site, but the present results are adequate for considerations of the site size and shape and the minimum depth of tunnel.

The Task Force concludes that the environmental shielding requirements of the SSC are well understood and the design incorporates more than adequate shielding in its requirements for land area and configuration. With this design, annual radiation dose equivalent to the general public will not exceed 10 mrem $\mathrm{yr}^{-1}$, an amount small compared to the average exposure from natural sources. The design is such that the SSC laboratory can be operated in a manner that conforms to all governmental regulations regarding radiation exposure to the general public. 


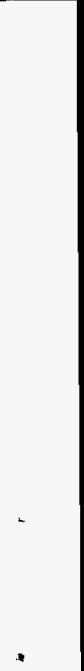




\section{ACKNOWLEDGEMENTS}

The SSC Task Force on Radiation Shielding was requested to reconsider the gross shielding requirements of the SSC and prepare a report in a very short time. The work would have been impossible without the crucial assistance of a number of key individuals. A. Van Ginneken of Fermilab provided many of the calculations that appear in Chapter 3. S. I. Baker of Fermilab contributed to Section 3.10. On short notice F. E. Paige of Brookhaven National Laboratory provided estimates of muon production at $20 \mathrm{TeV}$ using ISAJET. K. Goebel of CERN gave wise counsel and facilitated the participation of G. R. Stevenson on the Task Force and at the Central Design Group during a key period of the work. W. S. Freeman, K. O'Brien, and R. H. Thomas served as consultants to the Task Force.

The Task Force gratefully acknowledges the contributions of these individuals and, by extension, their laboratories, to its work. 



\title{
APPENDIX A
}

\section{A.1. Task Force Membership}

The members of the SSC Task Force on Radiation Shielding are

Murdock G. D. Gilchriese [SSC Central Design Group, on leave from Cornell University]

Donald E. Groom [SSC Central Design Group]

J. David Jackson [University of California, Berkeley (Chairman)]

James R. Sanford [SSC Central Design Group]

Graham R. Stevenson [European Laboratory for Particle Physics (CERN)]

Timothy E. Toohig [SSC Central Design Group]

\section{A.2. Consultants to the Task Force \\ William S. Freeman [Fermi National Accelerator Laboratory] \\ Keran O'Brien [formerly with Environmental Measurements Laboratory, Radiation Physics Division, New York]}

Ralph H. Thomas [Lawrence Berkeley Laboratory]

\begin{abstract}
A.3. Charge by M. Tigner to the Task Force on Radiation Shielding
Using existing matcrials supplemented by further calculation as nceded, provide a report with bibliography that shows how SSC shield dimensions are derived. This report will serve hereafter as a primary reference for SSC radiation shielding and should contain the isodose curves from which the shield dimensions are derived directly, as well as reference to similar calculations and experimental results that lend credence to the SSC conclusions. In addition, some estimate of the uncertainty in the calculations should be made. Both potential doses to persons caused by beam loss or dump events and ground water activation need to be considered.
\end{abstract}





\section{APPENDIX B \\ Comparisons among Calculations and Data}

In the high energy (multi- $\mathrm{TeV}$ ) regime three families of Monte Carlo programs are in general use for shielding calculations: CASIM at Fermilab, FLUKA at CERN and Leipzig, and MARS at Serpukhov. Particularly for following neutrons down to thermal energies the HETC codes from Oak Ridge are also useful. For bulk shielding the empirical formula developed from the work of Moyer is very useful, and extrapolation conditions to high energy have been justified by Thomas and Stevenson [8] and by Cossairt et al. [44]. Descriptions of CASIM, FLUKA and the Moyer Model are found in [8]. Briefer descriptions of CASIM, FLUKA, and HETC are found in Section 2.1 of this report and in Ref. 4.

As the energy of accelerators has increased these programs have evolved to accommodate the improved understanding of production mechanisms and of the development of nuclear cascades. As newer data became available the parameters of the programs were refined accordingly. Calculations of the energy dependence of the side shielding in FLUKA and CASIM based on these developments agree very exactly up into the multi- $\mathrm{TeV}$ range. Thomas has independently derived this parameter for the Moyer model by extrapolation from available experimental data [45]. The extrapolated value is in excellent agreement with the value derived from the codes.

The problems of muon shielding have become increasingly important for shielding the new generation of accelerators in the multi-hundred $\mathrm{GeV}$ regime built in the 1970's. In response to this, CASIM has been modified to include an improved muon capability; for FLUKA and MARS supplementary programs have been written to handle the muon shielding.

For the existing generation of machines the hadron calculations have been extensively cross-checked with one another and to a lesser extent with experimental data up to $800 \mathrm{GeV}$. For the multi-TeV range only limited cross-checking has been published. The extent of intercomparison is indicated in Table B-1. Figs. 31 and 32 from Ref. 47 illustrate the level of agreement between calculational models. Fig. 33 (from Ref. 8) compares FLUKA calculations with experimental data at $200 \mathrm{GeV}$. Fig. 34 from Ref. 44 illustrates the agreement of CASIM calculations of absorbed dose with measurements up to the highest energies currently accessible. In general, the calculations agree with one another and with the data within a factor of two. Since the quantities of interest are typically exponential functions of the shield thickness, a factor of two represents only a small change in shield thickness.

For muons the comparisons are fewer in number. As can be seen in Table B-2, there are only a few published references to muon shielding calculations and 
Table B-1

Hadron Shielding

Intercomparison of Calculations/Validation with Data

\begin{tabular}{lcllll}
\hline \hline $\begin{array}{l}\text { Proton } \\
\text { Energy }\end{array}$ & Ref. & Calculations & Exp.? & Locale & Comment \\
\hline $20 \mathrm{TeV}$ & 46 & CASIM & no & FNAL & Conf. Rep. \\
& 26 & CASIM & no & FNAL & FNAL Pub. \\
& 47 & CASIM/MARS/Moyer & no & FNAL/IHEP N.I.M. Pub. \\
$10 \mathrm{TeV}$ & 26 & CASIM & no & FNAL & \\
& 8 & FLUKA & no & CERN/LBL & IAEA Tech Rep. \\
& 47 & CASIM/FLUKA/MARS & no & FNAL/IHEP & \\
& 38 & CASIM/FLUKA & no & CERN & Internal Rep. \\
$5 \mathrm{TeV}$ & 26 & CASIM & no & FNAL & \\
$3 \mathrm{TeV}$ & 47 & CASIM/MARS & no & FNAL/IHEP & \\
$1 \mathrm{TeV}$ & 48 & Moyer & no & CERN & Nucl. Eng. \& Des. \\
& 8 & CASIM/FLUKA & no & CERN/LBL & \\
& 38 & CASIM/FLUKA & no & CERN & \\
$0.8 \mathrm{TeV}$ & 49 & CASIM/FLUKA/Moyer & no & DESY & Conf. Proc. \\
& 50 CASIM & yes & FNAL & Conf. Rep. \\
& 44 CASIM & yes & FNAL & N.I.M. Pub. \\
$0.5 \mathrm{TeV}$ & 8 & O'Brien & no & EHSL & \\
$0.4 \mathrm{TeV}$ & 50 & CASIM & yes & FNAL & \\
& 44 & CASIM & yes & FNAL & \\
& 47 & CASIM/FLUKA/MARS yes & FNAL/IHEP & \\
$0.3 \mathrm{TeV}$ & 48 Moyer & no & CERN & \\
& 51 Moyer & no & LBL & N.I.M. Pub. \\
& 8 & CASIM/FLUKA & no & CERN/LBL & \\
& 47 & CASIM/MARS & yes & FNAL/IHEP & \\
$0.2 \mathrm{TeV}$ & 51 & Moyer & no & LBL & \\
$0.1 \mathrm{TeV}$ & 8 & CASIM/FLUKA & no & CERN/LBL & \\
& & & & & \\
\hline \hline
\end{tabular}

experiments, even in the regime of currently operating accelerators. One portion of the programs that has been validated is the use of Fermi-Eyges theory for muon transport. Fig. 35 compares CASIM and TOMCAT calculations at 240 $\mathrm{GeV}$ showing good agreement. In Fig. 36 TOMCAT is compared at the same energy with experimental data. Where calculations exist in the TeV range, from 
CASIM and MARS, both the calculations and the presentations are so different as to make comparison difficult. In the CASIM calculations the accelerator is simulated as a continuous dipole with a field of 6 tesla. The magnet cross section and field map are those of a version of the SSC cold-iron, D-style magnet. The presentation is in terms of isodose contours around a tangent vector to the ring from a hypothesized point loss of the entire stored beam. In the MARS case the detailed accelerator lattice is simulated in the program, including quadrupoles and drift spaces, following the work of Keefe and Scolnick during the $200 \mathrm{BeV}$ study [54]. Muon isoflux contours transverse to the ring are calculated assuming a continuous line loss of protons around the entire ring. Fig. 37 illustrates typical output data from the latter approach, while the former forms the basis of this report. Both programs use the same muon source term, discussed in Section 2.3. When converted to shield dimensions, the CASIM results appear to be conservative relative to those from the MARS calculations at the highest energies.

Table B-2

Muon Shielding

Intercomparison of Calculations/Validation with Data

\begin{tabular}{lrlrll}
\hline \hline \multirow{2}{*}{$\begin{array}{l}\text { Proton } \\
\text { Energy }\end{array}$} & Ref. & Calculations & Exp.? & Locale & Comment \\
\hline $20 \mathrm{TeV}$ & 46 & CASIM & no & FNAL & \\
& 26 & CASIM & no & FNAL & \\
& 52 & MARS & no & FNAL/IHEP FN-? in prep. \\
$10 \mathrm{TeV}$ & 26 & CASIM & no & FNAL & \\
$3 \mathrm{TeV}$ & $53 \mathrm{MARS}$ & no & IHEP & N.I.M. Pub. \\
& $26 \mathrm{CASIM}$ & no & FNAL & \\
$1 \mathrm{TeV}$ & 53 MARS & no & IHEP & \\
& 26 & CASIM & no & FNAL & \\
$0.8 \mathrm{TeV}$ & 50 & CASIM & yes & FNAL & \\
$0.28 \mathrm{TeV}$ & 30 & TOMCAT & yes & CERN & N.I.M. Pub. \\
$0.24 \mathrm{TeV}$ & 30 & CASIM/TOMCAT yes & CERN/LBL & N.I.M. Pub. \\
\hline \hline
\end{tabular}

The agreement between CASIM muon calculations and experimental data at 400 and $800 \mathrm{GeV}$ is illustrated in Fig. 38 from Ref. 50. In the momentum range where the spectrometer has adequate acceptance, the agreement is quite satisfactory. 


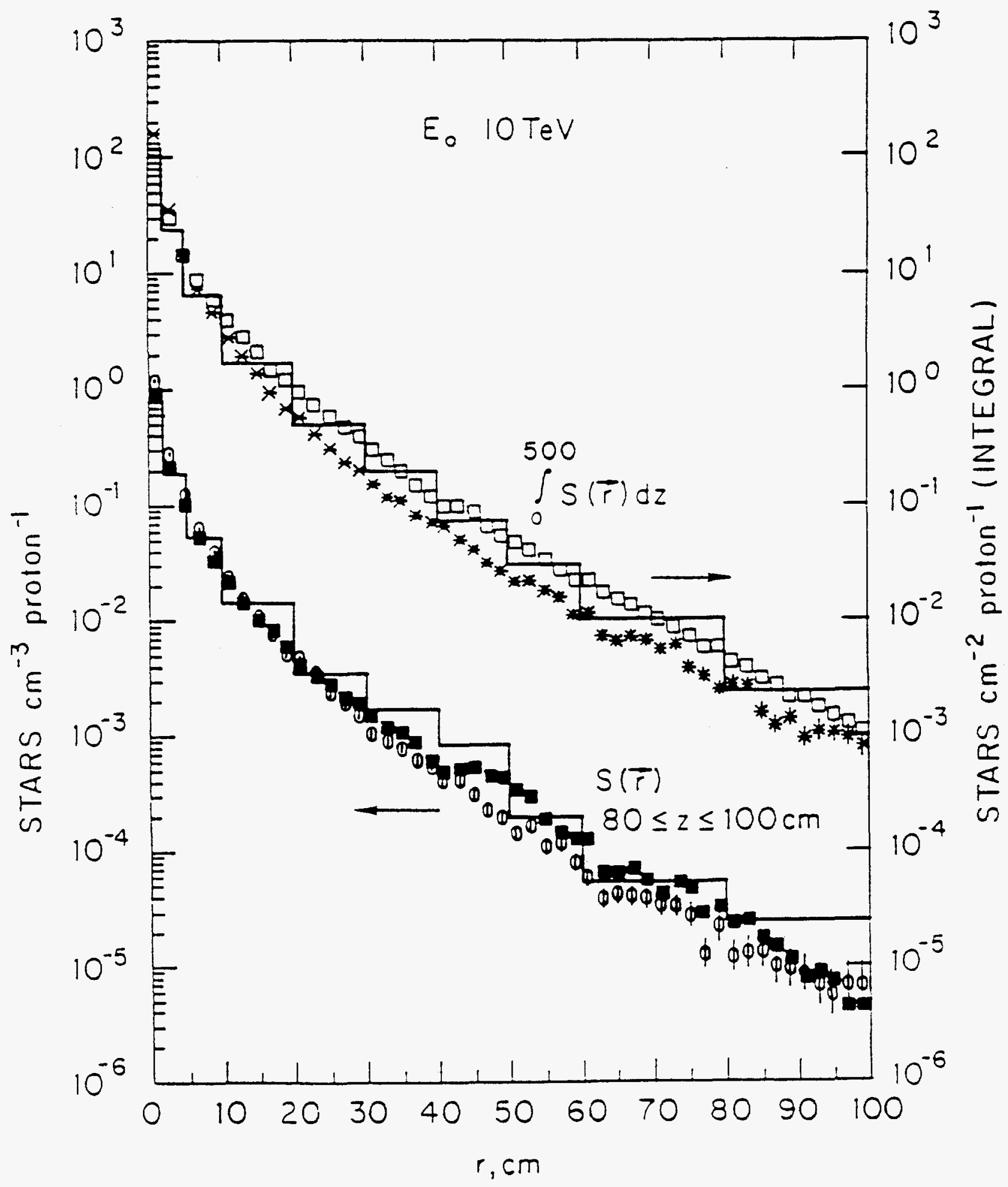

Figure 31. Hadron shiclding, $10 \mathrm{TeV}$, calculated lateral distributions of the star density in an iron beam dump with a length of $500 \mathrm{~cm}$ and a diameter of $200 \mathrm{~cm}$ : stars $\mathrm{cm}^{-2}$ for the longitudinally integrated distribution, stars $\mathrm{cm}^{-3}$ for the values at the maximum of the cascade development. The histograms are MARS calculations, the squares are CASIM results and the asterisks and open circles are FLUKA results. (See Ref. 4T.) 


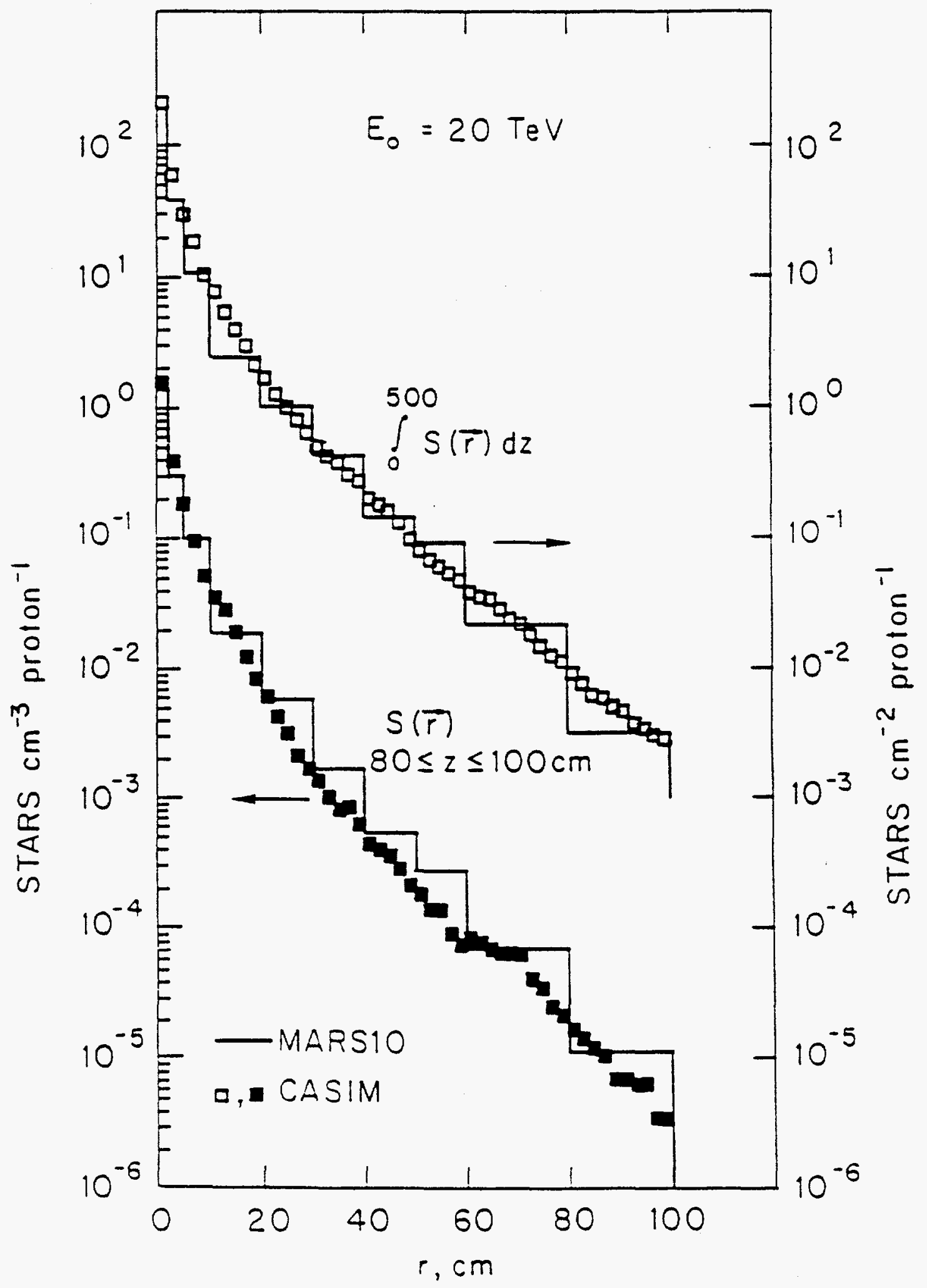

Figure 32. Hadron shielding, $20 \mathrm{TeV}$. Similar to the preceding figure, but only MARS and CASIM are compared, as indicated. (See Ref. 47.) 


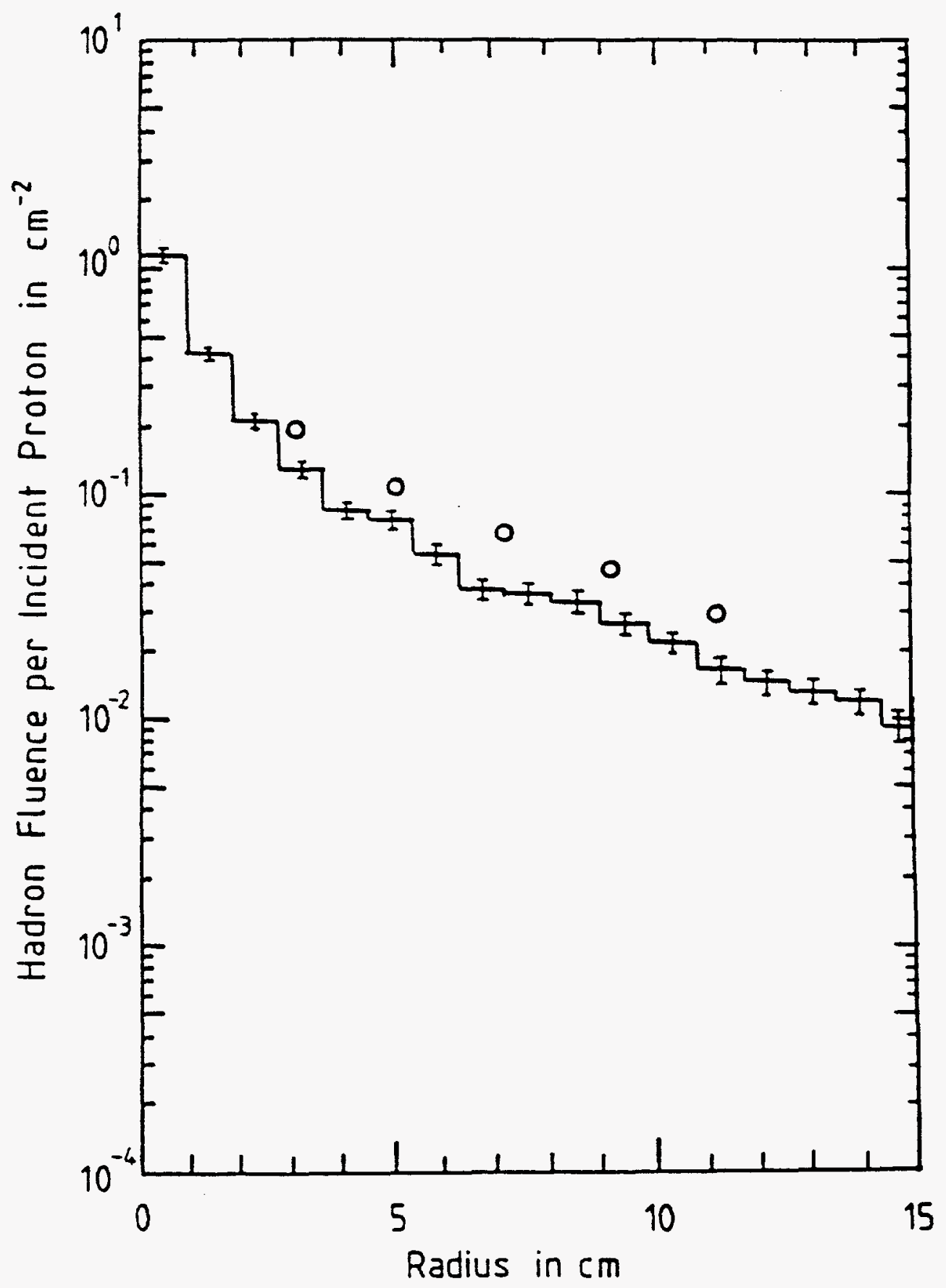

Figure 33. Hadron shielding, $200 \mathrm{GeV}$. Hadron fluence as a function of radius in an aluminum beam stop. The histogram with error bars represents calculations using FLUKA; the open circles are experimental measurcments. (Sce Ref. S.) 


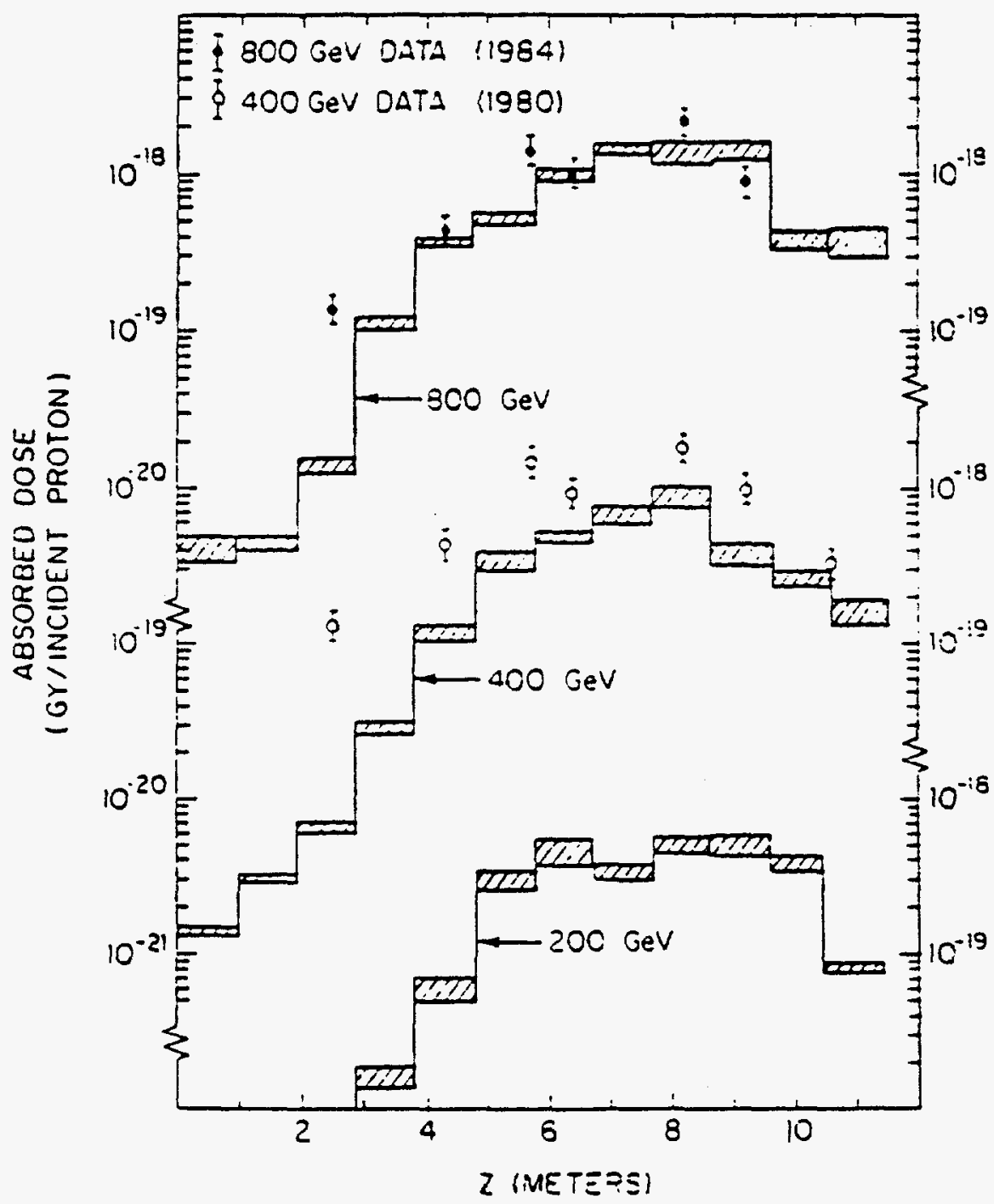

Figure 34. Hadron shielding, 400 and $800 \mathrm{GeV}$. The absorbed radiation dose outside an earth and concrete shield alongside an iron beam stop as a function of the distance along the beam line. The histograms represent calculations using CASIM of the dose for three incident proton momenta; the circles with error bars are the experimental data at 400 and $800 \mathrm{GeV}$. (See Ref. 50.) 


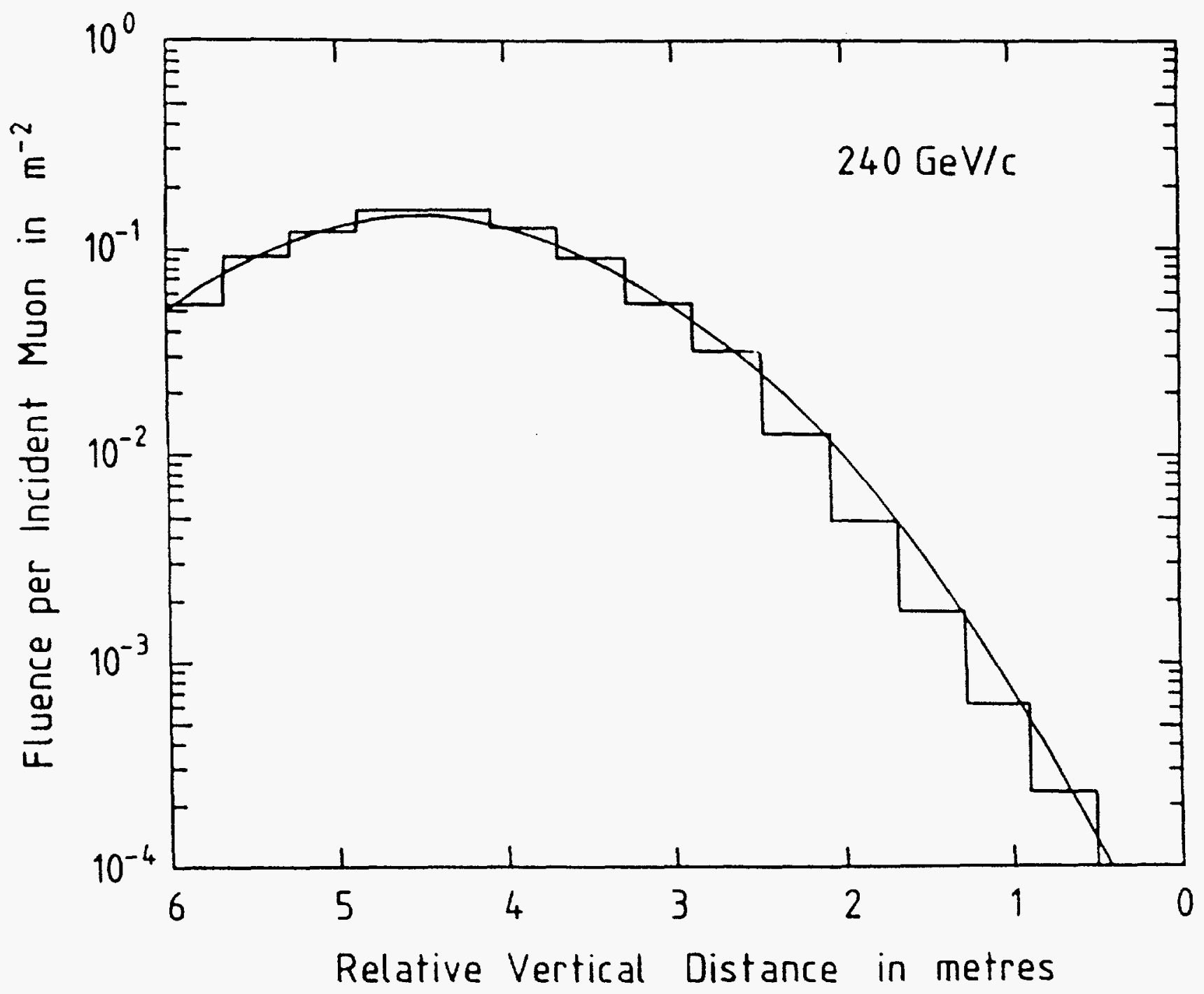

Figure 35. Muon shielding, $240 \mathrm{GeV}$. The variation of muon fluence as a function of relative vertical distance; comparison between TOMCAT (continuous line) and muon transport in CASIM [30]. 


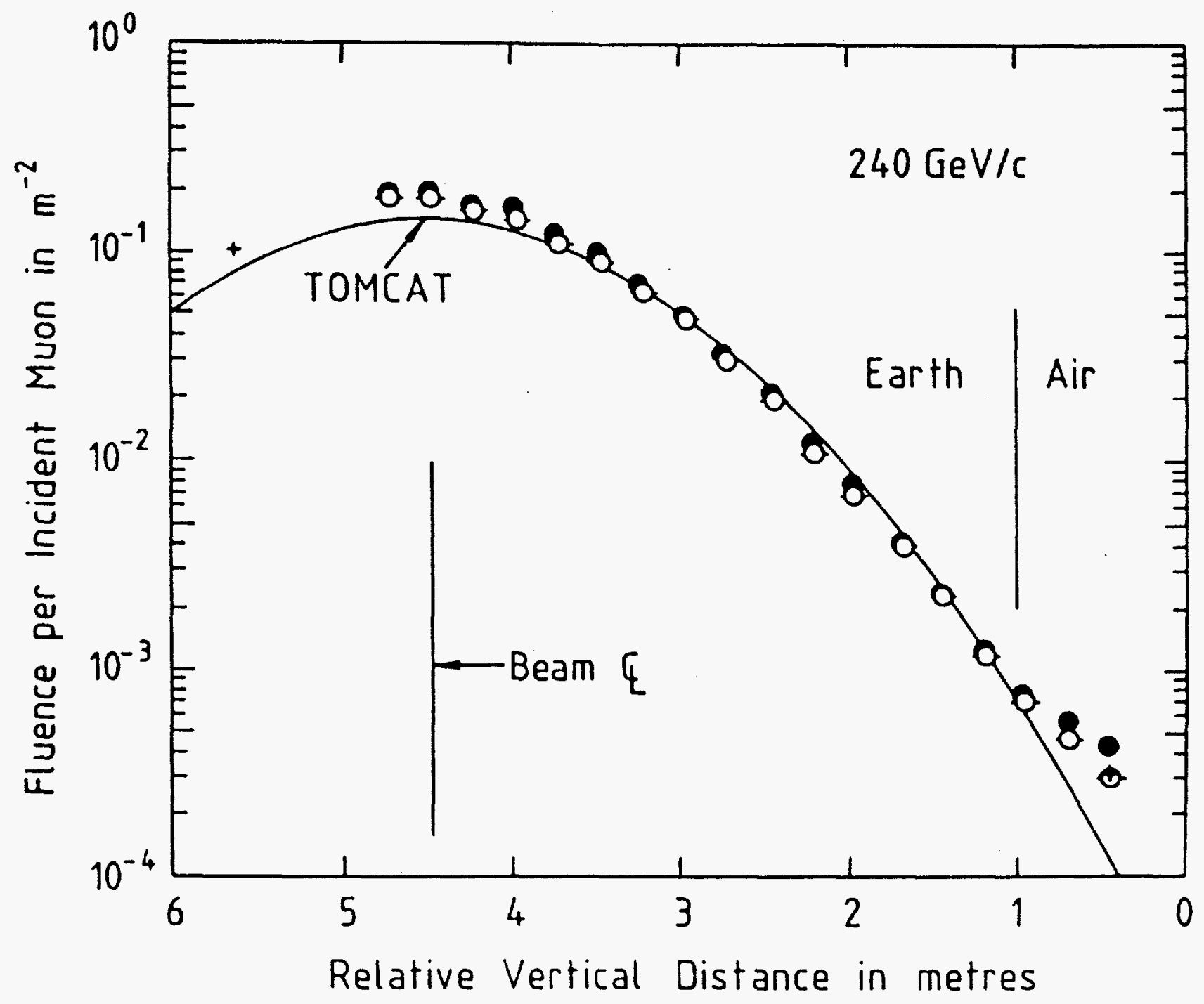

Figure 36. Muon shiclding, $240 \mathrm{GeV}$. The variation of muon fluence as a function of relative vertical distance: comparison between TOMCAT (continuous line) and experimental data (circles). (Sce Ref. 30.) 


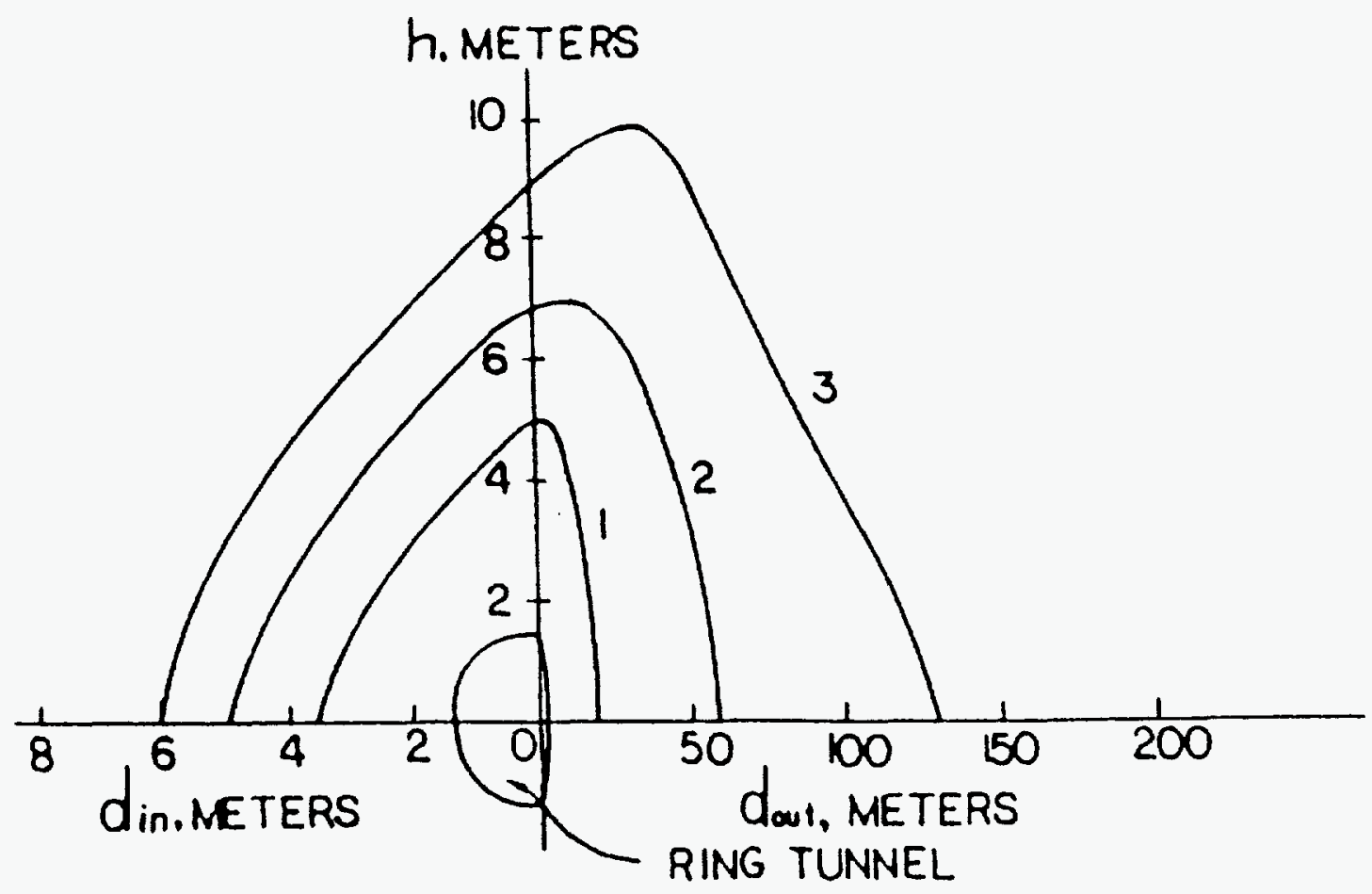

Figure 37. Muon shielding, $1 \mathrm{TeV}$. Calculated muon isofluence contours in soil around the Fermilab Tevatron ring due to uniform $1 \mathrm{TeV}$ proton losses using the MARS program. Curve 1 represents a muon flux of $10^{-8}$ $\mathrm{cm}^{-2}$, for one proton $\mathrm{m}^{-1}$ loss. Curve 2 is the $10^{-9}$ isofiux contour, and curve 3 the $10^{-10}$ contour. (See Ref. 53.) 


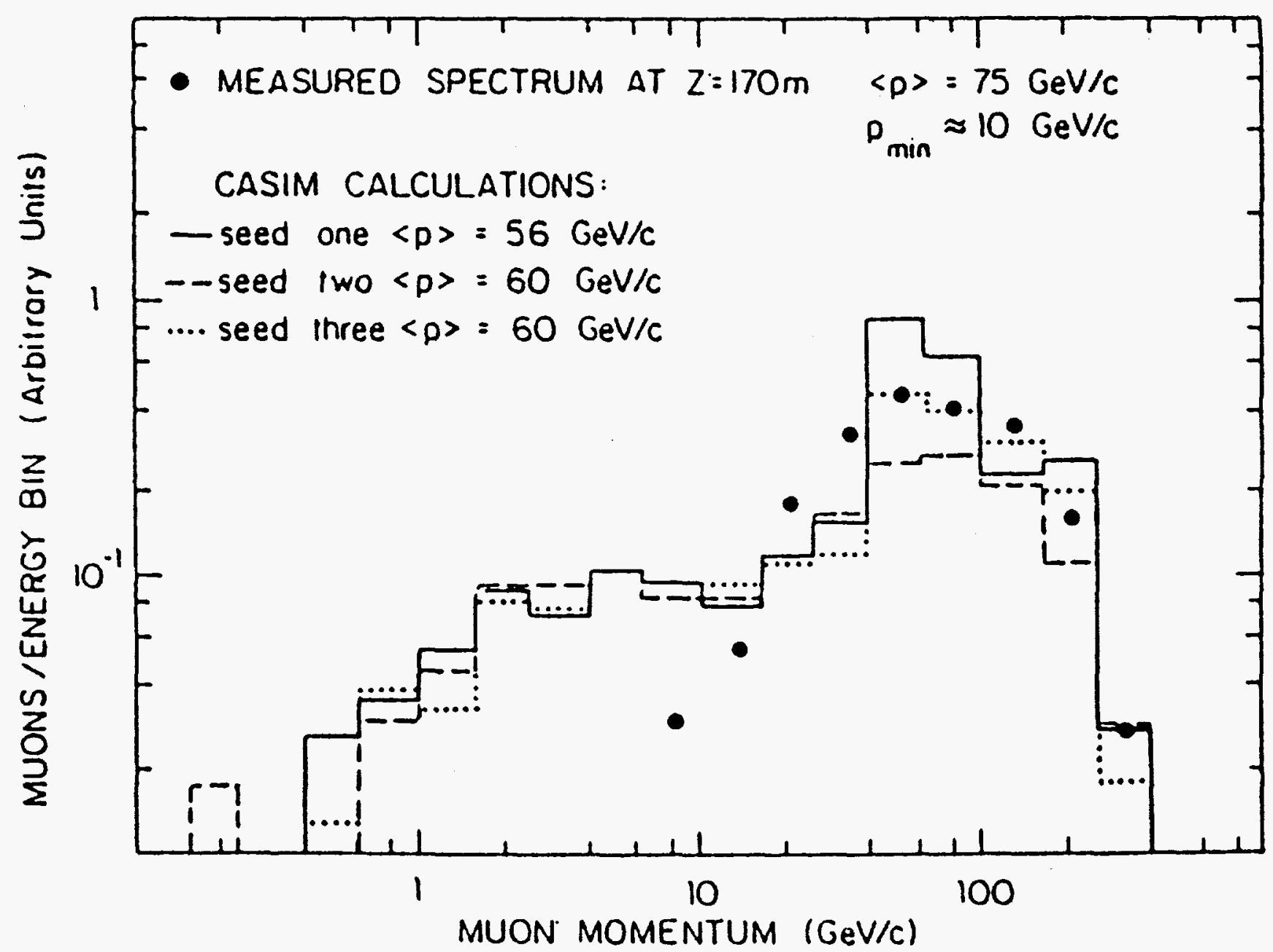

Figure 38. Muon shielding, $800 \mathrm{GeV}$. A comparison of muon momentum spectra calculated using CASIM with measurements made in a large magnetic spectrometer. The spectrometer had a low-momentum cutoff of about $10 \mathrm{GeV} / \mathrm{c}$. The muons were produced by an $800 \mathrm{GeV}$ proton beam striking a dump. Histograms represent different random number initial values (seeds) for the CASIM calculations. (See Ref. 50.) 



\section{APPENDIX C \\ Radioactivity Induced in \\ Nearby Well by Accidental Loss of Beam}

It is anticipated that most of the 1000 - $\mathrm{ft}$ wide swath around the SSC ring will be land with shared use. As shown in Fig. 28, there is a primary shield 70 feet in diameter around the tunnel, a secondary shield or restricted zone extending 150 feet horizontally on either side of the tunnel. Penetrations or activities within the restricted zone are to be prohibited, unless specifically authorized (see Ref. 2, p. 44). To provide a framework for decisions on continued or future proposed use, especially for water wells, we develop here a model of the level of radioactivity induced in a nearby well (as a function of its distance from the tunnel) by the accidental loss of the full beam.

(a) Elementary hydrology of a shallow well

Shallow wells are assumed to draw water from the surrounding soil over a depth down to some base layer of impervious material. While large scale wells often span complex and varied sub-surface conditions, a small well can be modeled in the simple way shown in Fig. 39. The relevant hydrological geometry is considered to be cylindrically symmetric, with the well dug down to the horizontal impervious layer and with the water table somewhere between the surface and the impervious stratum.

The profile of depth of water $h(r)$ (defined by the height of water in an adjacent test well dug a distance $r$ away) is governed by the assumed steady rate of pumping of $Q$ gallons per day through conservation of the flow of water through successive concentric cylinders. The flux is determined by the gradient relation, $S_{r}=k d h(r) / d r$, where $S_{r}$ is the inward flux at radius $r$ and $k$ is a constant with dimensions of volume per unit time per unit area ( $k$ is characteristic of the soil and is variously expressed as gallons per day per square foot or feet per day). Conservation of water yields the steady-state equation,

$$
Q=2 \pi r h(r) S_{r}=2 \pi k r h \frac{d h}{d r}=\pi k \frac{d\left(h^{2}\right)}{d(\ln r)}
$$

The solution is for $h(r)$ as a function of $r$ is

$$
Q \ln \left(r / r_{0}\right)=\pi k\left[h^{2}(r)-h_{0}^{2}\right]
$$

where $r_{0}$ is the radius of the well and $h_{0}$ is the height of the water above the impervious layer at the well (see Fig. 39). 


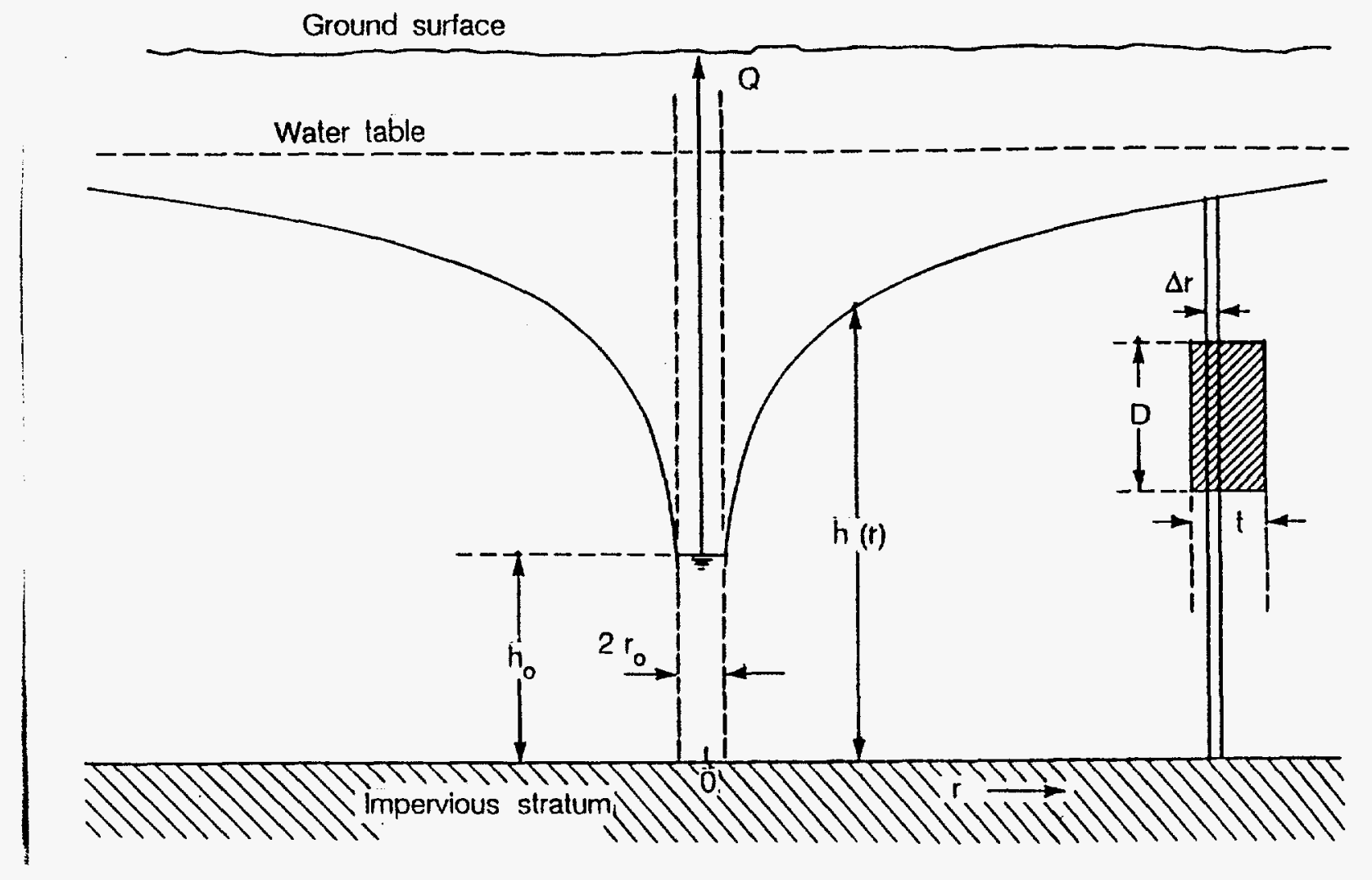

Figure 39. Hydrological model of a shallow well in proximity to the tunnel where an unintended loss of beam occurs. The radioactive region is represented in cross section by the shaded rectangle on the right. 
If $H$ is the depth of the impervious layer below the water table, the radius of influence $R$ of the well can be defined by the standard relation $[55,56]$,

$$
Q=\frac{\pi k\left(H^{2}-h_{0}^{2}\right)}{\ln \left(R / r_{0}\right)}
$$

For our purposes the detailed solution is not needed, only the conservation of water.

(b) Total activity generated by beam loss

First we model the primary radioactivity that eventually may appear in the water supply. It is postulated that there is an accidental loss of the full beam (3 times design current) at full energy at one region somewhere along the arcs. The hadronic interactions caused by the $4 \times 10^{14}$ protons produce nuclear stars (leading to some radioactive species) in the tunnel walls and in the soil immediately surrounding the tunnel. The longitudinal extent of appreciable star production is some 10 meters. Fig. 23 shows the longitudinally integrated star density, times $2 \pi \rho$, as a function of radial distance into the soil. The quantity plotted is the number of stars per incident proton per $\mathrm{cm}$,

$$
\frac{d N_{s}(\rho)}{d \rho}=2 \pi \rho \int n_{s}(z, \rho) d z
$$

where $n_{s}(z, \rho)$ is the nuclear star density. The $20 \mathrm{TeV}$ curve in Fig. 23 can be fitted (for the case of light soil, with density $1.8 \mathrm{~g} \mathrm{~cm}^{-3}$ ) by

$$
\frac{d N_{s}}{d \rho}=160 \times\left[0.687 e^{-\left(\rho-\rho_{t}\right) / 30}+0.313 e^{-\left(\rho-\rho_{t}\right) / 60}\right]
$$

where $\rho_{t}$ is the tunnel radius and distances are in $\mathrm{cm}$. The total number of stars per proton is found to be

$$
d N_{s}=6.3 \times 10^{3} \mathrm{stars} / \text { proton },
$$

and the mean thickness of deposition to be $40 \mathrm{~cm}$. With $4 \times 10^{14}$ protons, the total number of stars produced is

$$
N_{s}=2.5 \times 10^{18} \text { stars }
$$

A significant fraction of these stars will be in the concrete tunnel wall and will not reach the ground water, but to err on the conservative side we assume all of the stars are in the permeable soil outside the actual tunnel. 
The radioactive nuclides of concern for the groundwater are ${ }^{3} \mathrm{H}$ and ${ }^{22} \mathrm{Na}$. These are produced in abundances of 0.075 and 0.02 per star, respectively (see Table 3.10-1). Most of the tritium is available to migrate into the water, but less than $10 \%$ of the sodium-22 can be leached from the soil [42]. With the decay constants from Table $3.10-1$ and leaching factors of $100 \%$ and $10 \%$, respectively, we find the following total initial ${ }^{3} \mathrm{H}$ and ${ }^{22} \mathrm{Na}$ activities potentially available for contamination of water from a loss of beam,

$$
\begin{aligned}
A\left({ }^{3} \mathrm{H}\right) & =3.4 \times 10^{8} \mathrm{~Bq}\left(=9.1 \times 10^{9} \mathrm{pCi}\right) \\
A\left({ }^{22} \mathrm{Na}\right) & =4.3 \times 10^{7} \mathrm{~Bq}\left(=1.2 \times 10^{9} \mathrm{pCi}\right)
\end{aligned}
$$

The region of deposition of this activity is perhaps 10 or 20 meters in length and less than one meter in radius outside the tunnel. It is likely to be deposited somewhat asymmetrically on one side or the other. With a tunnel diameter of $3 \mathrm{~m}$, we model the deposition volume as being rectangular, $4 \mathrm{~m}$ high and $3 \mathrm{~m}$ wide in cross section and of length $20 \mathrm{~m}$ (although the length does not enter the calculations) and assume the activity to be uniformly distributed throughout the volume.

(c) Specific activity in the well

We suppose that there is a nearby well, a radial distance $r$ away from the region of deposition of the radioactivity. We assume, contrary to expectations, * that the tunnel lies below the water table and that the deposition region lies within the radius of influence of the well, right in the aquifer being drawn on, as shown in Fig. 39. The more likely situation of the tunnel above the water table will lead to even lower specific activities in well water than found in the present calculation.

The amount of activity drawn into the well is determined by the rate of pumping $Q$ and the necessary flow through a cylinder of radius $r$ and height $h(r)$. Let $\Delta V$ be the volume of soil yielding $Q$ gallons of water. The cylindrical shell providing this amount of water will be of radial thickness $\Delta r$, where $\Delta V=$ $2 \pi r h(r) \Delta r$. The fraction $F$ of the volume of activity included in this shell can be seen from Fig. 39 to be

$$
F=\frac{\Delta r}{t}=\frac{\Delta v}{2 \pi r t h}
$$

provided $\Delta r<t$.

* It is expected that over most of the circumference the tunnel will lie well above the water table or will go through relatively impervious rock where it is not. There may be, however, local regions where the water table is above the tunnel and a well and an accidental loss of beam might coincide. 
The specific activity ( $\mathrm{Bq}$ per unit volume) of nuclide $i$ in the well water is therefore

$$
a_{i}=F \frac{A_{i}}{Q}=\frac{F A_{i}}{p \Delta V}=\frac{f}{p}\left(\frac{1}{2 \pi r t D}\right) A_{i}
$$

where $f=D / h$ is the fraction of the total height of the cylindrical shell occupied by the activated region and $p$ is the effective porosity of the soil. Note that to obtain $a_{i}$ in $\mathrm{pCi} \mathrm{ml}{ }^{-1}$, we must put lengths in centimeters and $A_{i}$ in picocuries. The result for $a_{i}$ does not depend explicitly on the pumping rate $Q$, but does implicitly through $f$.

Porosity values vary widely for rocks and soils, but are typically in the range $p=0.20-0.35$ (Ref. 56, Chapter 2). We take a value of $p=0.2$ for our calculations.

The fractional height factor $f$ cannot be larger than one and in any reasonable circumstance will be considerably smaller. In the absence of specific site information we use the height of the restricted zone as an estimate of $h(r)$. Then $f \simeq 4 / 21 \simeq 0.2$. We choose $f / p=1.0, D=400 \mathrm{~cm}$, and $t=300 \mathrm{~cm}$. This gives

$$
\frac{f}{p}(2 \pi r t D)^{-1}=\frac{1.3 \times 10^{-8}}{r(\mathrm{~m})} \mathrm{cm}^{-3} \text {. }
$$

This yields specific activities for ${ }^{3} \mathrm{H}$ and ${ }^{22} \mathrm{Na}$ as a function of distance $\mathrm{r}$ for a loss of 3 times the design beam current,

$$
\begin{aligned}
a\left({ }^{3} \mathrm{H}\right) & =\frac{121}{r(\mathrm{~m})} \mathrm{pCi} \mathrm{ml}^{-1} \\
a\left({ }^{22} \mathrm{Na}\right) & =\frac{15}{r(\mathrm{~m})} \mathrm{pCi} \mathrm{ml}^{-1}
\end{aligned}
$$

The EPA standards [43] are $90 \mathrm{pCi} \mathrm{ml}^{-1}$ and $0.5 \mathrm{pCi} \mathrm{m}^{-1}$, respectively.

Fig. 24 in the text shows the two specific activities, relative to the EPA values, as a function of distance from the tunnel. The tritium activity is below the EPA value for all distances that make sense $(r>1.3 \mathrm{~m})$. The sodium-22 activity is below the EPA standard for $r>10 \mathrm{~m}$ at the design beam current and $r>30 \mathrm{~m}$ for 3 times the design current. Since the ${ }^{22} \mathrm{Na}$ level is the governing factor, we can define

$$
r_{\mathrm{EPA}}=10 \frac{f}{p} \frac{I}{I_{\mathrm{D}}} \text { meters }
$$

as the standards of distance, where $I$ is the actual beam current at the time of loss and $I_{\mathrm{D}}$ is the design current. This distance depends on the parameters in the model, but is representative in the absence of specific site information. 


\section{REFERENCES}

1. Conceptual Design of the Superconducting Super Collider, SSC Central Design Group Report SSC-SR-2020 (1986).

2. U. S. Department of Energy Report No. DOE/ER-0315, Invitation for Site Proposals for the Superconducting Super Collider, April 1987.

3. J. D. Cossairt, Shielding Calculations for the B-0 Colliding Detector Area, Fermilab Report No. TM1016, 7 June 1982; J. D. Cossairt, Film Badge Requirements for the B-0 Assembly Hall, Fermilab, Memorandum to R. F. Schwitters et al, 26 December 1984.

4. SSC Workshop on Environmental Radiation, 14-18 October 1985, SSC Central Design Group Report SSC-SR-1016 (January 1986).

5. A. Van Ginneken and M. Awschalom, High-energy Particle Interactions in Large Targets: Volume 1; Hadronic Cascades, Shielding, Energy Deposition, Fermilab Report (unnumbered) (1974).

6. B. J. Moyer, Evaluation of Shielding Required for the Improved Bevatron, Lawrence Radiation Laboratory Report UCRL-9769 (June 1961); B. J. Moyer, in Premier Colloque International sur la Protection Auprès des Grands Accèlèrateurs, p.61 (Presses Universitaires de France, Paris, 1962).

7. S. J. Lindenbaum, Ann. Rev. Nucl. Sci. 11, 213 (1961).

8. R. H. Thomas and G. R. Stevenson, Radiological Safety Aspects of the Operation of Proton Accelerators, IAEA Technical Report Series (1987) (to be published).

9. A. Van Ginneken, Fermilab Report FN-272, Fermilab, Batavia, IL, USA (1975).

10. R. Hagedorn, Suppl. Nuovo Cim. 3, 147 (1965); R. Hagedorn and J. Ranft, Suppl. Nuovo Cim. 6, 169 (1968); J. Ranft, Secondary Particle Spectra According to the Thermodynamical Model. A Fit to Data Measured in p-nucleus Collisions, TUL-36, Karl Marx Univ., Leipzig, DDR (1970).

11. A. Van Ginneken, Fermilab Report FN-309 (1978).

12. A. Van Ginneken, Nucl. Instr. Meth. A251, 21-39 (1986); see also S. Qian and A. Van Ginneken, Characteristics of Inelastic Interactions of High Energy Hadrons with Atomic Electrons, Fermilab Report Fermilab-Pub$86 / 145$ (1986), to be published in Nucl. Instr. and Meth.

13. N. V. Mokhov, IHEP Preprint 82-168, Serpukov, USSR (1982); A. N. Kalinovsky, N. V. Mokhov and Yu. P. Nikitin, Penetration of High Energy Particles through Matter, (Energoatomizdat, Moscow, USSR) (1984). 
14. P. A. Aarnio, J. Ranft and G. R. Stevenson, CERN Internal Report TISRP/106-Rev (1984).

15. P. A. Aarnio, A. Fasso, H-J. Moehring, J. Ranft and G. R. Stevenson, CERN TIS-RP/168 (1986).

16. J. Ranft, J. J. Möhring, T. M. Jenkins and W. R. Nelson, The Hadron Cascade Code, FLUKA82: Setup and Coupling with EGS4 at SLAC, Stanford Linear Accelerator Center Report SLAC-TN 86-3 (April 1986).

17. H. -U. Bengtsson and T. Sjöstrand, in preparation (description of Pythia version 4.6); H. -U. Bengtsson, in Proc. of the UCLA Workshop on Observable Standard Model Physics at the SSC: Monte Carlo Simulation and Detector Capabilities, H-U Bengtsson, C. Buchanan, T. Gottschalk, and A. Soni, eds., World Scientific Publishing Col, Singapore (1986).

18. F. E. Paige and S. D. Protopopescu, in Proc. of the UCLA Workshop on Observable Standard Model Physics at the SSC: Monte Carlo Simulation and Detector Capabilities, H-U Bengtsson, C. Buchanan, T. Gottschalk, and A. Soni, eds., World Scientific Publishing Col, Singapore (1986).

19. M. B. Emmett, Oak Ridge National Laboratory Report ORNL-4972 (1975).

20. K. C. Chandler and T. W. Armstrong, Oak Ridge National Laboratory Report ORNL4744 (1972).

21. T. Sjöstrand, Fermilab preprint, Fermilab-Pub-85/119-T (1985); to be published in Proc. 23rd Int. Conf. on High Energy Phys., Berkeley CA, S. Loken, ed. (World Scientific) (to be published.)

22. M. M. Block and R. N. Cahn, Rev. Mod. Phys. 57, 563 (1985).

23. K. Goulianos, Phys. Rep. 101, 169 (1985).

24. D. Carlsmith, D. Groom, D. Hedin, T. Kirk, T. Ohsugi, D. Reeder, J. Rosner, and S. Wojcicki, Proc. of the 1986 Summer Study on the Physics of the Superconducting Supercollider, R. Donaldson and J. Marx, eds. Div. of Part. and Fields, Am. Phys. Soc. (to be published, 1987).

25. A. Van Ginneken, private communication (September 1984).

26. A. Van Ginneken, P. Yurista and C. Yamaguchi, Fermilab Note FN-447; also published as SSC Central Design Group Report SSC-106 (January, 1987).

27. J. L. Ritchie et al., Phys. Rev. Lett. 44, 230 (1980) (See footnote 9).

28. F. E. Paige, private communication (February 1987).

29. G. R. Stevenson, CERN Report CERN LABII-RA/Note/74-14 (1974). 
30. W. R. Nelson, T. M. Jenkins, G. R. Stevenson, M. Nielson, E. H. M. Heijne, P. Jarron, J. Lord, and S. Anderson, Nucl. Instr. Meth. 215, 385-396 (1983).

31. G. R. Stevenson, Health Phys. 47, 837 (1984).

32. T. A. Gabriel and R. T. Santoro, Nucl. Instr. Meth. 95, 275 (1971).

33. International Commission on Radiological Protection, Data for Protection Against Ionizing Radiation from External Sources: Supplement to ICRP Publication 15, ICRP Publication 21, Pergamon Press (Oxford and New York) (1973).

34. G. R. Stevenson, Dose Equvalent per Star in Hadron Cascade Calculations, CERN Report TIS-RP/173 (1986).

35. K. O'Brien and J. E. McLaughlin, Nucl. Instr. Meth. 60, 129 (1968); K. O'Brien, Neutron Spectra in the Side-shielding of a Large Particle Accelerator, Health and Safety Laboratory Report, New York, HASL-240 (1971).

36. International Commission on Radiation Units and Measurements, Determination of Dose Equivalents Resulting from External Radiation Sources, ICRU Report 39, ICRU Publications (Bethesda, MD) (1985).

37. G. R. Stevenson, private communication (February 1987).

38. G. R. Stevenson, Empirical Parametrization of the Shielding of End-Stops at High-Energy Proton Accelerators, CERN Internal Report TIS-RP / 183/ CF (January 1987).

39. International Commission on Radiation Protection, Limits on Intakes of Radionuclides by Workers, Pub. 30 (Pergamon Press, Oxford) (supplements issured regularly).

40. L. Jones, Radiation Safety of the Superconducting Supercollider, SSC Central Design Group Report SSC-54 (April 1986).

41. P. J. Gollon, Soil Activation Calculations for the Anti-Proton Target Area, Fermilab Report TM-816 (September 1978).

42. T. Borak et al., Health Phys. 23, 679-687 (1972); S. Baker, in Proc. Srd Env. Prot. Conf., Chicago, Sept. 23-26, 1975, CONF-750967, ERDA 92, p. 329-347; S. Baker, private communication (1987).

43. Environmental Protection Agency 40 CFR Part 141: EPA Advance Notice of Proposed Rulemaking Dealing with Radionuclides Under Safe Drinking Water Act (51 FR 34836; Sept. 30, 1986).

44. J. D. Cossairt, S. W. Butala, and M. A. Gerardi, Nucl. Instr. Meth. A238, 504-508 (1985). 
45. R. H. Thomas and S. V. Thomas, Health Phys. 46, 954 (1984).

46. J. D. Cossairt, and A. J. Elwyn, Shielding Considerations for Fixed Target Usage of the SSC, Proc. of the Workshop on Fixed Target Physics, Woodlands, TX, p. 19 (1984).

47. N. V. Mokhov, and J. D. Cossairt, Nucl. Instr. Meth. A244, 349 (1986).

48. J. T. Routti, and M. H. Van de Voorde, Nucl. Eng. and Design 21, 421-434 (1972).

49. H. Dinter, and K. Tesch, Radiation Protection for the HERA Colliding Beam Facility, Proc. Health Physics of Radiation Generating Machines, CONF-8602106 pp. 597-604 (1987).

50. J. D. Cossairt, Shielding Design at Fermilab; Calculations and Measurements, Proc. Health Physics of Radiation Generating Machines, CONF8602106 pp. $942-658$ (1987).

51. J. T. Routti, and R. H. Thomas, Nucl. Instr. Meth. 76, 157-163 (1969).

52. N. V. Mokhov, and T. E. Toohig, Muons; Siting the SSC, Fermilab FN (in preparation).

53. M. A. Maslov, N. V. Mokhov, and A. V. Uzunian, Nucl. Instr. Meth. 217, 419-424 (1983).

54. D. Keefe and M. Scolnick, Trapping of $\mu$ Mesons in Magnet Structures, Lawrence Berkeley Laboratory Report UCRL-10143 (1965).

55. H. E. Babbitt, J. J. Doland, and J. L. Cleasby, Water Supply Engineering, Sixth Ed., McGraw-Hill, NY (1962), Sect. 2.8.

56. Edward E. Johnson, Inc., Ground Water and Wells, 4th printing, Johnson Division, UOP Inc., St. Paul, Minnesota (1975), p. 104.

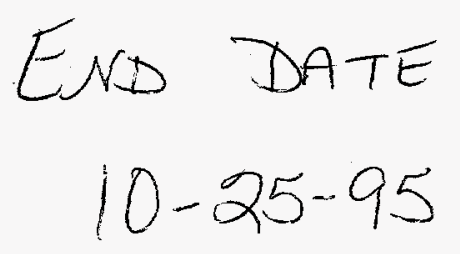

